\title{
A computational and functional study elicits the ameliorating effect of the Chinese herbal formula Huo Luo Xiao Ling Dan on experimental ischemia- induced myocardial injury in rats via inhibition of apoptosis
}

This article was published in the following Dove Press journal:

Drug Design, Development and Therapy

18 February 2015

Number of times this article has been viewed

Xiang-Dong Han,'

Zhi-Wei Zhou, ${ }^{2-4}$ Wei Yang,'

Hang-Cheng Ye, 'Ying-Zi Xu,'

Yun-Feng Huang,' Tong Zhang,'

Shu-Feng Zhou ${ }^{2}$

'School of Pharmacy, Shanghai University of Traditional Chinese Medicine, Shanghai, People's Republic of China; ${ }^{2}$ Department of Pharmaceutical Sciences, College of Pharmacy, University of South Florida, Tampa, FL, USA; ${ }^{3}$ Guizhou Provincial Key Laboratory for Regenerative Medicine, ${ }^{4}$ Stem Cell and Tissue Engineering Research Center and Sino-US Joint Laboratory for Medical Sciences, Guizhou Medical University, Guiyang, People's Republic of China

Correspondence: Xiang-Dong Han School of Pharmacy, Shanghai University of Traditional Chinese Medicine, Shanghai, People's Republic of China

Tel +86 2I 51322186

Fax +86 21 5132 2196

Email hanxd555@।63.com

Shu-Feng Zhou

Department of Pharmaceutical Sciences, College of Pharmacy, University of South Florida, I290I Bruce B. Downs Boulevard, Tampa, FL 336/2, USA

Tel +I 8139746276

Fax + I 8139059885

Email szhou@health.usf.edu
Abstract: Ischemic heart disease (IHD) is the leading cause of death worldwide and remains a major life-threatening factor in humans. Apoptosis has been implicated in the pathogenesis of IHD. The Chinese herbal formula Huo Luo Xiao Ling Dan (HLXLD), one of the commonly used Chinese herbal formulas, consists of Salviae miltiorrhizae, Angelica sinensis, Gummi olibanum, and Commiphora myrrha, with a wide spectrum of pharmacological activity. However, the mechanism of action and molecular targets of HLXLD in the treatment of IHD are unclear. This study aimed to computationally predict the molecular interactions between the major active components of HLXLD and key regulators of apoptosis and then examine the effect of HLXLD on coronary artery ligation-induced acute myocardial ischemia in rats. The molecular interactions between the major active components of HLXLD, including ferulic acid, ligustilide, succinic acid, vanillic acid, tanshinone IIA, tanshinone IIB, danshensu, salvianolic acid $A$, salvianolic acid $C$, protocatechuic aldehyde, and $\beta$-boswellic acid and human protein molecules including B cell lymphoma-extra large (Bcl-xl), B cell lymphoma 2 antagonist/killer 1 (Bak1), B cell lymphoma 2 (Bcl-2), procaspase 3, and caspase 9 with regard to hydrogen bond formation, charge interaction, and $\pi$ - $\pi$ stacking using Discovery Studio ${ }^{\circledR}$ program 3.1 . The 12 HLXLD components were predicted by ADMET (absorption, distribution, metabolism, excretion and toxicity) Predictor to have favorable pharmacokinetic and low hepatotoxicity profiles. The acute myocardial ischemia was established by surgical ligation of the left anterior descending coronary artery. The rats were divided into a sham operative group, a model group, a positive control group treated with $0.2 \mathrm{mg} / \mathrm{kg}$ isosorbide mononitrate, and groups treated with $2.7,5.4$, or $10.8 \mathrm{~g} / \mathrm{kg}$ HLXLD. The results showed that administration of HLXLD increased mean arterial pressure, left ventricular systolic pressure, heart rate, and maximal rate of rise/descent of left ventricular pressure levels. Administration of HLXLD significantly ameliorated coronary artery ligation-induced tissue damage in the left ventricle, with restored arrangement of myocardial fibers and recovered myoplasm in rats. Furthermore, HLXLD markedly increased the expression level of Bcl-2 but decreased the level of cleaved caspase 3. Taken together, administration of HLXLD attenuated acute myocardial ischemia-induced damage in cardiomyocytes and inhibited apoptotic death of cardiomyocytes, thereby exerting a cardioprotective effect in rats with IHD. These findings suggest that HLXLD may represent a promising herbal formula for the treatment of cardiovascular disease by counteracting apoptotic cell death via multiple active compounds. More studies are warranted to fully elucidate the mechanisms of action, identify the therapeutic targets, and validate the efficacy and safety of HLXLD in the treatment of IHD.

Keywords: Huo Luo Xiao Ling Dan, myocardial ischemia, coronary artery ligation, hemodynamics, apoptosis, molecular docking 


\section{Introduction}

Ischemic heart disease (IHD, also known as coronary heart disease) is the most common type of cardiovascular disease, is the leading cause of death worldwide, and places a huge burden on the individual, family, and community. ${ }^{1-3}$ There were 7,249,000 deaths resulting from IHD worldwide in 2008 , accounting for $12.7 \%$ of total global mortality. ${ }^{1}$ Worldwide, there is over 20 -fold variation in IHD mortality rates between countries. ${ }^{4}$ In the USA, IHD is the leading cause of mortality, resulting in over 500,000 deaths every year. ${ }^{1,4}$ In the People's Republic of China, IHD is the second leading cause of cardiovascular disease-related death, accounting for $22 \%$ of cardiovascular deaths in urban areas and $13 \%$ in rural areas. ${ }^{5}$ In the past few decades, although there has been great improvement in our understanding of the etiology of IHD and advances in the diagnostics and therapeutics for IHD, the disease remains a major clinical challenge with lifethreatening outcomes. Therefore, there is an urgent need for new agents and novel therapeutic approaches in the treatment of IHD to reduce the mortality and improve quality of life for patients with IHD.

Accumulating evidence shows that apoptosis is an important contributing factor in the development and progression of IHD. ${ }^{6,7}$ The balance between proapoptotic and antiapoptotic proteins is critical to maintain the cellular status of cardiomyocytes. Apoptosis can augment ischemic conditions. The B-cell lymphoma 2 ( $\mathrm{Bcl}-2)$ protein family is a determining factor in the commitment of cells to apoptosis. ${ }^{8}$ Manipulation of apoptosis by regulating the Bcl-2 family has been proposed to be a promising strategy for the treatment of many diseases, including IHD and cancer. ${ }^{8}$ Furthermore, the caspase family, aspartate-specific cysteine proteases, and members of the interleukin-1 $\beta$-converting enzyme family, are closely associated with apoptosis and have an important role in the induction, transduction, and amplification of intracellular apoptotic signals. ${ }^{9}{ }^{10}$ Caspases can be regulated by Bcl-2 family proteins, calpain, and calcium ions. ${ }^{9}{ }^{10}$ It has been reported that targeting caspase 3 may represent an attractive approach to attenuate or reverse heart failure. ${ }^{9,10}$ However, the therapeutic effect and safety of the abovementioned approaches in the treatment of cardiovascular diseases need to be confirmed.

There is increasing evidence showing that natural products have substantial therapeutic effects on cardiovascular diseases, including IHD. Huo Luo Xiao Ling Dan (HLXLD) is a classic prescription that has been documented in "Records of Tradition Chinese and Western Medicine in Combination" since the Qing Dynasty. ${ }^{11}$ HLXLD consists of Radix Salvia miltiorrhiza,
Angelica sinensis, Gummi olibanum, and Commiphora myrrha. It has a wide range of pharmacological activity, ${ }^{12-17}$ such as removing blood stasis, relieving cold pain in the heart and abdomen, ameliorating back pain or lumbago, sprains, and bruises, improving internal and external sores and ulcers, and decreasing lump accumulation in the abdomen. In recent years, increasing evidence suggests that HLXLD may be effective for the treatment of IHD, such as coronary atherosclerotic cardiopathy, due to the numerous pharmacologically active components, such as ferulic acid, ligustilide, succinic acid, vanillic acid, tanshinones, danshensu, salvianolic acids, protocatechuic aldehyde, and $\beta$-boswellic acid. ${ }^{18,19}$ However, mechanistic and functional studies of the therapeutic effects and targets of HLXLD in IHD are still sparse. In particular, there is a lack of investigation of the molecular interactions between the major active components of HLXLD and apoptotic regulators and the regulating effect of HLXLD on apoptosis in IHD. In the present study, we aimed to predict the molecular interactions between the major active components of HLXLD and apoptotic regulators and to examine the therapeutic effect of HLXLD in a rat model of acute myocardial ischemia induced by coronary artery ligation.

\section{Materials and methods Molecular docking}

We first predicted the molecular interactions between the major active components of HLXLD (ferulic acid, ligustilide, succinic acid, vanillic acid, tanshinone IIA, tanshinone IIB, danshensu, salvianolic acid A, salvianolic acid B, salvianolic acid $C$, protocatechuic aldehyde, and $\beta$-boswellic acid) and the key regulators involved in apoptotic cell death using the Discovery Studio ${ }^{\circledR}$ program 3.1 designed by Accelrys Inc (San Diego, CA, USA). The two-dimensional structure of the 12 components was retrieved from the PubChem database (Figure 1) and the three-dimensional crystal protein structures were obtained from the Research Collaboratory for Structural Bioinformatics Protein Data Bank (PDB; http://www.rcsb.org/pdb/). There were five key regulators of apoptosis that were selected for the molecular docking experiments, including human Bcl-xl (PDB ID: 4TUH), Bak1 (PDB ID: 2JCN), Bcl-2 (PDB ID: 2O21), procaspase 3 (PDB ID: 4JRO), and caspase 9 (PDB ID: 2AR9). The molecular docking experiments were performed as previously described, with some modifications. ${ }^{20,21}$ Briefly, the protein and ligand molecules were prepared prior to docking. For protein preparation, $\mathrm{Bcl}-\mathrm{xl}, \mathrm{Bak} 1, \mathrm{Bcl}-2$, procaspase 3 , and caspase 9 were cleaned, modified, and prepared for defining and editing the binding site. During preparation for ferulic 


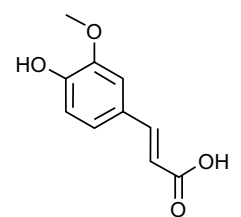

Ferulic acid<smiles>Cc1ccc2c(c1CCCO)-c1occ(C)c1C(=O)C2=O</smiles>

Tanshinone IIB<smiles>CCC/C=C1\OC(=O)C2=C1CCC=C2</smiles>

Ligustilide<smiles>O=C(O)CCC(=O)O</smiles>

Succinic acid<smiles>COc1cc(C(=O)O)ccc1O</smiles>

Vanillic acid<smiles>Cc1coc2c1C(=O)C(=O)c1c-2ccc2c1CCCC2(C)C</smiles>

Tanshinone IIA

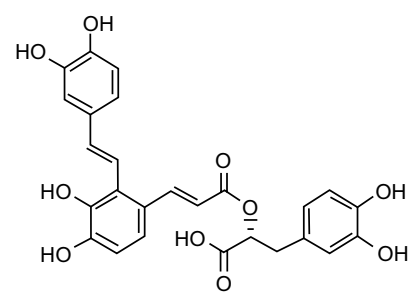

Salvianolic acid $\mathrm{A}$<smiles>O=C(O)C(O)Cc1ccc(O)c(O)c1</smiles>

Danshensu<smiles>O=Cc1ccc(O)c(O)c1</smiles>

Protocatechuic aldehyde

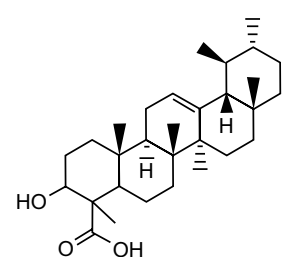

$\beta$-Boswellic acid

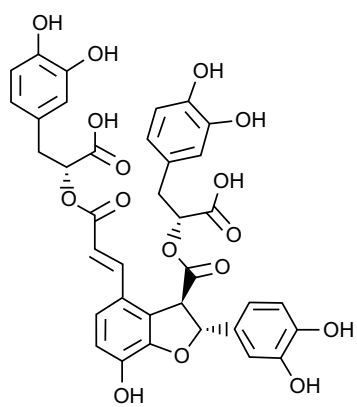

Salvianolic acid B

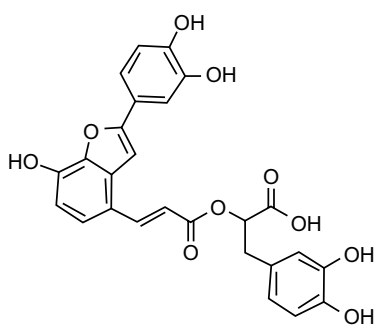

Salvianolic acid C

Figure I Chemical structures of the major active components of Huo Luo Xiao Ling Dan, including ferulic acid, ligustilide, succinic acid, vanillic acid, tanshinone IIA tanshinone IIB, danshensu, salvianolic acid A, salvianolic acid B, salvianolic acid C, protocatechuic aldehyde, and $\beta$-boswellic acid.

acid, ligustilide, succinic acid, vanillic acid, tanshinone IIA, tanshinone IIB, danshensu, salvianolic acid A, salvianolic acid $B$, salvianolic acid $C$, protocatechuic aldehyde, and $\beta$-boswellic acid, the duplicate structures were deleted, and ionization change, tautomer or isomer generation, Lipinski filter, and three-dimensional generator were all set true. A harmonic potential with a force constant of $300 \mathrm{kcal} / \mathrm{mol}$ was applied outside the grid boundary. Following preparation of the proteins, ligands, and grid setting, the 12 components were docked into the binding sites of Bcl-xl, Bak1, Bcl-2, procaspase 3, and caspase 9. Electrostatic energy and van der Waals forces were considered during the docking process. For each defined van der Waals force or electrostatic probe, the interactions with all protein atoms were stored at predetermined grid points. For ligand atoms located between grid points, a trilinear interpolation was used to approximate the energies.

\section{Prediction of ADMET of the major active components of HLXLD}

Following the prediction of the molecular interactions, we also computationally evaluated the absorption, distribution, metabolism, excretion and toxicity (ADMET) of ferulic acid, ligustilide, succinic acid, vanillic acid, tanshinone IIA, tanshinone IIB, danshensu, salvianolic acid A, salvianolic acid B, salvianolic acid $C$, protocatechuic aldehyde, and $\beta$-boswellic acid using the Discovery Studio ${ }^{\circledR}$ program 3.1 designed by Accelrys Inc as previously mentioned. ${ }^{21}$ Briefly, the chemical structures of the 12 major active components of HLXLD were retrieved from the PubChem database (Figure 1). All the structures were introduced into the Discovery Studio ${ }^{\circledR}$ program 3.1 and prepared. During the process of structure preparation, duplicate chemical structures were deleted, and ionization change, tautomer or isomer generation, Lipinski filter, and three-dimensional generator were all set true. Following the structure preparation, ferulic acid, ligustilide, succinic acid, vanillic acid, tanshinone IIA, tanshinone IIB, danshensu, salvianolic acid A, salvianolic acid B, salvianolic acid $\mathrm{C}$, protocatechuic aldehyde, and $\beta$-boswellic acid were subject to ADMET evaluation using the ADMET Predictor module. The solubility, absorption, permeability across the blood-brain barrier, interactions with cytochrome P450 (CYP)2D6, hepatotoxicity, and plasma protein binding were predicted. There are different levels for description 
of ADMET and their rules. Absorption levels ranged from $0,1,2$, to 3 , indicating good, moderate, low, or very low absorption, respectively. The predicted aqueous solubility level was classified into extremely low (0), no, very low, but possible (1), yes, low (2), yes, good (3), yes, optimal (4), no, too soluble (5), and warning, molecules with one or more unknown AlogP98 types (6). The predicted blood-brain barrier permeability levels included very high (0), high (1), medium (2), low (3), undefined (4), and warning, molecules with one or more unknown AlogP calculation (5). There are two predicted classes of CYP2D6 ligand, ie, noninhibitor (0) and inhibitor (1). The predicted hepatotoxicity was categorized into nontoxic (0) and toxic (1) effects. The predicted plasma protein binding levels included binding $<90 \%(0)$, binding $\geq 90 \%$ (1), and binding $\geq 95 \%$ (2).

\section{Chemicals and reagents}

HLXLD extract (batch no.: 2010001) was prepared in the Pharmaceutical Laboratory at Shanghai University of Traditional Chinese Medicine, Shanghai, People's Republic of China. Xinya Danxiao tablets were purchased from Shanghai New Asia Pharmaceutical Minhang Inc. (Shanghai, People's Republic of China). Urethane was obtained from Sinopharm Chemical Reagent Inc. (Shanghai, People's Republic of China). Penicillin sodium injections were sourced from Shanghai Asia Pioneer Pharmaceutical Inc. (Shanghai, People's Republic of China). Normal (0.9\%) saline was purchased from Shanghai Changzheng Fumin Jinshan Pharmaceutical Inc. (Shanghai, People's Republic of China). Superoxide dismutase (SOD), creatine kinase (CK), and lactate dehydrogenase (LDH) assay kits were purchased from Nanjing Jiancheng Technology Inc. (Nanjing, People's Republic of China). A Pierce ${ }^{\mathrm{TM}}$ bicinchoninic acid protein assay kit, skim milk, RIPA buffer, and Pierce enhanced chemiluminescence Western blotting substrate were obtained from Thermo Scientific Inc. (Hudson, NH, USA). Primary antibodies against rat caspase 3 and glyceraldehyde-3-phosphate dehydrogenase (GAPDH) were sourced from Cell Signaling Technology Inc. (Beverly, MA, USA) and the primary antibody to Bcl-2 was from Biolegend Inc. (San Diego, CA, USA). The IRDye ${ }^{\circledR}$ 680RD secondary antibody donkey anti-mouse immunoglobulin $\mathrm{G}$ was obtained from Li-Cor Biosciences Inc. (Lincoln, NE, USA).

\section{Herbal preparation}

A. sinensis, G. olibanum, and C. myrrha (500 g each) were mixed and dissolved in distilled water (1:10, w/v). A 9-mL aliquot of volatile oil was extracted from the mixture. Next, $240 \mathrm{~g}$ of powder was obtained from the aqueous solution of
Chinese angelica, frankincense, and myrrh mixture. Salvia miltiorrhiza (500 g) was dissolved in alcohol (1:10, w/v) and heated under reflux for 1 hour, and $500 \mathrm{~mL}$ of alcoholic extraction was collected by centrifuging at $3,000 \times g$ for 30 minutes. The residues were dissolved in water $(1: 10, \mathrm{w} / \mathrm{v})$. The water solution was heated under reflux for 1 hour twice, and the resulting liquids were combined with the previous extractions. The mixture was then concentrated by centrifugation to $250 \mathrm{~mL}$. The extracts of the drugs were mixed into a suspension and diluted with distilled water to prepare the low-dose $(2.7 \mathrm{~g} / \mathrm{kg})$, mid-dose $(5.4 \mathrm{~g} / \mathrm{kg})$, and high-dose $(10.8 \mathrm{~g} / \mathrm{kg})$ test.

\section{Animals}

Male Sprague-Dawley rats (200-250 g, aged 6-8 weeks) were provided by the Experimental Animal Center of Shanghai University of Traditional Chinese Medicine. The rats were housed in the accredited animal housing facility under controlled temperature $\left(22^{\circ} \mathrm{C} \pm 2^{\circ} \mathrm{C}\right)$ and a 12 -hour dark/ light cycle. All rats were allowed to acclimate for 5-7 days prior to the experiments. All experiments were conducted according to the animal protocol approved by the animal ethics committee of Shanghai University of Traditional Chinese Medicine.

\section{Animal experiment}

In order to examine the effect of HLXLD on IHD in rats, we first performed coronary artery ligation to establish an acute myocardial ischemic rat model using thoracotomy. Briefly, the animals were anesthetized by intraperitoneal injection of $25 \%$ urethane $(1.25 \mathrm{~g} / \mathrm{kg})$ and then placed in a dorsal position with limbs been fixed. Thoracotomy was then performed and coronary artery ligation was achieved by inserting a needle $2 \mathrm{~mm}$ below the bottom of the left auricula sinistra. The chest was quickly closed and the incision was sutured. The rats were monitored by an animal electrocardiogram machine. Establishment of the model was considered successful with one of following conditions: horizontal shifts of the ST-segment downward or upward by $>0.1 \mathrm{mV}$; T-wave elevation over $1 / 2$ of the $\mathrm{R}$ wave in the same lead; $\mathrm{T}$-wave elevation accompanied by ST-segment shifts; oblique or horizontal shifts of ST-segment $<0.1 \mathrm{mV}$; and a flat low pattern or two-way inversion of the T-wave. The established animal models were randomly divided into five groups $(n=8)$, including a model group, a positive control group (isosorbide mononitrate tablets), and groups treated with HLXLD 2.7, 5.4 , or $10.8 \mathrm{~g} / \mathrm{kg}$. Additionally, a sham operation group of rats underwent thoracotomy with insertion of a line but no 
coronary artery ligation. After surgery, each animal was administered sodium penicillin (40,000 U/day) by intramuscular injection for 3 consecutive days to prevent postoperative infection. At 24 hours post surgery, all experimental groups were given the test drug by intragastric administration once a day for 4 weeks. The model control group and sham operation group were given the same volume of distilled water by intragastric administration. After 4 weeks, the animals were fasted for 12 hours and then anesthetized. The left and right carotid arteries were separated and a PE-50 polyethylene catheter containing 50 units/mL heparin sodium solution was inserted. The animals were connected to a physiological recorder via a pressure transducer for recording the changes in electrocardiography and pressure. Hemodynamic parameters, including heart rate, mean arterial pressure (MAP), left ventricular systolic pressure (LVSP), left ventricular end-diastolic pressure (LVEDP), and maximum rate of left ventricular pressure rise and fall $\left( \pm \mathrm{dp} / \mathrm{dt}_{\max }\right)$ were measured and recorded. Thereafter, blood specimens were collected from the carotid artery. Serum was separated by centrifugation at $300 \times g$ and then used for CK, LDH, and SOD assays. Additionally, tissue specimens were taken from the left ventricle and divided into two parts for Western blotting assay and morphologic examination.

\section{Measurement of selected enzymes in rat plasma}

The blood samples were collected from the carotid artery and separated by centrifugation at $300 \times \mathrm{g}$. The effect of HLXLD in rats with coronary artery ligation-induced acute myocardial ischemia was evaluated by examining plasma levels of SOD, CK, and LDH. Assay kits for SOD, CK, and LDH were used to measure their levels in blood according to the manufacturer's instructions.

\section{Histopathological examination}

To examine the effect of HLXLD on the morphology of cardiomyocytes, left ventricle tissue samples were collected for routine histopathological examination as previously described. ${ }^{22}$ The tissue samples were fixed with formalin, embedded in paraffin, and sectioned. Sections $4 \mu \mathrm{m}$ thick were stained with hematoxylin and eosin and observed under light microscopy (Nikon Eclipse 50i ${ }^{\mathrm{TM}}$; Kanagawa, Japan). Magnification was $40 \times$.

\section{Western blotting assay}

The expression levels of rat heart caspase 3 and Bcl-2 were determined using the Western blotting assay as described previously. ${ }^{23}$ Samples of rat left ventricle tissue (5 mg each) were washed with pre-cold phosphate-buffered saline and homogenated in $200 \mu \mathrm{L}$ of RIPA buffer containing a protease inhibitor cocktail. The supernatant was collected twice via centrifugation at $15,000 \times g$ for 15 minutes at $4{ }^{\circ} \mathrm{C}$. The protein concentration were measured by a bicinchoninic acid protein assay kit. An equal amount of protein sample $(20 \mu \mathrm{g})$ was electrophoresed on $12 \%$ sodium dodecyl sulfate polyacrylamide gel electrophoresis (SDS-PAGE) mini-gel after thermal denaturation for 5 minutes at $95^{\circ} \mathrm{C}$. The proteins were transferred onto a methanol-activated polyvinylidene difluoride membrane at $100 \mathrm{~V}$ for 2 hours at $4^{\circ} \mathrm{C}$. Subsequently, the membranes were blocked with $5 \%$ skim milk and probed with primary antibodies to caspase 3 and Bcl-2 overnight at $4{ }^{\circ} \mathrm{C}$ and then incubated with the respective secondary antibody. Visualization was performed using an infrared fluorescence scanning and imaging system (Li-Cor Biosciences Inc., Lincoln, NE, USA). The protein level was normalized to the matching densitometric value of GAPDH.

\section{Statistical analysis}

The results are expressed as the mean \pm standard deviation. Comparison of means between groups was performed using one-way analysis of variance (ANOVA) followed by Tukey's multiple comparison. A value of $P<0.05$ was considered to be statistically significant.

\section{Results}

\section{HLXLD components interact with human $\mathrm{Bcl}-\mathrm{xl}$, Bakl, Bcl-2, procaspase 3, and caspase 9}

Following the prediction and validation of the molecular targets, we examined the possible interactions between ferulic acid, ligustilide, succinic acid, vanillic acid, tanshinone IIA, tanshinone IIB, danshensu, salvianolic acid A, salvianolic acid $\mathrm{B}$, salvianolic acid $\mathrm{C}$, protocatechuic aldehyde, and $\beta$-boswellic acid with human Bcl-xl, Bak1, Bcl-2, procaspase 3 , and caspase 9 using the Discovery Studio ${ }^{\circledR}$ program 3.1. Ferulic acid, ligustilide, succinic acid, vanillic acid, tanshinone IIA, tanshinone IIB, danshensu, salvianolic acid A, salvianolic acid $\mathrm{C}$, protocatechuic aldehyde, and $\beta$-boswellic acid were easily docked into the active sites of human Bcl-xl, Bak1, Bcl-2, procaspase 3, or caspase 9, except for salvianolic acid $\mathrm{B}$.

As shown in Table 1, the CDOCKER interaction energy ranged from -3.43985 to $33.9346 \mathrm{kcal} / \mathrm{mol}$ between ferulic acid and human Bcl-xl, Bak1, Bcl-2, procaspase 3, and caspase 9 (Table 1 and Figure 2A-E). Ferulic acid interacted 
Table I Molecular interactions between ferulic acid, ligustilide, succinic acid, vanillic acid, tanshinone IIA, tanshinone IIB, danshensu, salvianolic acid A, salvianolic acid B, salvianolic acid C, protocatechuic aldehyde, and $\beta$-boswellic acid and human Bcl-xl, Bakl, Bcl-2, procaspase 3, and caspase 9 using Discovery Studio ${ }^{\circledR}$ program 3.I

\begin{tabular}{|c|c|c|c|c|c|c|c|}
\hline Binding target & $\begin{array}{l}\text { CDOCKER } \\
\text { interaction } \\
\text { energy } \\
(\mathrm{kcal} / \mathrm{mol})\end{array}$ & $\begin{array}{l}\text { H-bond } \\
\text { number }\end{array}$ & $\begin{array}{l}\text { Residues } \\
\text { involved in } \\
\text { H-bond } \\
\text { formation }\end{array}$ & $\begin{array}{l}\text { Charge } \\
\text { interactions }\end{array}$ & $\begin{array}{l}\text { Residues } \\
\text { involved in } \\
\text { charge } \\
\text { interactions }\end{array}$ & $\begin{array}{l}\pi-\pi \\
\text { stacking }\end{array}$ & $\begin{array}{l}\text { Residues } \\
\text { involved in } \\
\pi-\pi \text { stacking }\end{array}$ \\
\hline \multicolumn{8}{|l|}{ Ferulic acid } \\
\hline Bcl-xl (PDB ID: 4TUH) & 27.8853 & 1 & O-Leul08 & 1 & O-ArgI02 & I & Phel05 \\
\hline BakI (PDB ID: 2JCN) & 23.8894 & 0 & - & 1 & O-Glu33 & 1 & Phe40 \\
\hline Bcl-2 (PDB ID: 2O2I) & -3.43985 & 0 & - & 0 & - & 0 & - \\
\hline Procaspase 3 (PDB ID: 4JRO) & 32.8532 & 1 & O-LysI59 & 1 & O-Lys159 & 0 & - \\
\hline \multirow[t]{3}{*}{ Caspase 9 (PDB ID: 2AR9) } & 33.9346 & 2 & O-Argl 80 & 3 & O-Arg 180 & 0 & - \\
\hline & & & O-Gln285 & & O-Gln285 & & \\
\hline & & & & & O-Ser287 & & \\
\hline \multicolumn{8}{|l|}{ Ligustilide } \\
\hline Bcl-xl (PDB ID: 4TUH) & 24.0792 & 0 & - & 0 & - & 0 & - \\
\hline BakI (PDB ID: 2JCN) & 25.9178 & 0 & - & 0 & - & 1 & Phe40 \\
\hline Bcl-2 (PDB ID: 2O2I) & $-|5.974|$ & 1 & O-Phel95 & 0 & - & 0 & - \\
\hline Procaspase 3 (PDB ID: 4JRO) & 22.4759 & 1 & O-ArgI5 & 0 & - & 1 & $\operatorname{Arg} 15$ \\
\hline Caspase 9 (PDB ID: 2AR9) & 24.9135 & 0 & - & 0 & - & 0 & - \\
\hline \multicolumn{8}{|l|}{ Succinic acid } \\
\hline Bcl-xl (PDB ID: 4TUH) & 23.8093 & 0 & - & I & O-ArgI 02 & 0 & - \\
\hline BakI (PDB ID: 2JCN) & 17.7988 & 0 & - & 1 & O-Glu33 & 0 & - \\
\hline Bcl-2 (PDB ID: 2O2I) & 20.7002 & 0 & - & 0 & - & 0 & - \\
\hline \multirow[t]{2}{*}{ Procaspase 3 (PDB ID: 4JRO) } & 40.3633 & 2 & O-Asn37 & 2 & O-ArgI5 & 0 & - \\
\hline & & & O-Gly38 & & O-ArgI5 & & \\
\hline \multirow[t]{3}{*}{ Caspase 9 (PDB ID: 2AR9) } & 34.9818 & 3 & O-ArgI80 & 3 & O-ArgI 78 & 0 & - \\
\hline & & & O-G $\ln 285$ & & O-Arg 180 & & \\
\hline & & & O-Ser287 & & O-Gln285 & & \\
\hline \multicolumn{8}{|l|}{ Vanillic acid } \\
\hline Bcl-xl (PDB ID: 4TUH) & 27.1178 & 0 & - & 0 & - & 0 & - \\
\hline BakI (PDB ID: 2JCN) & 19.8383 & 1 & H-PhellI & 1 & O-Leul I8 & 0 & - \\
\hline Bcl-2 (PDB ID: 2O2I) & 16.0496 & 0 & - & 0 & - & 0 & - \\
\hline \multirow[t]{4}{*}{ Procaspase 3 (PDB ID: 4JRO) } & 29.7815 & 4 & $\mathrm{H}-\mathrm{Gly} 12$ & 1 & O-ArgI5 & 1 & O-ArgI5 \\
\hline & & & O-ArgI5 & & & & \\
\hline & & & O-Gly18 & & & & \\
\hline & & & O-Asn90 & & & & \\
\hline \multirow[t]{2}{*}{ Caspase 9 (PDB ID: 2AR9) } & 33.0769 & 1 & O-ArgI80 & 2 & O-ArgI 78 & 0 & - \\
\hline & & & & & O-Arg 180 & & \\
\hline \multicolumn{8}{|l|}{ Tanshinone IIA } \\
\hline \multirow[t]{5}{*}{ Bcl-xl (PDB ID: 4TUH) } & 10.3489 & 0 & - & 0 & - & 5 & $\operatorname{Arg} 139$ \\
\hline & & & & & & & $\operatorname{Arg} 139$ \\
\hline & & & & & & & Phel05 \\
\hline & & & & & & & Phel05 \\
\hline & & & & & & & Phel05 \\
\hline \multirow[t]{3}{*}{ BakI (PDB ID: 2JCN) } & 19.5827 & 0 & - & 0 & - & 3 & Phe40 \\
\hline & & & & & & & Phe40 \\
\hline & & & & & & & Phe40 \\
\hline Procaspase 3 (PDB ID: 4JRO) & 30.0502 & 0 & - & 0 & - & I & $\operatorname{Arg} 15$ \\
\hline Caspase 9 (PDB ID: 2AR9) & 30.1231 & 0 & - & 0 & - & 0 & - \\
\hline \multicolumn{8}{|l|}{ Tanshinone IIB } \\
\hline \multirow[t]{5}{*}{ Bcl-xl (PDB ID: 4TUH) } & 9.27765 & $\mathrm{I}$ & H-Ala I42 & 0 & - & 5 & $\operatorname{Arg} 139$ \\
\hline & & & & & & & Arg139 \\
\hline & & & & & & & Phel05 \\
\hline & & & & & & & Phel05 \\
\hline & & & & & & & Phel05 \\
\hline \multirow[t]{3}{*}{ BakI (PDB ID: 2JCN) } & 16.1665 & 0 & - & 0 & - & 3 & Phe40 \\
\hline & & & & & & & Phe40 \\
\hline & & & & & & & Phe40 \\
\hline
\end{tabular}


Table I (Continued)

\begin{tabular}{|c|c|c|c|c|c|c|c|}
\hline Binding target & $\begin{array}{l}\text { CDOCKER } \\
\text { interaction } \\
\text { energy } \\
\text { (kcal/mol) }\end{array}$ & $\begin{array}{l}\text { H-bond } \\
\text { number }\end{array}$ & $\begin{array}{l}\text { Residues } \\
\text { involved in } \\
\mathrm{H} \text {-bond } \\
\text { formation }\end{array}$ & $\begin{array}{l}\text { Charge } \\
\text { interactions }\end{array}$ & $\begin{array}{l}\text { Residues } \\
\text { involved in } \\
\text { charge } \\
\text { interactions }\end{array}$ & $\begin{array}{l}\pi-\pi \\
\text { stacking }\end{array}$ & $\begin{array}{l}\text { Residues } \\
\text { involved in } \\
\pi-\pi \text { stacking }\end{array}$ \\
\hline \multirow[t]{3}{*}{ Procaspase 3 (PDB ID: 4JRO) } & 33.0863 & 3 & O-Ilel7 & 0 & - & 3 & $\operatorname{Arg} 15$ \\
\hline & & & H-Gly92 & & & & \\
\hline & & & O-Gly92 & & & & \\
\hline Caspase 9 (PDB ID: 2AR9) & 35.7031 & 0 & - & 0 & - & 1 & Phe35I \\
\hline \multicolumn{8}{|l|}{ Danshensu } \\
\hline Bcl-xl (PDB ID: 4TUH) & 30.1811 & $\mathrm{I}$ & O-ArgI02 & 0 & - & 1 & O-Arg139 \\
\hline BakI (PDB ID: 2JCN) & 27.7588 & 1 & O-Gln66 & 0 & - & 1 & Phe40 \\
\hline Bcl-2 (PDB ID: 2O2I) & 13.0233 & 0 & - & 0 & - & 1 & Ile 186 \\
\hline \multirow[t]{5}{*}{ Procaspase 3 (PDB ID: 4JRO) } & 39.3839 & 5 & $\mathrm{H}$-Serl4 & 2 & O-ArgI5 & 1 & $\operatorname{Arg} 15$ \\
\hline & & & O-Ser 14 & & O-Asn37 & & \\
\hline & & & $\mathrm{H}-\operatorname{Arg} 15$ & & & & \\
\hline & & & O-Glyl8 & & & & \\
\hline & & & O-Asn-90 & & & & \\
\hline Caspase 9 (PDB ID: 2AR9) & 34.2947 & 1 & O-Gln285 & 1 & O-ArgI 80 & 1 & $\operatorname{Arg} 180$ \\
\hline \multicolumn{8}{|l|}{ Salvianolic acid A } \\
\hline \multirow[t]{3}{*}{ Bcl-xl (PDB ID: 4TUH) } & -25.7507 & 3 & H-Glu98 & 0 & - & 3 & $\operatorname{Arg} 102$ \\
\hline & & & H-Ser 145 & & & & Phel05 \\
\hline & & & O-Serl45 & & & & $\operatorname{Arg} 132$ \\
\hline \multirow[t]{3}{*}{ Procaspase 3 (PDB ID: 4JRO) } & 53.6318 & 3 & H-Serl 4 & $\mathrm{I}$ & O-ArgI5 & 2 & $\operatorname{Arg} 15$ \\
\hline & & & O-Ser I4 & & & & Arg95 \\
\hline & & & O-Asn37 & & & & \\
\hline \multirow[t]{4}{*}{ Caspase 9 (PDB ID: 2AR9) } & -14.8647 & 4 & O-ArgI 80 & 0 & - & 2 & $\operatorname{Arg} 178$ \\
\hline & & & H-Thrl8I & & & & $\operatorname{Arg} 180$ \\
\hline & & & H-Thrl8I & & & & \\
\hline & & & O-Gln285 & & & & \\
\hline \multicolumn{8}{|l|}{ Salvianolic acid C } \\
\hline \multirow[t]{3}{*}{ Bcl-xl (PDB ID: 4TUH) } & -37.3485 & 3 & O-Argl 02 & 0 & - & 3 & Argl 02 \\
\hline & & & H-Glul29 & & & & $\operatorname{Arg} 102$ \\
\hline & & & H-GluI29 & & & & $\operatorname{Arg} 102$ \\
\hline \multirow[t]{7}{*}{ Procaspase 3 (PDB ID: 4JRO) } & 52.9465 & 7 & O-Gly18 & 2 & O-ArgI5 & 2 & $\operatorname{Arg} 15$ \\
\hline & & & O-Asn35 & & O-Gly38 & & $\operatorname{Arg} 15$ \\
\hline & & & O-Asn35 & & & & \\
\hline & & & O-Asn37 & & & & \\
\hline & & & O-Ala62 & & & & \\
\hline & & & O-Val64 & & & & \\
\hline & & & O-Asn90 & & & & \\
\hline \multirow[t]{5}{*}{ Caspase 9 (PDB ID: 2AR9) } & 32.6288 & 5 & O-ArgI 80 & I & O-Aspl 86 & 2 & $\operatorname{Arg} 178$ \\
\hline & & & O-Argl 80 & & & & $\operatorname{Arg} 180$ \\
\hline & & & O-Gln285 & & & & \\
\hline & & & O-Ser287 & & & & \\
\hline & & & O-Gly360 & & & & \\
\hline \multicolumn{8}{|l|}{ Protocatechuic aldehyde } \\
\hline \multirow[t]{2}{*}{ Bcl-xl (PDB ID: 4TUH) } & 24.6477 & 2 & O-ArgI02 & 0 & - & 0 & - \\
\hline & & & H-Serl45 & & & & \\
\hline BakI (PDB ID: 2JCN) & $|8.843|$ & 0 & - & 0 & - & 1 & Phe40 \\
\hline $\mathrm{Bcl}-2$ (PDB ID: 2O2I) & 11.8606 & 0 & - & 0 & - & 0 & - \\
\hline \multirow[t]{4}{*}{ Procaspase 3 (PDB ID: 4JRO) } & 16.6186 & 4 & H-Gly 12 & 0 & - & 1 & $\operatorname{Arg} 15$ \\
\hline & & & $\mathrm{H}-\operatorname{Arg} 15$ & & & & \\
\hline & & & O-Gly18 & & & & \\
\hline & & & O-Asn90 & & & & \\
\hline \multirow[t]{2}{*}{ Caspase 9 (PDB ID: 2AR9) } & 21.4411 & 2 & O-ArgI 80 & 0 & - & 0 & - \\
\hline & & & H-Aspl86 & & & & \\
\hline \multicolumn{8}{|l|}{$\beta$-boswellic acid } \\
\hline Procaspase 3 (PDB ID: 4JRO) & 31.6175 & $\mathrm{I}$ & O-ArgI5 & 0 & - & 0 & - \\
\hline Caspase 9 (PDB ID: 2AR9) & 7.52648 & $\mathrm{I}$ & O-ArgI 78 & 0 & - & 0 & - \\
\hline
\end{tabular}

Note: The molecular docking of salvianolic acid $B$ to all protein molecules failed. Abbreviation: PDB ID, Protein Data Bank identifier. 
A

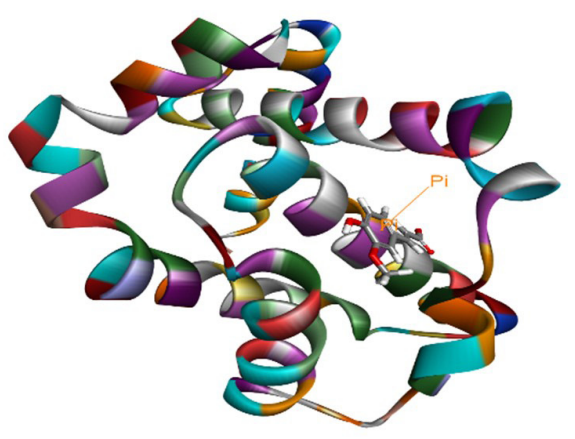

B

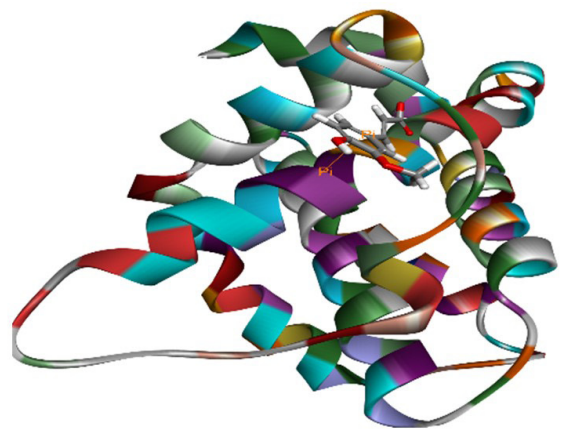

C

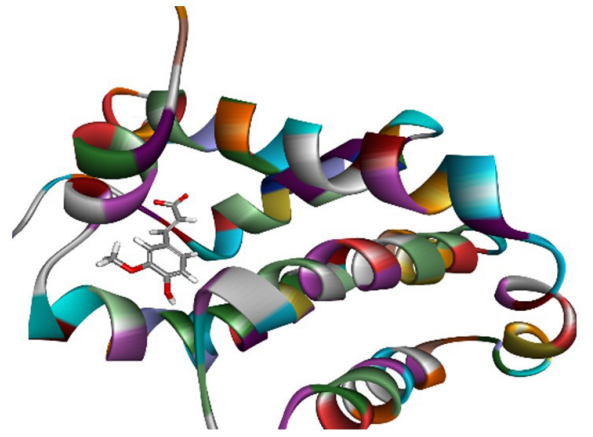

D

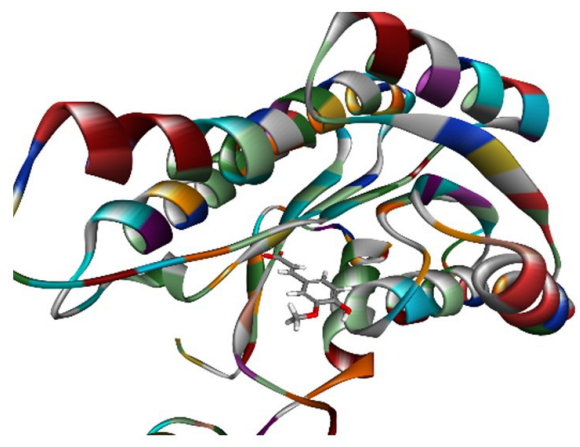

E

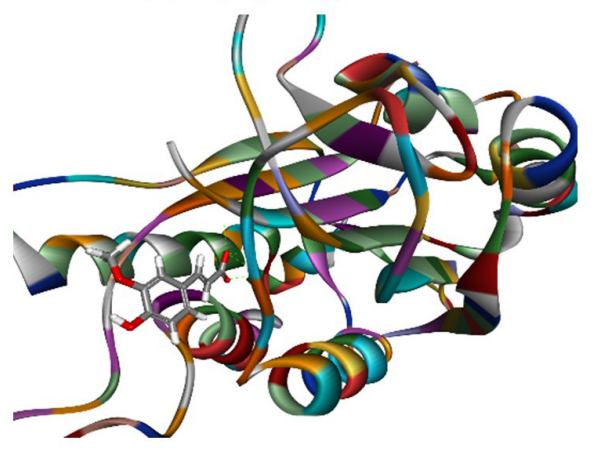

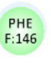<smiles>[SiH3]C1=[Si]C2CC1C2</smiles>

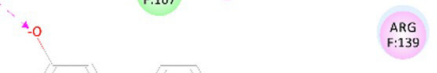

$\underset{\text { SER }}{\mathrm{A}: 37}$

(.).N

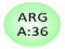

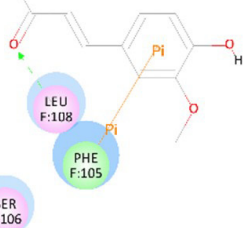

$\underset{\substack{\text { ARG } \\ \text { Fin } 139}}{ }$
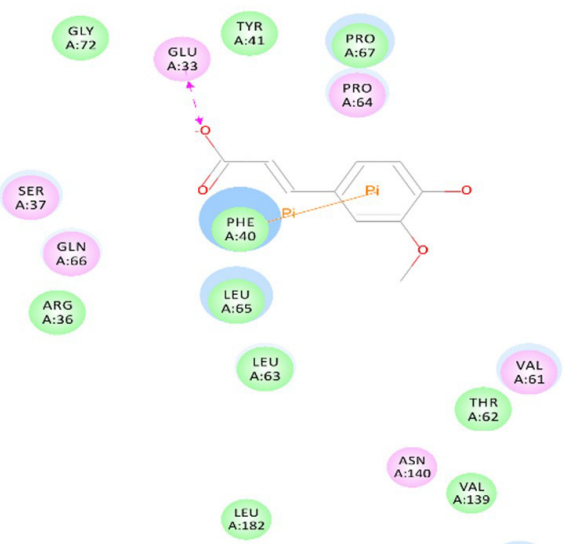

AAL

VAL
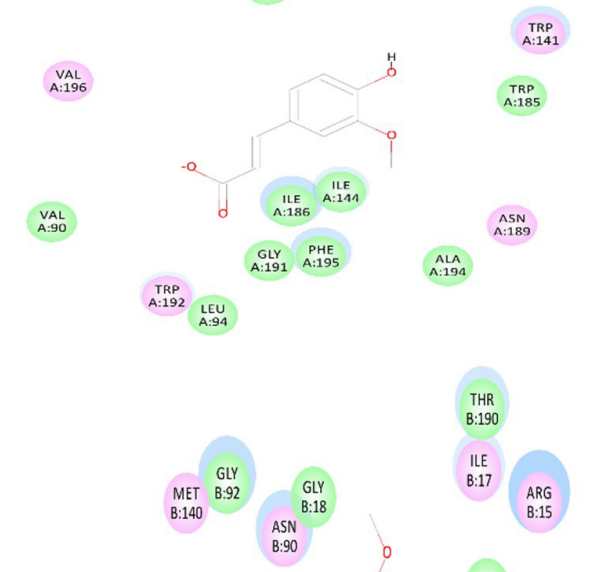

T:12
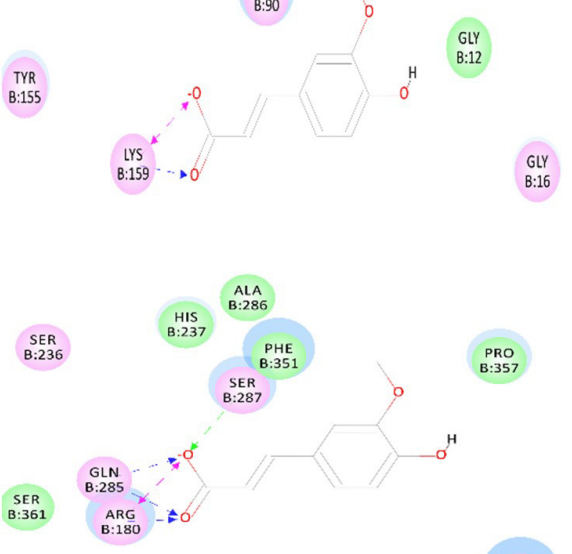

A: $A$ B

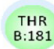

Figure 2 Molecular interactions between ferulic acid and human Bcl-xl, Bakl, Bcl-2, procaspase 3, and caspase 9 determined using Discovery Studio $($ program 3.I. Notes: (A) Bcl-xI (PDB ID: 4TUH), (B) BakI (PDB ID: 2JCN), (C) Bcl-2 (PDB ID: 2O2I), (D) procaspase 3 (PDB ID: 4JRO), and (E) caspase 9 (PDB ID: 2AR9). Abbreviation: PDB ID, Protein Data Bank identifier. 
with human Bcl-xl via hydrogen bond formation, charge interaction, and $\pi-\pi$ interaction with Leu108, $\operatorname{Arg} 102$, and Phe105 at the active site, respectively (Figure 2). There was a charge interaction and $\pi-\pi$ interaction between ferulic acid and Bak1 with Glu33 and Phe40, respectively. Ferulic acid interacted with procaspase 3 via hydrogen bond formation and charge interaction at Lys159, and ferulic acid bound to caspase 9 via hydrogen bond formation at $\operatorname{Arg} 180$ and Gln285 and charge interaction at Arg180, Gln285, and Ser287 (Figure 2). In contrast, there was no hydrogen bond formation, charge interaction, or $\pi-\pi$ interaction between ferulic acid and Bcl-2 (Figure 2).

Ligustilide was able to be docked into the active sites of human Bcl-xl, Bak1, Bcl-2, procaspase 3, and caspase 9 (Table 1 and Figure 3A-E). The CDOCKER interaction energy ranged from -15.9741 to $25.9178 \mathrm{kcal} / \mathrm{mol}$. Ligustilide bound to Bak1 and Bcl- 2 via $\pi-\pi$ interaction at Phe40 and hydrogen bond formation at Phe195, respectively (Figure 3). Ligustilide also interacted with procaspase 3 via hydrogen bond formation and $\pi-\pi$ interaction at Arg 15 (Figure 3). There was no hydrogen bond formation, charge interaction, or $\pi-\pi$ interaction between ligustilide and $\mathrm{Bcl}-\mathrm{xl}$ and caspase 9 (Figure 3).

As shown in Table 1, the CDOCKER interaction energy ranged from 17.7988 to $40.3633 \mathrm{kcal} / \mathrm{mol}$ between succinic acid and human Bcl-xl, Bak1, Bcl-2, procaspase 3, and caspase 9 (Table 1 and Figure 4A-E). Succinic acid bound to human Bcl-xl and Bak1 via charge interaction with Arg102 and Glu33 at the active sites, respectively (Figure 4). Succinic acid interacted with procaspase 3 via formation of two hydrogen bonds at Asn37 and Gly38 and two charge interactions at Arg15. Moreover, there were three hydrogen bonds formed between succinic acid and caspase 9 at O-Arg180, O-Gln285, and $\mathrm{O}-\mathrm{Ser} 287$, and there were three charge interactions between succinic acid caspase 9 at O-Arg178, O-Arg180, and O-Gln285 (Figure 4). Succinic acid was docked into the active site of Bcl-2 without hydrogen bond formation, charge interaction, or $\pi-\pi$ interaction (Figure 4 ).

Vanillic acid was docked into the active sites of human Bcl-xl, Bak1, Bcl-2, procaspase 3, and caspase 9, with the CDOCKER interaction energy ranging from 16.0496 to $33.0769 \mathrm{kcal} / \mathrm{mol}$ (Table 1 and Figure 5A-E). Vanillic acid bound to Bak1 via hydrogen bond formation at Phe111 and charge interaction at Leu118. There were four hydrogen bonds formed between vanillic acid and procaspase 3 at Gly12, Arg15, Gly18, and Asn90. Vanillic acid also interacted with procaspase 3 via charge interaction and $\pi-\pi$ stacking at Arg15 (Figure 5). There was hydrogen bond formation at Arg180 and two charge interactions at Arg178 and Arg180 between vanillic acid and caspase 9. There was no hydrogen bond formation, charge interaction, or $\pi-\pi$ interaction between vanillic acid and Bcl-xl and Bcl-2 (Figure 5).

Tanshinone IIA interacted with human Bcl-xl, Bak1, procaspase 3 , and caspase 9 mainly through $\pi-\pi$ interaction, with the CDOCKER interaction energy ranging from 10.3489 to $30.1231 \mathrm{kcal} / \mathrm{mol}$ (Table 1 and Figure $6 \mathrm{~A}-\mathrm{D})$. There were five $\pi-\pi$ interactions between tanshinone IIA and $\mathrm{Bcl}-\mathrm{xl}$ at Arg139 and Phe105 and there were three $\pi-\pi$ interactions between tanshinone IIA and Bak1 at Phe40. Tanshinone IIA also bound to procaspase 3 via $\pi-\pi$ interaction at Arg 15 . There was no hydrogen bond formation, charge interaction, or $\pi-\pi$ interaction between tanshinone IIA and Bax 1 (Figure 6). Similarly, tanshinone IIB interacted with human Bcl-xl, Bak1, procaspase 3, and caspase 9 mainly through $\pi-\pi$ interaction, and to a lesser extent via hydrogen bond formation, with CDOCKER interaction energy ranging from 9.27765 to $35.7031 \mathrm{kcal} / \mathrm{mol}$ (Table 1 and Figure 7A-D). There were five $\pi-\pi$ interactions between tanshinone IIB and Bcl-xl at Arg139 and Phe105 and three $\pi$ - $\pi$ interactions between tanshinone IIB and Bak1 at Phe40. Tanshinone IIB formed three hydrogen bonds with procaspase 3 at Ile 17 and Gly92. Moreover, tanshinone IIB bound to procaspase 3 via $\pi-\pi$ interactions at $\operatorname{Arg} 15$. There was also one $\pi-\pi$ interaction between tanshinone IIB and caspase 9 (Figure 7).

Danshensu interacted with human Bcl-xl, Bak1, Bcl-2, procaspase 3 , and caspase 9 , with CDOCKER interaction energy ranging from 13.0233 to $39.3839 \mathrm{kcal} / \mathrm{mol}$ (Table 1 and Figure 8A-E). Danshensu bound to Bcl-xl and Bak1 via hydrogen bond formation at Arg102 and Gln66, and $\pi-\pi$ stacking at Arg139 and Phe40, respectively (Figure 8). There was a $\pi-\pi$ stacking between danshensu and Bcl-2 at Ile186. Furthermore, there were five hydrogen bonds formed at Ser14, Arg15, Gly18, and Asn90, two charge interactions at $\operatorname{Arg} 15$ and Asn37, and one $\pi$ - $\pi$ stacking at Arg 15 between danshensu and procaspase 3. Moreover, danshensu bound to caspase 9 via hydrogen bond formation at Gln285, charge interaction, and $\pi-\pi$ stacking at Arg180 (Figure 8).

Salvianolic acid A was docked into the active sites of human Bcl-xl, procaspase 3, and caspase 9 (Figure 9A-C). The CDOCKER interaction energy ranged from -25.7507 to $53.6318 \mathrm{kcal} / \mathrm{mol}$ (Table 1). Salvianolic acid A bound to Bcl-xl via hydrogen bond formation at Glu98 and Ser145 and $\pi-\pi$ stacking at Arg102, Phe105, and Arg132. Salvianolic acid A also formed three hydrogen bonds with procaspase 3 at Ser14 and Asn37 and $\pi-\pi$ stacking at Arg15 and 
A

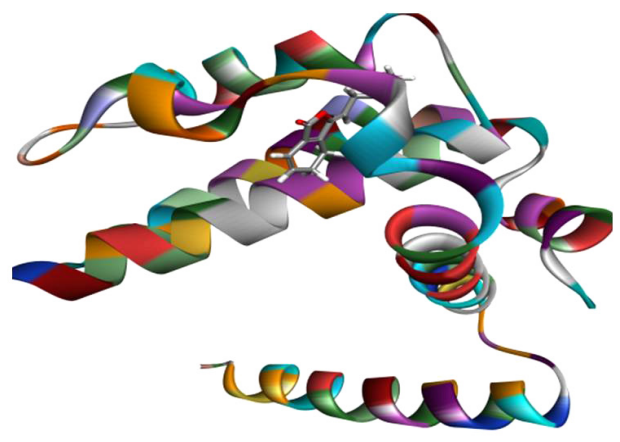

B

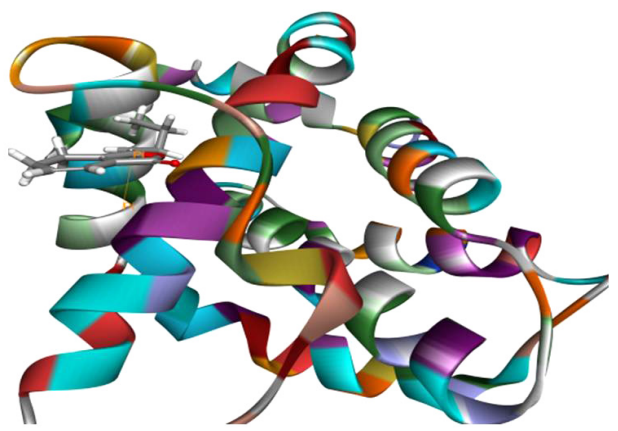

C

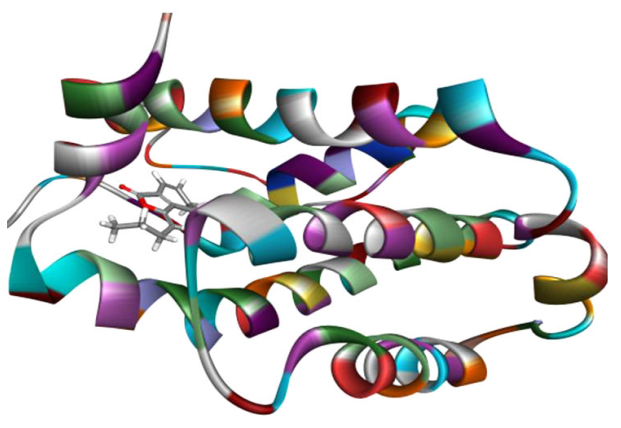

D

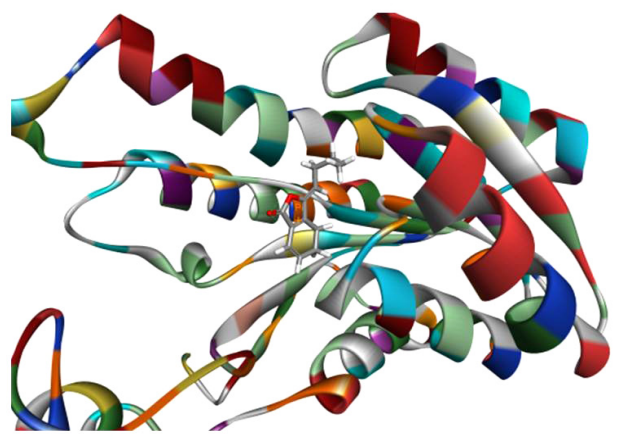

E

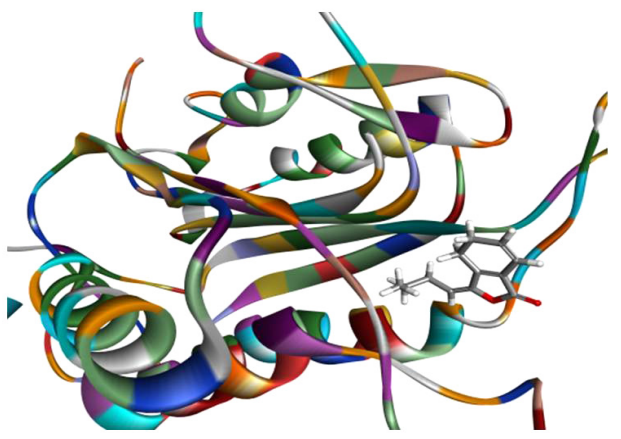

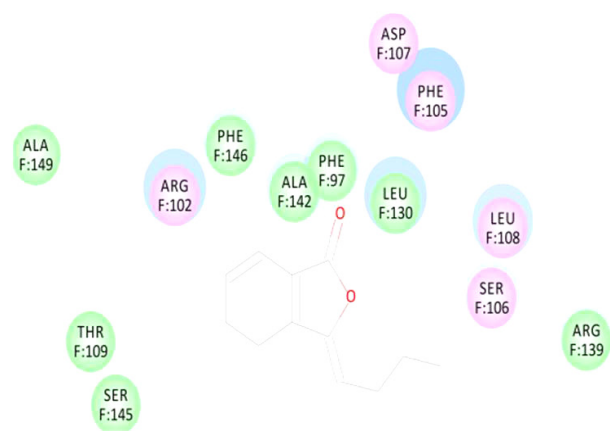

THR

LEU
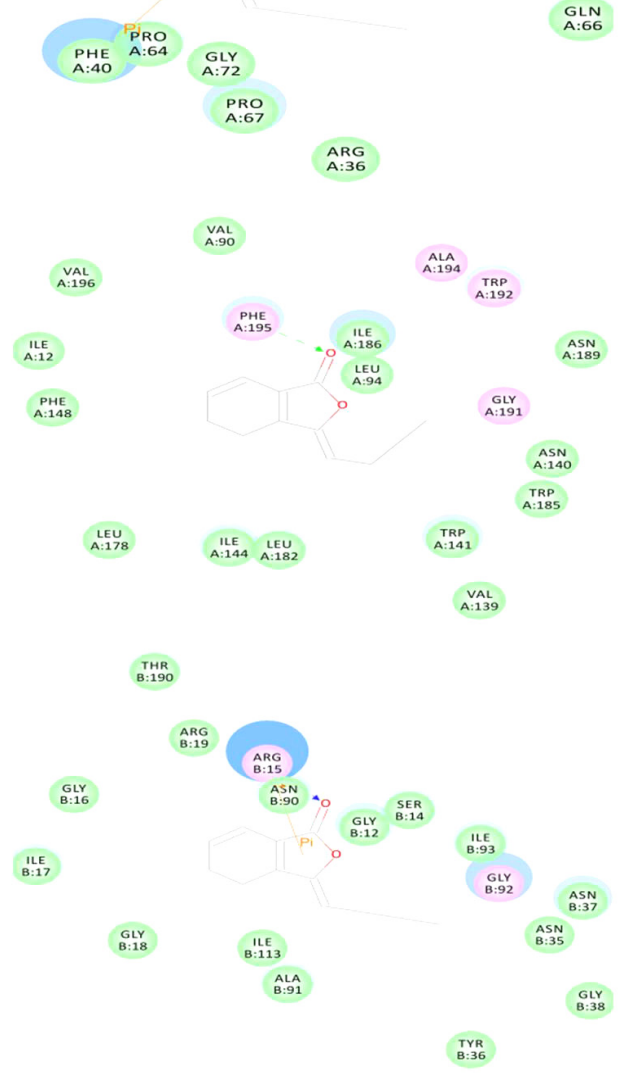

ARG

8.157

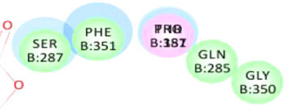

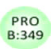

A. AR6

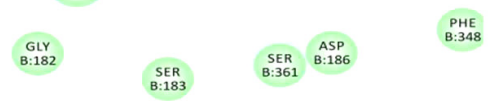

Figure 3 Molecular interactions between ligustilide and human $\mathrm{Bcl}-\mathrm{xl}$, Bakl, Bcl-2, procaspase 3, and caspase 9 determined using Discovery Studio ${ }^{\circledR}$ program 3.I. Notes: (A) Bcl-xl (PDB ID: 4TUH), (B) BakI (PDB ID: 2JCN), (C) Bcl-2 (PDB ID: 2O2I), (D) procaspase 3 (PDB ID: 4JRO), and (E) caspase 9 (PDB ID: 2AR9). Abbreviation: PDB ID, Protein Data Bank identifier. 
A

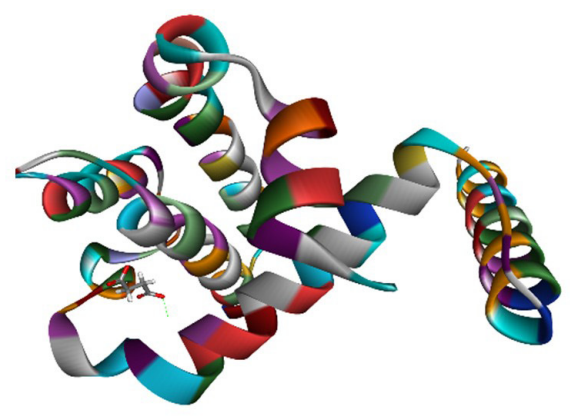

B

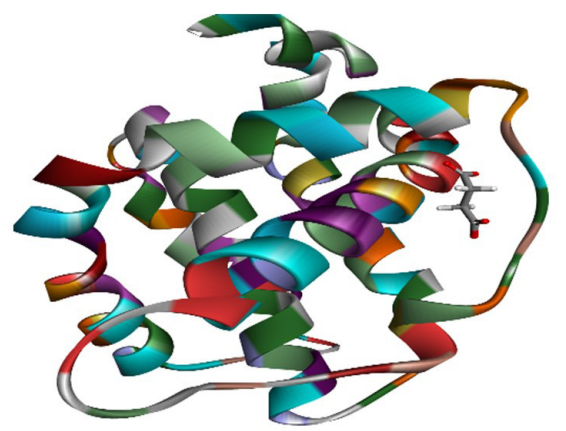

C

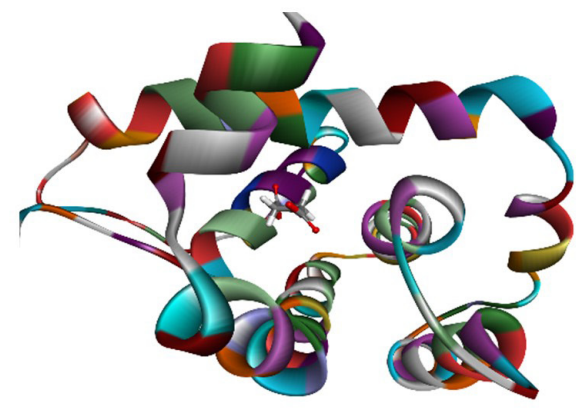

D

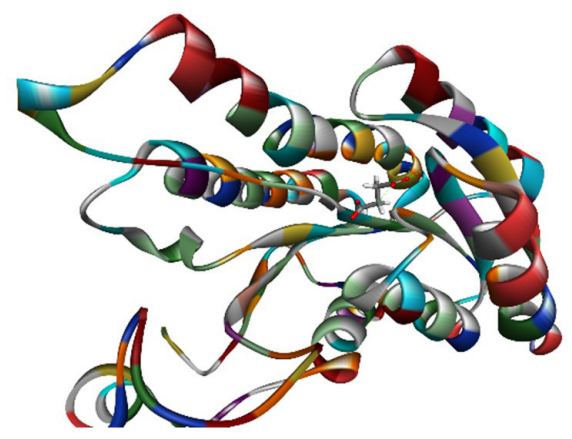

E

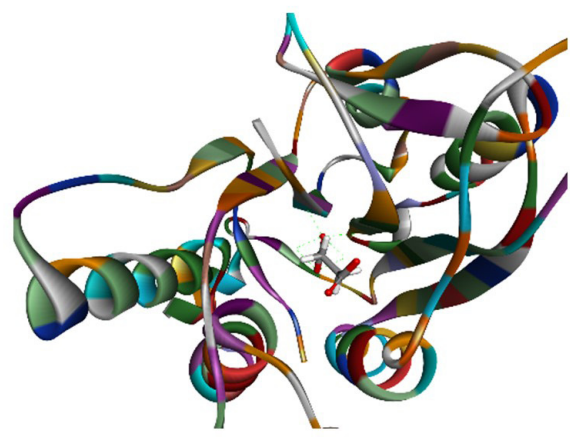

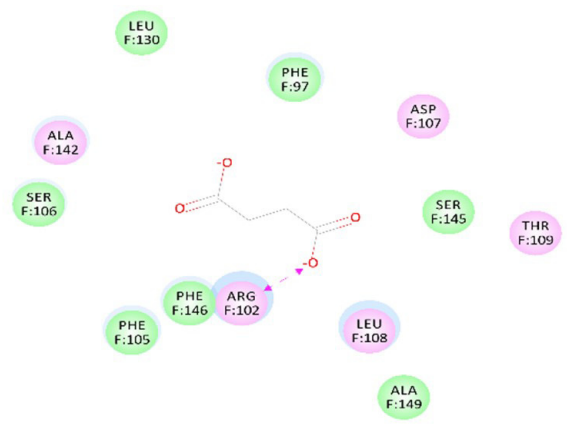
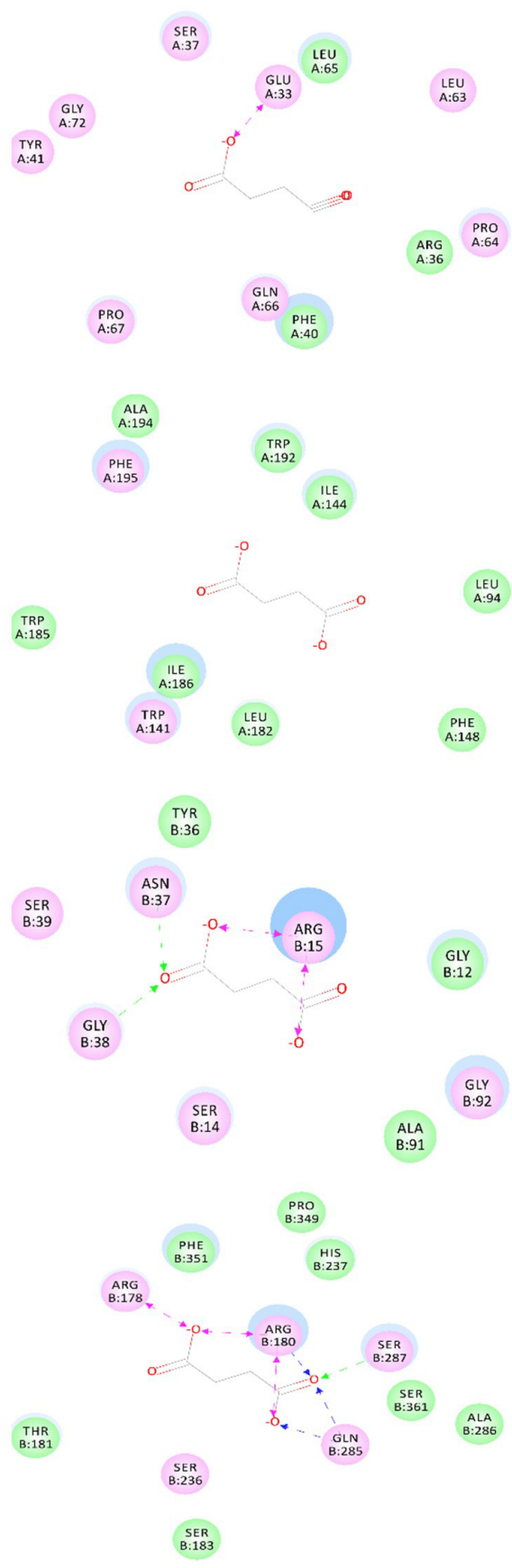

Figure 4 Molecular interactions between succinic acid and Bcl-xl, Bakl, Bcl-2, procaspase 3, and caspase 9 determined using Discovery Studio ${ }^{\circledR}$ program 3.I. Notes: (A) Bcl-xl (PDB ID: 4TUH), (B) BakI (PDB ID: 2JCN), (C) Bcl-2 (PDB ID: 2O2I), (D) procaspase 3 (PDB ID: 4JRO), and (E) caspase 9 (PDB ID: 2AR9). Abbreviation: PDB ID, Protein Data Bank identifier. 
A

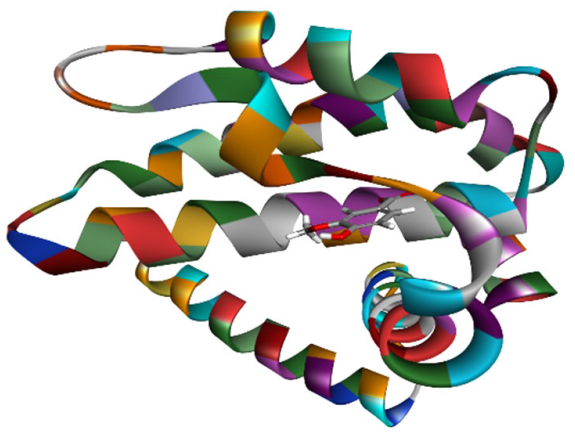

B

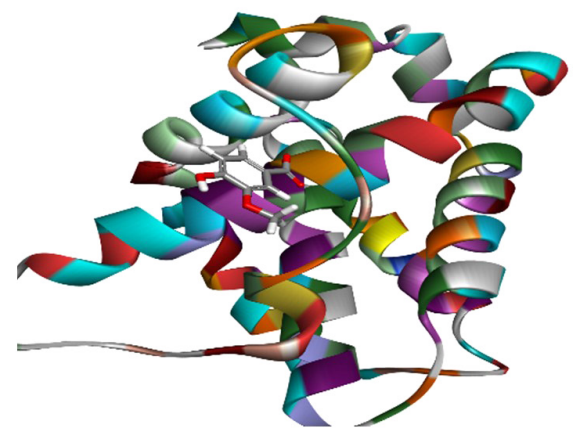

C

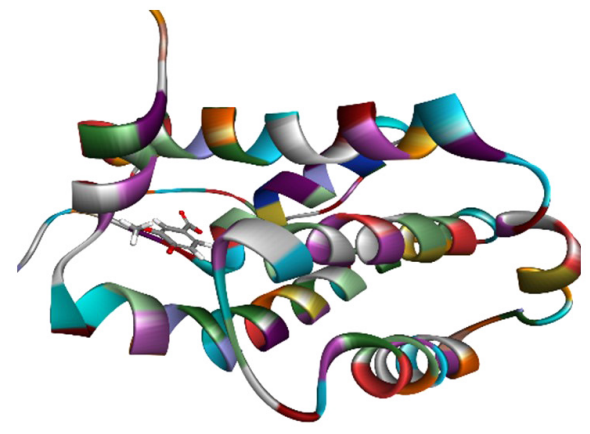

D

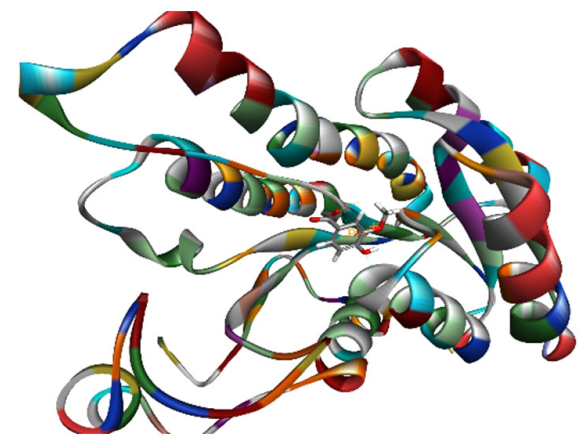

E

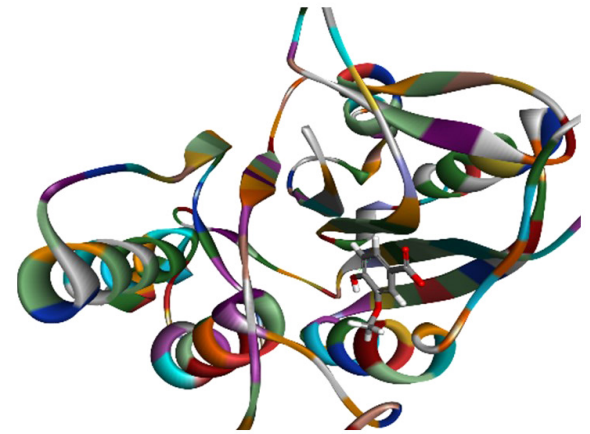

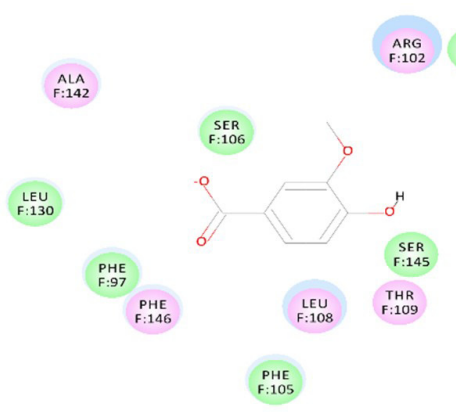

PPE

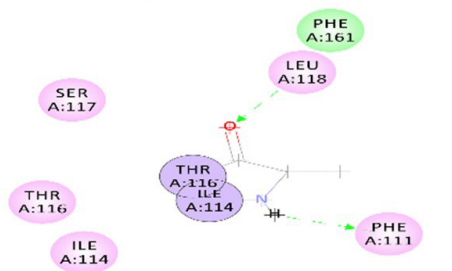

L LEU

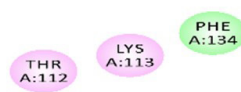

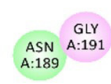

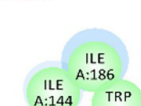

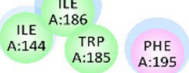

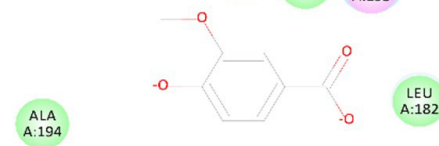

A.ta LEU

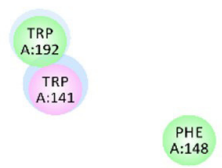

$\underset{\text { B: }}{\text { G:Y }}$

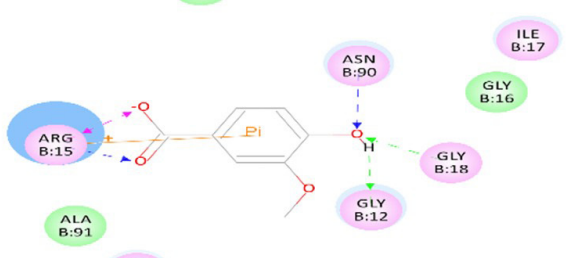

$\underset{\substack{S \in R \\ B: 14}}{B=R}$

\section{B:19 $\underset{\substack{\text { ARG } \\ \text { B:I }}}{\substack{\text { B. } \\ \text { B. }}}$}

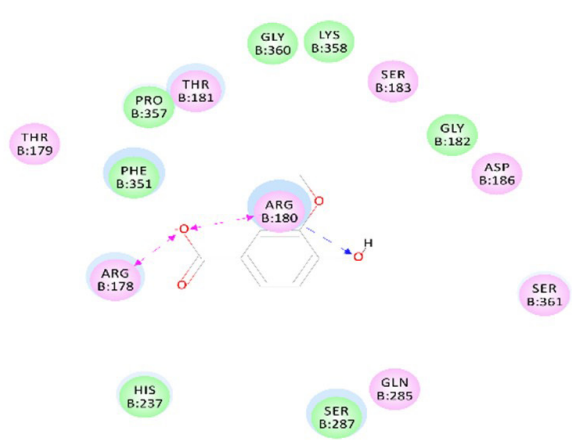

Figure 5 Molecular interactions between vanillic acid and human Bcl-xl, Bakl, Bcl-2, procaspase 3, and caspase 9 determined using Discovery Studio ${ }^{\circledR}$ program 3.I. Notes: (A) Bcl-xl (PDB ID: 4TUH), (B) BakI (PDB ID: 2JCN), (C) Bcl-2 (PDB ID: 2O2I), (D) procaspase 3 (PDB ID: 4JRO), and (E) caspase 9 (PDB ID: 2AR9). Abbreviation: PDB ID, Protein Data Bank identifier. 
A
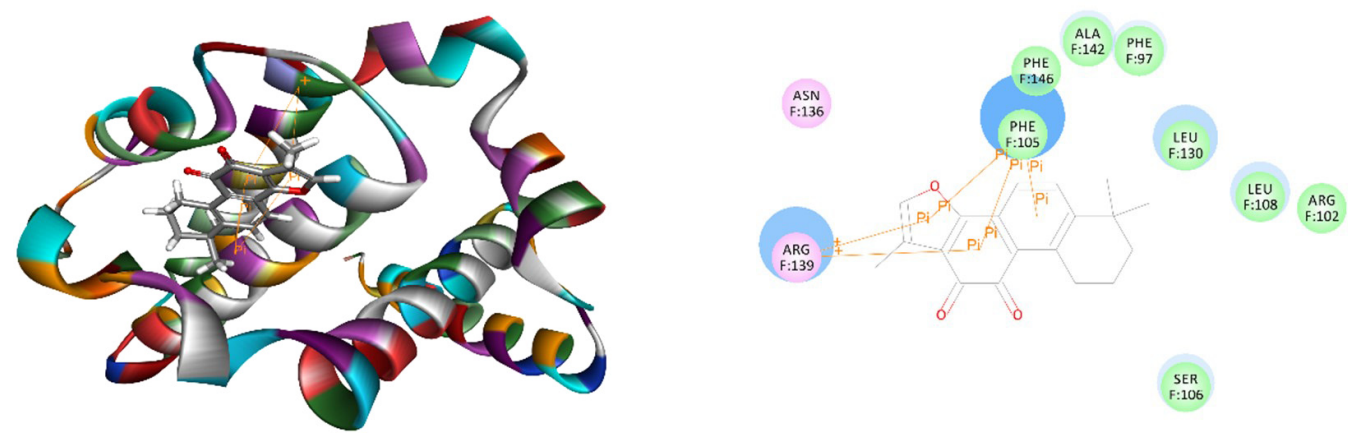

B
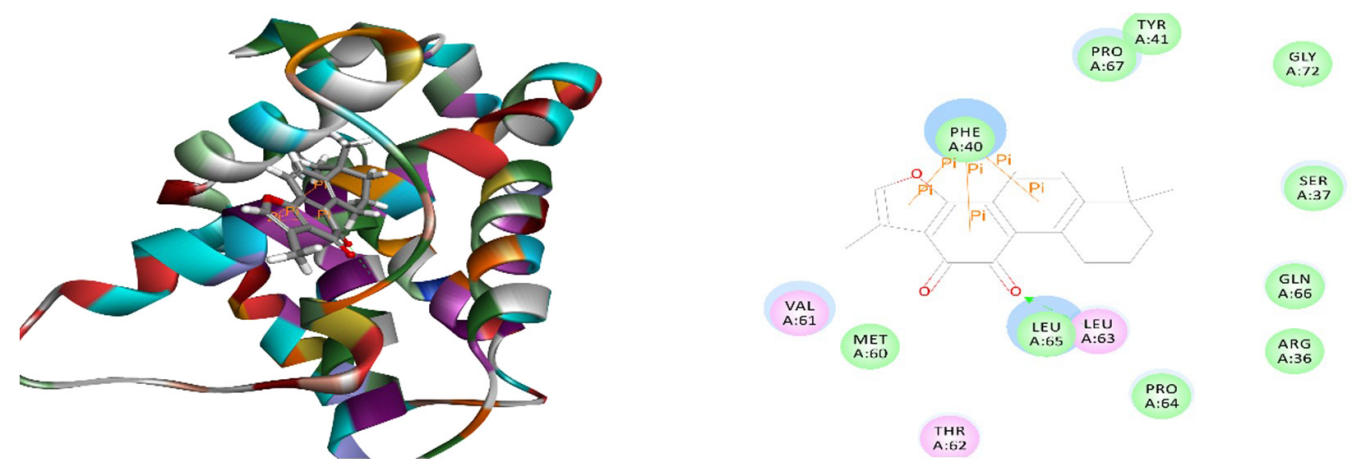

C
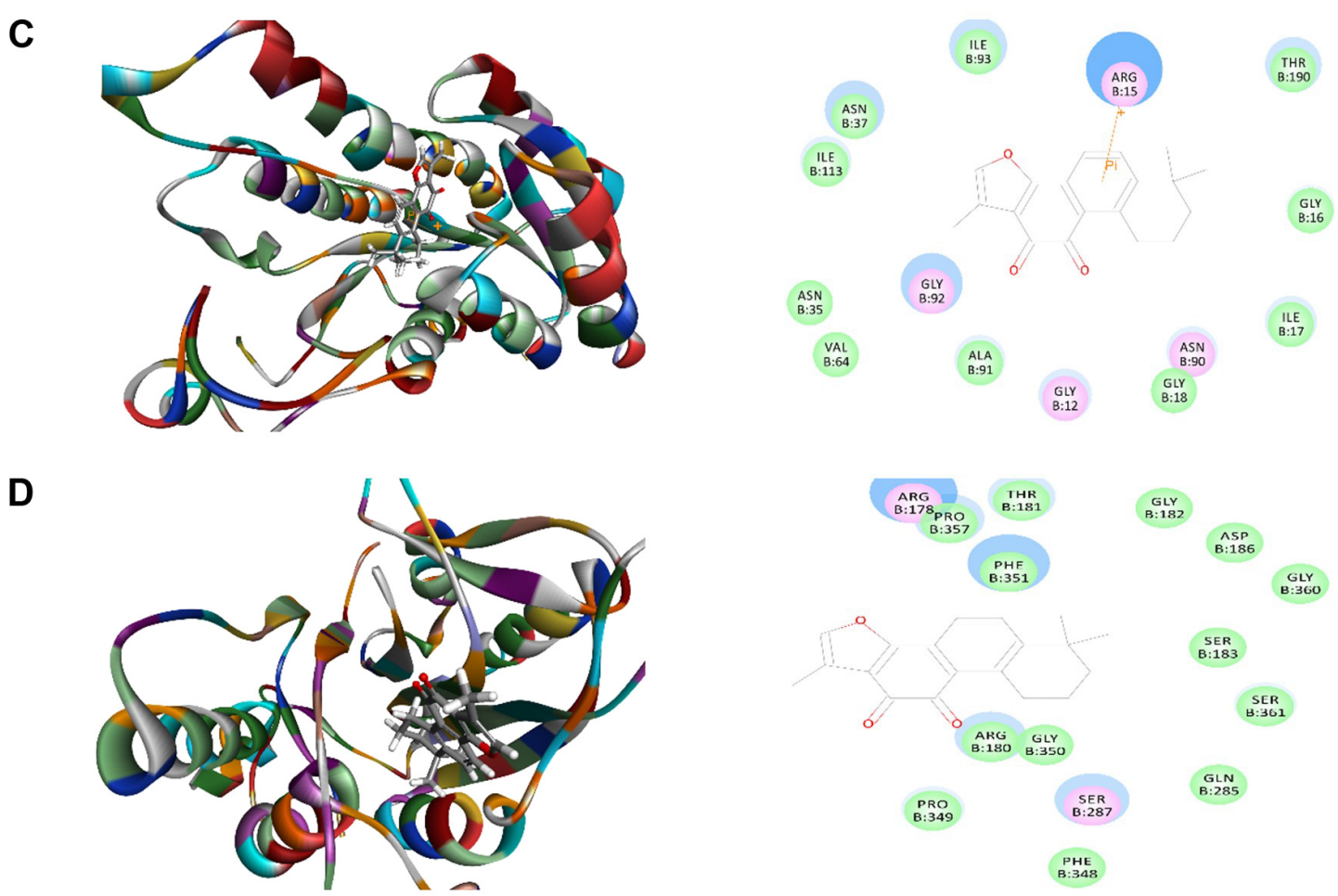

Figure 6 Molecular interactions between tanshinone IIA and human Bcl-xl, Bakl, procaspase 3, and caspase 9 determined using Discovery Studio ${ }^{\circledR}$ program 3.I. Notes: (A) Bcl-xl (PDB ID: 4TUH), (B) BakI (PDB ID: 2JCN), (C) procaspase 3 (PDB ID: 4JRO), and (D) caspase 9 (PDB ID: 2AR9).

Abbreviation: PDB ID, Protein Data Bank identifier.

Arg95. Moreover, salvianolic acid A bound to caspase 9 via hydrogen bond formation at Arg180, Thr181, and Gln285 and $\pi-\pi$ stacking at Arg178 and Arg180 (Figure 9). Similarly, salvianolic acid $\mathrm{C}$ was docked into the active sites of human Bcl-xl, procaspase 3, and caspase 9 (Figure 10A-C). The CDOCKER interaction energy ranged from 37.3485 to $52.9465 \mathrm{kcal} / \mathrm{mol}$ (Table 1). Salvianolic acid C bound to Bcl-xl via hydrogen bond formation at Arg102 and glu129 
A

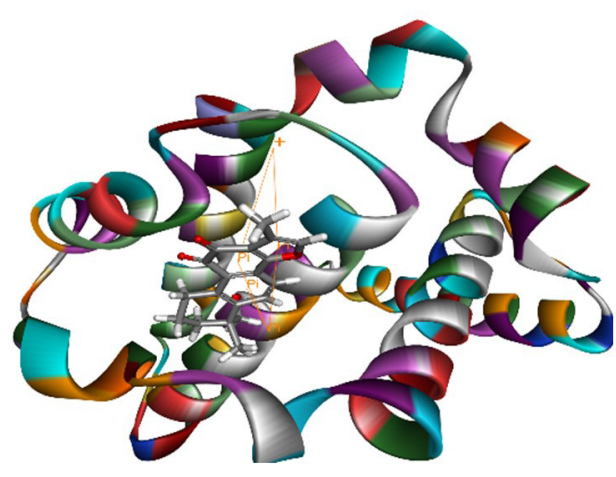

B

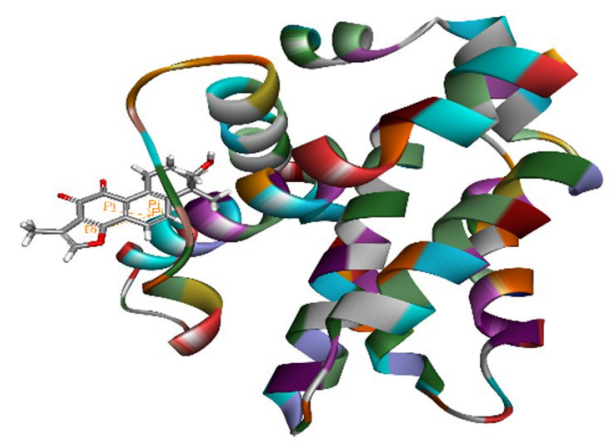

C

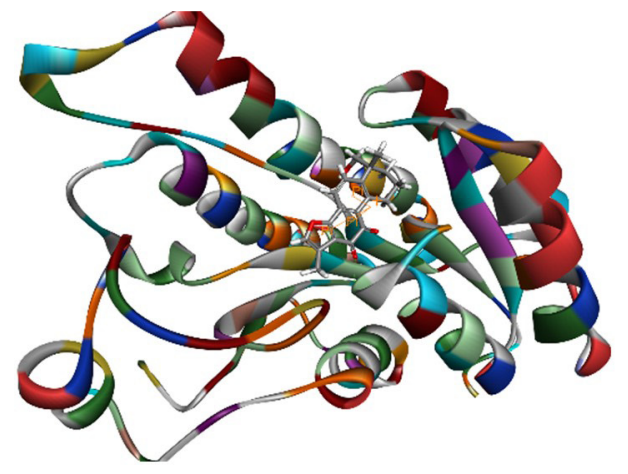

D

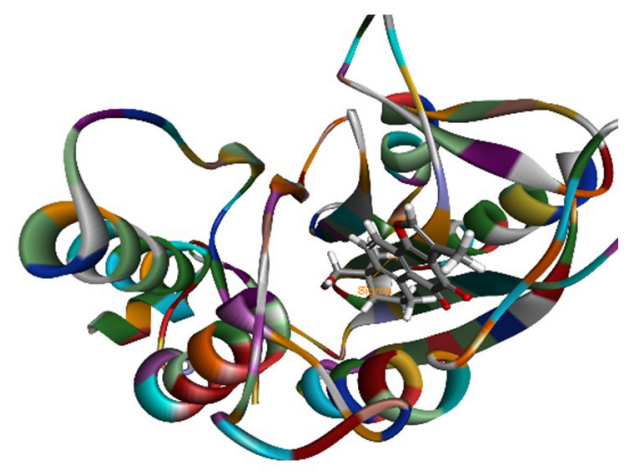

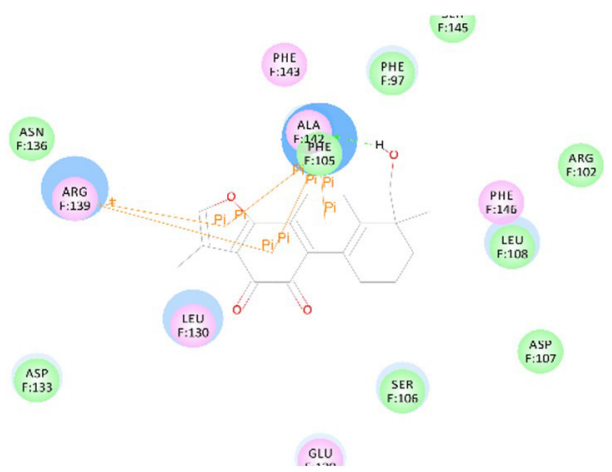
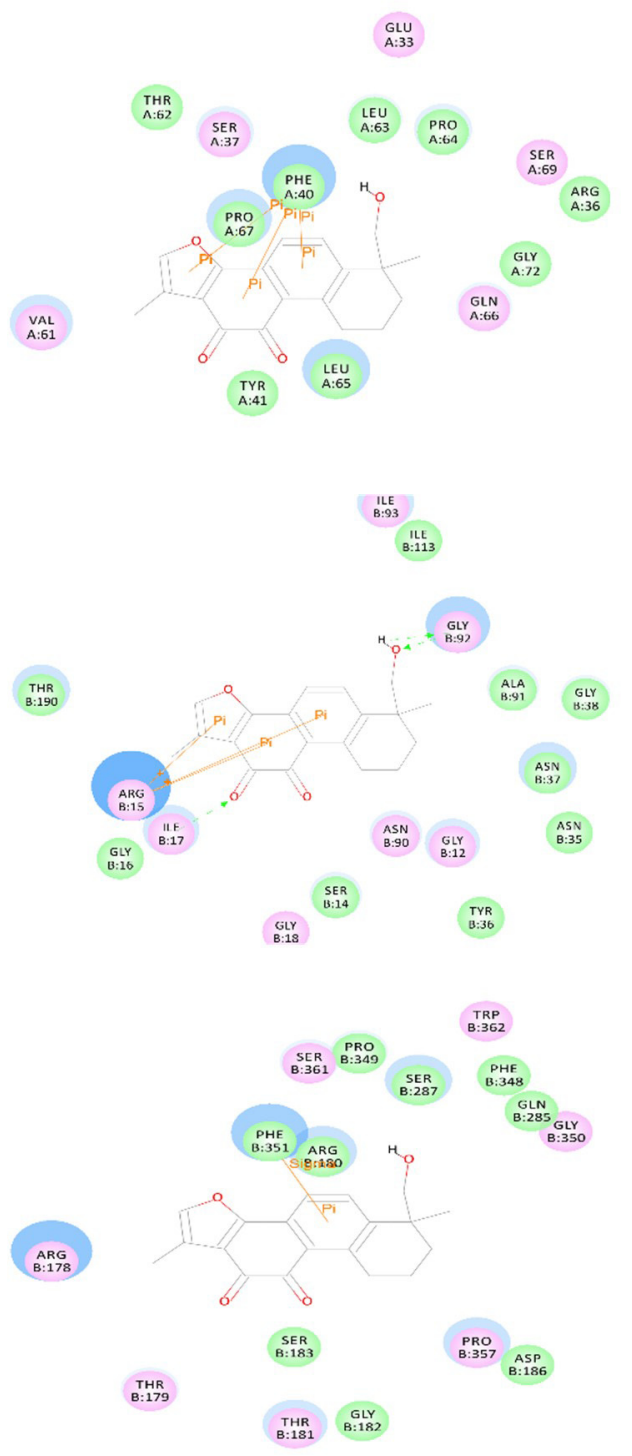

Figure 7 Molecular interactions between tanshinone IIB and human Bcl-xl, Bakl, procaspase 3, and caspase 9 determined using Discovery Studio ${ }^{\circledR}$ program 3.I. Notes: (A) Bcl-xl (PDB ID: 4TUH), (B) Bakl (PDB ID: 2JCN), (C) procaspase 3 (PDB ID: 4JRO), and (D) caspase 9 (PDB ID: 2AR9).

Abbreviation: PDB ID, Protein Data Bank identifier.

and $\pi-\pi$ stacking at $\operatorname{Arg} 102$. Salvianolic acid $C$ also formed seven hydrogen bonds with procaspase 3 at Gly18, Asn35, Asn37, Ala62, Val64, and Asn90, two charge interactions at $\operatorname{Arg} 15$ and Gly 38 , and two $\pi-\pi$ stacking at $\operatorname{Arg} 15$. Moreover, salvianolic acid $\mathrm{C}$ bound to caspase 9 via hydrogen bond formation at $\operatorname{Arg} 180$, Gln285, Ser287, and Gly360, charge interaction at Asp186, and $\pi-\pi$ stacking at $\operatorname{Arg} 178$ and Arg180 (Figure 10). 
A

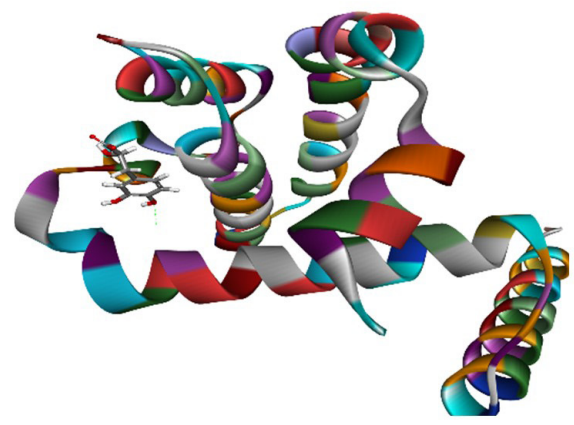

B

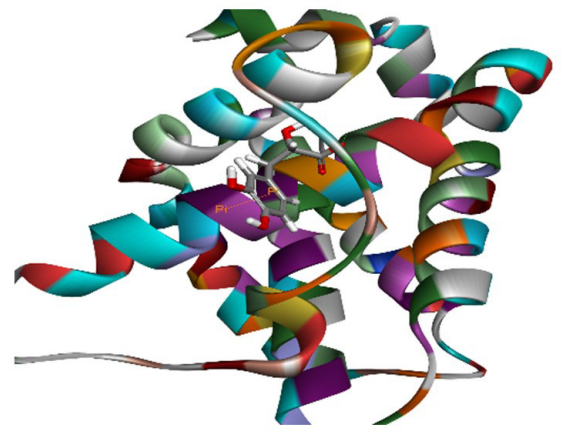

C
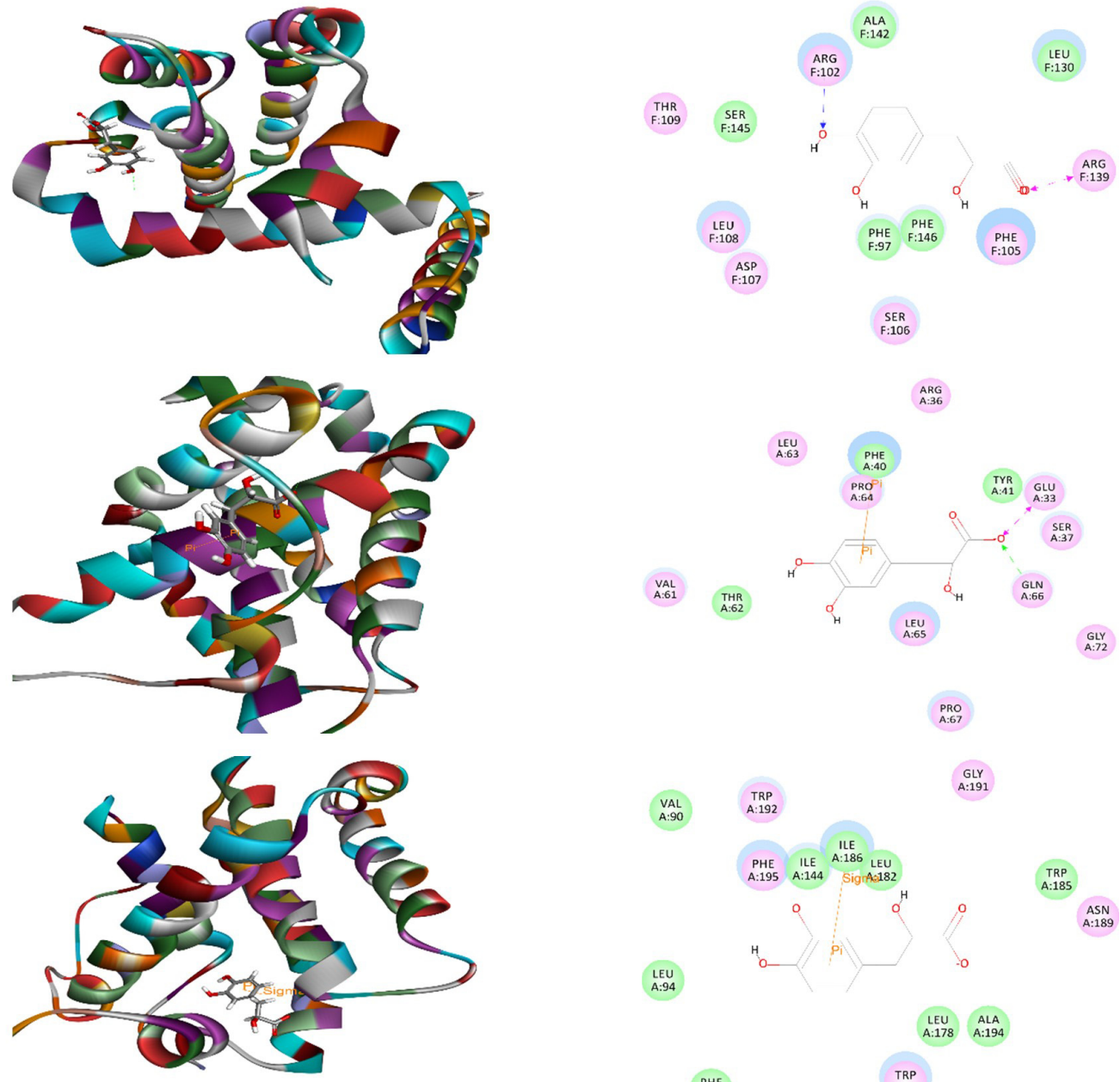

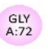

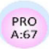

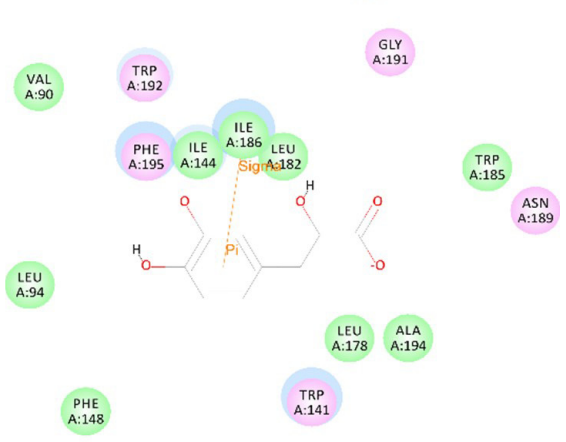

D

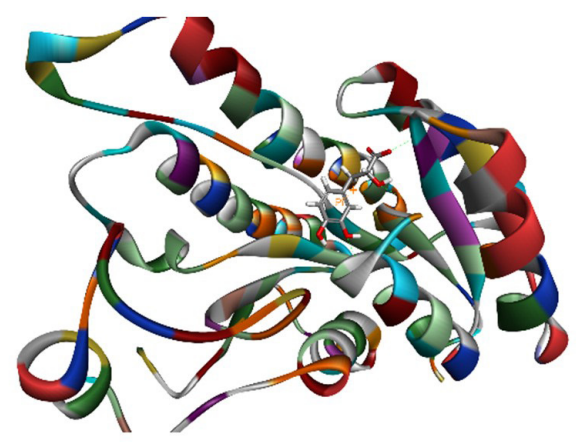

$\underset{\substack{116 \\ \text { s.6 }}}{9.93}$

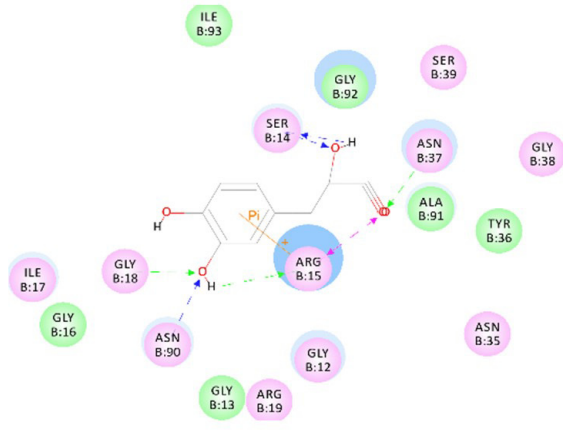

\section{$\mathbf{E}$}

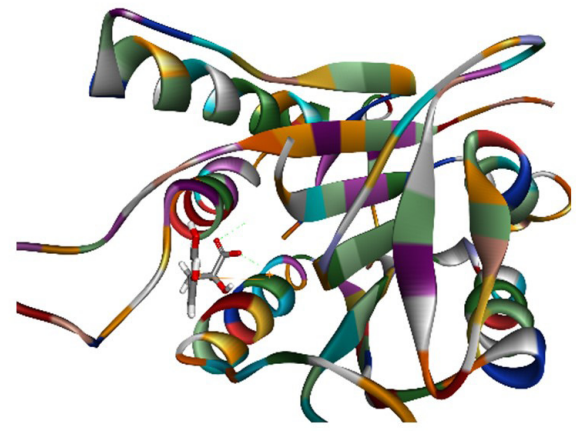

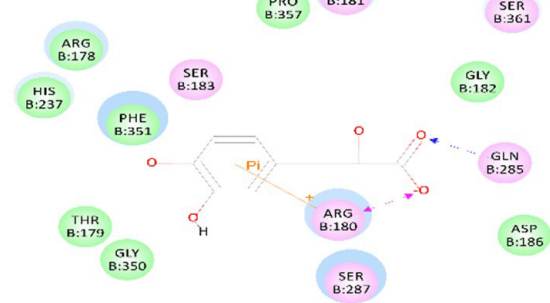

$\underset{\substack{P R O \\ 8: 349}}{P(2)}$

Figure 8 Molecular interactions between danshensu and human Bcl-xl, Bakl, Bcl-2, procaspase 3, and caspase 9 determined using Discovery Studio ${ }^{\circledR}$ program 3.I. Notes: (A) Bcl-xl (PDB ID: 4TUH), (B) BakI (PDB ID: 2JCN), (C) Bcl-2 (PDB ID: 2O2I), (D) procaspase 3 (PDB ID: 4JRO), and (E) caspase 9 (PDB ID: 2 AR9). Abbreviation: PDB ID, Protein Data Bank identifier. 
A

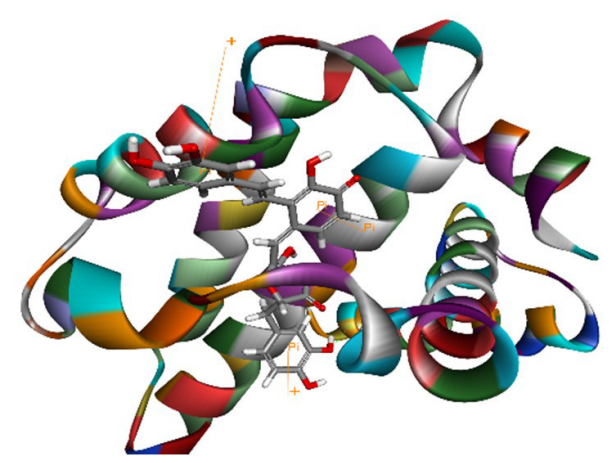

B

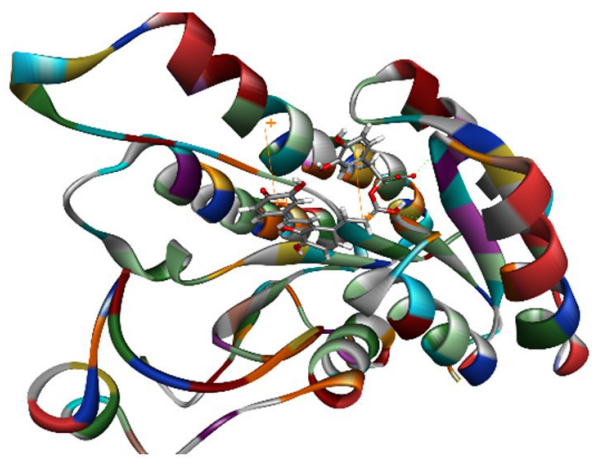

C

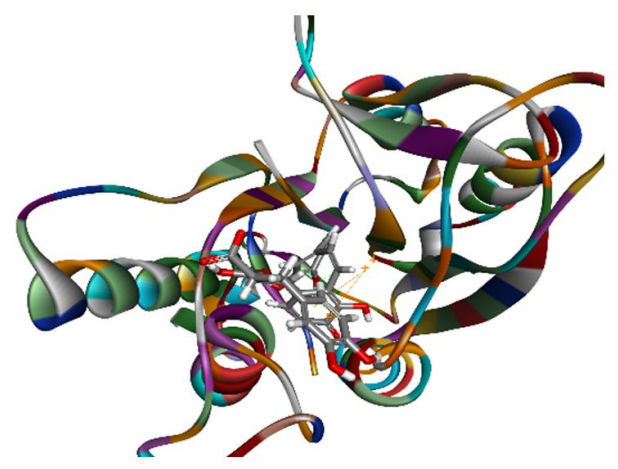

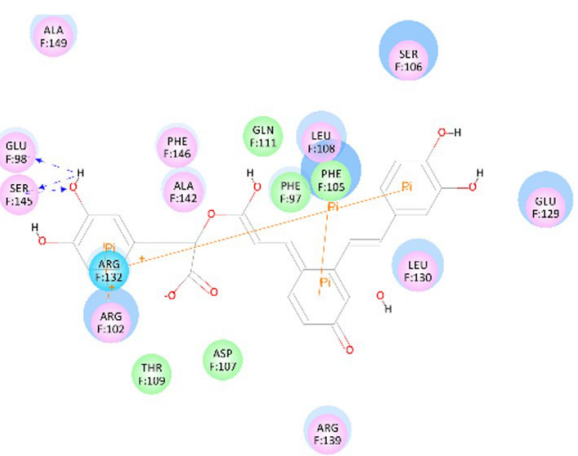
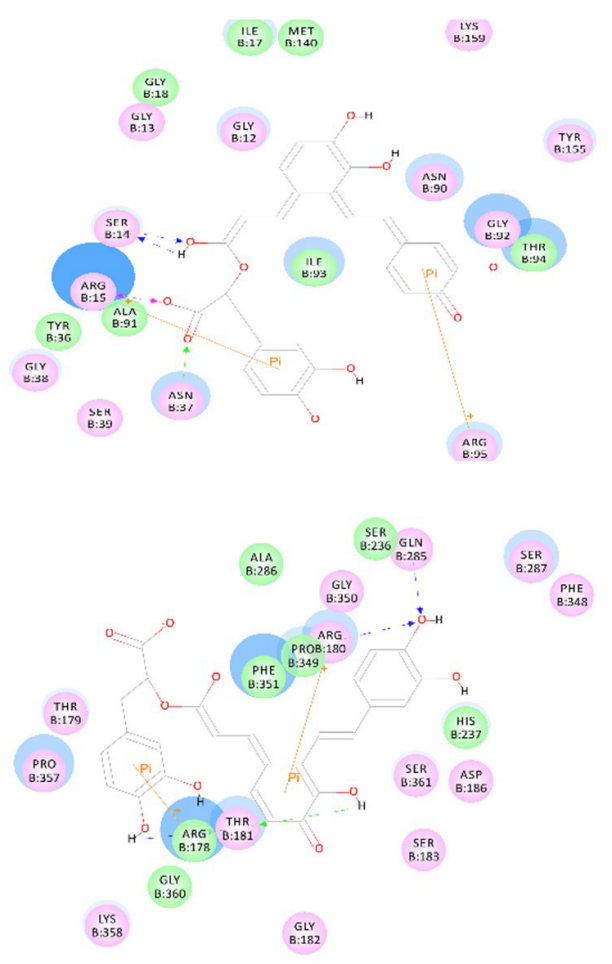

Figure 9 Molecular interactions between salvianolic acid A and human Bcl-xl, procaspase 3, and caspase 9 determined using Discovery Studio ${ }^{\circledR}$ program 3.I. Notes: (A) Bcl-xl (PDB ID: 4TUH), (B) procaspase 3 (PDB ID: 4JRO), and (C) caspase 9 (PDB ID: 2AR9).

Abbreviation: PDB ID, Protein Data Bank identifier.

Protocatechuic aldehyde was also docked into the active sites of human Bcl-xl, Bak1, procaspase 3, and caspase 9, with CDOCKER interaction energy ranging from 11.8606 to $24.6477 \mathrm{kcal} / \mathrm{mol}$ (Table 1 and Figure 11A-D). Protocatechuic aldehyde bound to $\mathrm{Bcl}-\mathrm{xl}$ via hydrogen bond formation at Arg102 and Ser145 and interacted with Bak1 via $\pi-\pi$ stacking at Phe 40 . Protocatechuic aldehyde also bound to procaspase 3 via hydrogen bond formation at Gly12, $\operatorname{Arg} 15$, Gly18, and Asn90 and via $\pi$ - $\pi$ stacking at Arg15. Moreover, there were two hydrogen bonds formed at Arg 180 and Asp 186 between protocatechuic aldehyde and caspase 9 . There was no hydrogen bond formation, charge interaction, or $\pi-\pi$ interaction between protocatechuic aldehyde and Bcl-2 (Figure 11). $\beta$-boswellic acid was also bound to procaspase
3 and caspase 9 via hydrogen bond formation at Arg15 and Arg178, respectively (Figure 12A and B). The CDOCKER interaction energy ranged from 31.6175 to $7.52648 \mathrm{kcal} /$ mol (Table 1).

Taken together, the results showed that ferulic acid, ligustilide, succinic acid, vanillic acid, tanshinone IIA, tanshinone IIB, danshensu, salvianolic acid A, salvianolic acid C, protocatechuic aldehyde, and $\beta$-boswellic acid could bind to active sites of human Bcl-xl, Bak1, Bcl-2, procaspase 3, and caspase 9 via hydrogen bond formation, charge interaction, and/or $\pi-\pi$ stacking. Ferulic acid, ligustilide, succinic acid, vanillic acid, tanshinone IIA, tanshinone IIB, danshensu, salvianolic acid $\mathrm{A}$, salvianolic acid $\mathrm{C}$, protocatechuic aldehyde, and $\beta$-boswellic acid may serve as enhancers or inhibitors of 
A

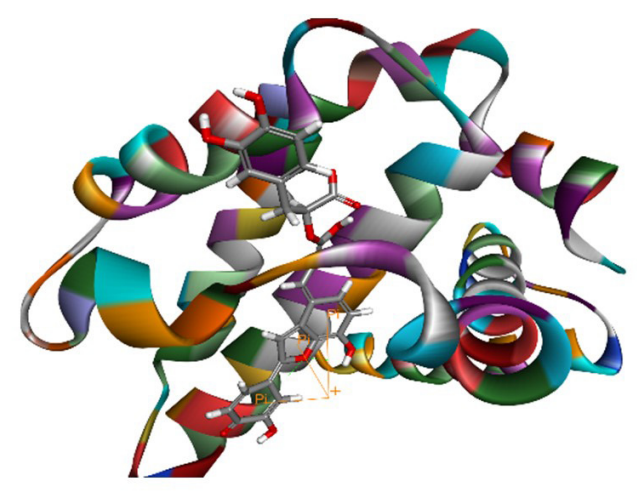

B

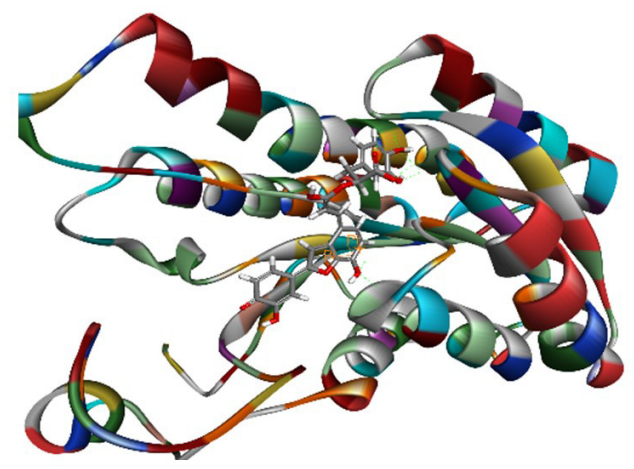

C

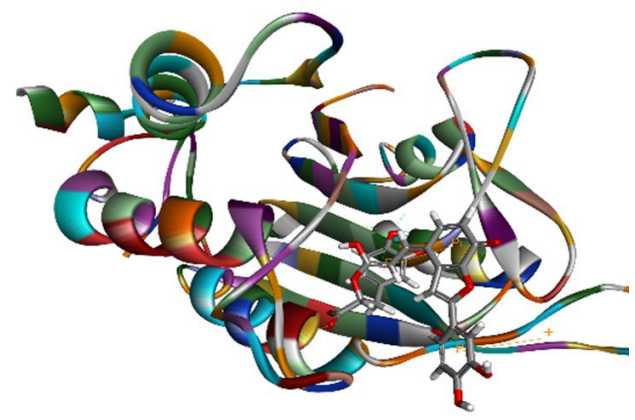

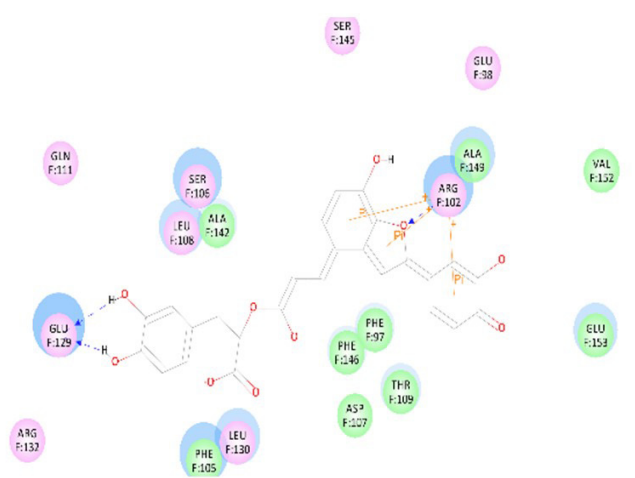
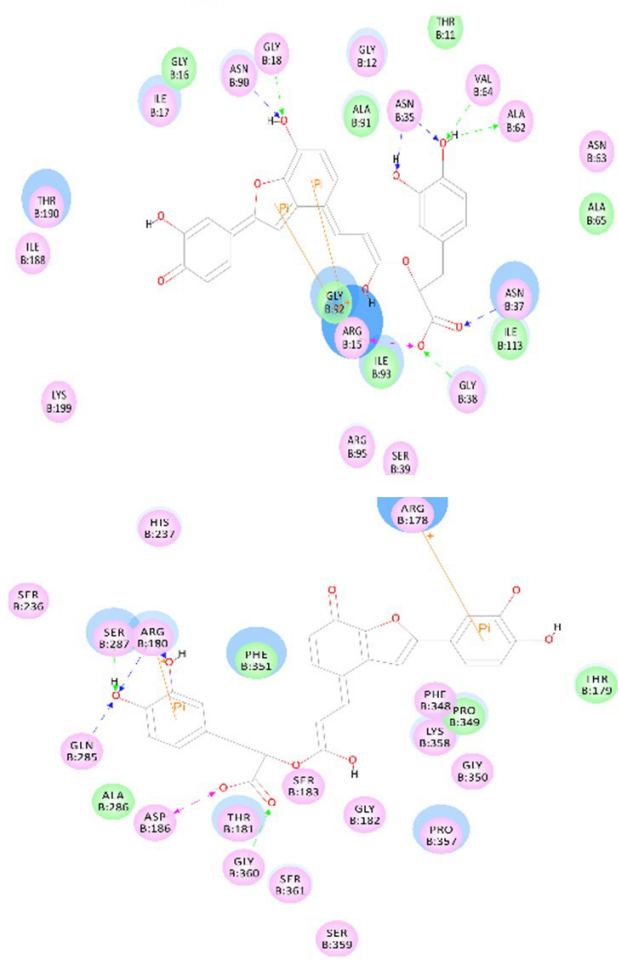

Figure 10 Molecular interactions between salvianolic acid $\mathrm{C}$ and human $\mathrm{Bcl}-\mathrm{xl}$, procaspase 3, and caspase 9 determined using Discovery Studio ${ }^{\circledR}$ program 3.I . Notes: (A) Bcl-xl (PDB ID: 4TUH), (B) procaspase 3 (PDB ID: 4JRO), and (C) caspase 9 (PDB ID: 2AR9).

Abbreviation: PDB ID, Protein Data Bank identifier.

these key regulators of apoptosis, contributing to the beneficial effects of HLXLD in the treatment of IHD.

\section{Predicted ADMET properties of the major active components of HLXLD}

The predicted ADMET properties of ferulic acid, ligustilide, succinic acid, vanillic acid, tanshinone IIA, tanshinone IIB, danshensu, salvianolic acid A, salvianolic acid B, salvianolic acid $\mathrm{C}$, protocatechuic aldehyde, and $\beta$-boswellic acid using Discovery Studio ${ }^{\circledR}$ program 3.1 are shown in Table 2. Ferulic acid, ligustilide, succinic acid, and vanillic acid had very good aqueous solubility. The aqueous solubility value [log (molar solubility)] of ferulic acid, ligustilide, succinic acid, and vanillic acid was between 4 and 5 (Table 2). However, they had a very low oral absorption. The blood-brain barrier permeability was between 3 and 4 . Moreover, all properties and optimal prediction space (OPS) components of ferulic acid, ligustilide, succinic acid, and vanillic acid were within expected ranges with regard to the CYP2D6 interaction, hepatotoxicity, and plasma protein binding (Table 2). Tanshinone IIA and tanshinone IIB showed ADMET profiles similar to those of ferulic acid, ligustilide, succinic acid, and vanillic acid, except for aqueous solubility and absorption. Tanshinone IIA and tanshinone IIA showed a very low aqueous solubility level of 0 , but a good absorption at 1-2.

There were various predictions for the ADMET profile of salvianolic acid A, salvianolic acid B, salvianolic acid $\mathrm{C}$, protocatechuic aldehyde, and $\beta$-boswellic acid, due to 
A

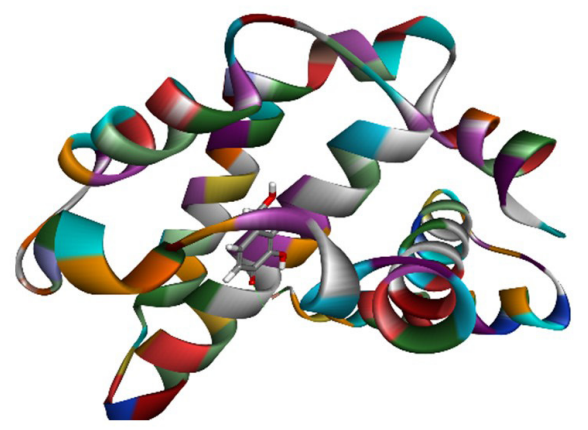

B

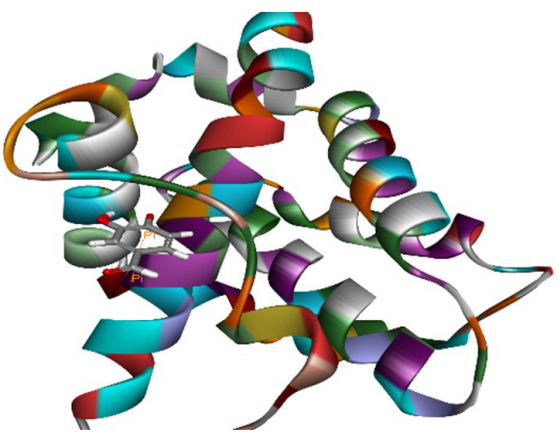

C

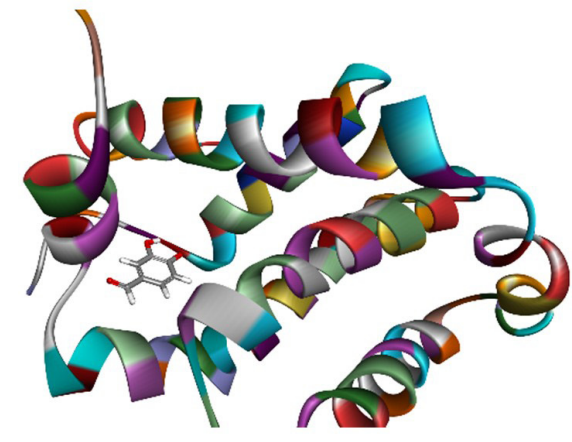

D

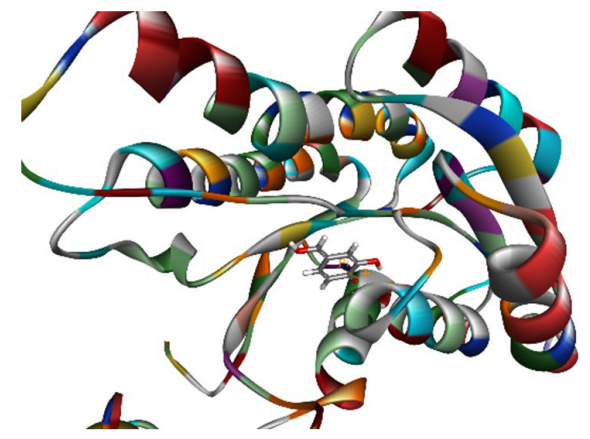

E

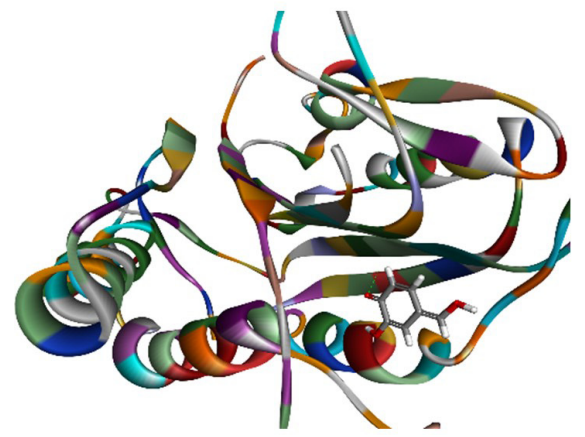

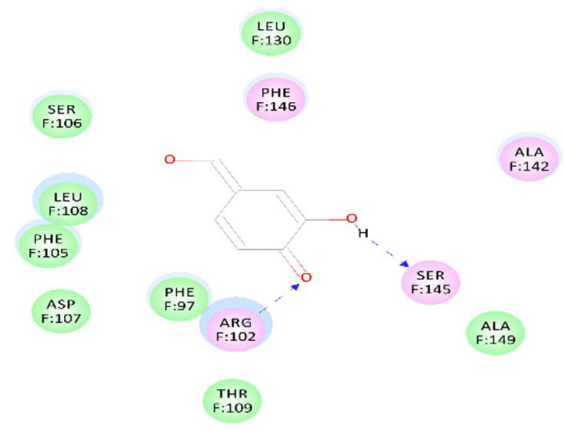

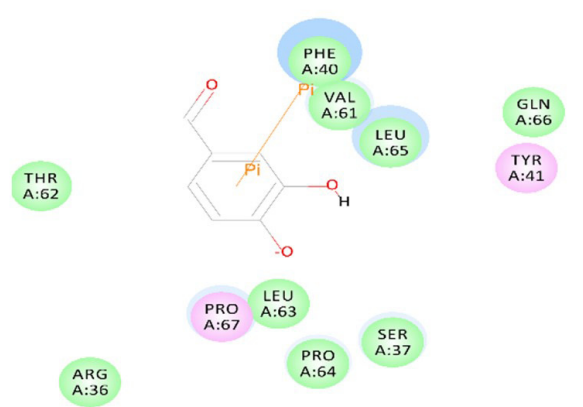

$\underset{\substack{\text { TRP } \\ \text { A:185 }}}{\substack{A L A \\ \text { A:194 }}}$

PHE

LEU

A:186
ALI
A:144
A:191

A:141

ז.THR

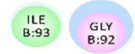

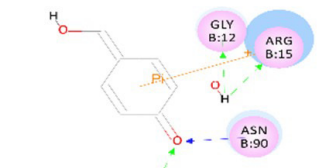

$\underset{\substack{\text { SER } \\ \text { S.in }}}{\ln }$

(IIE)

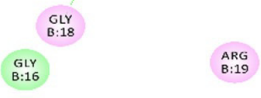

A:192

PHE

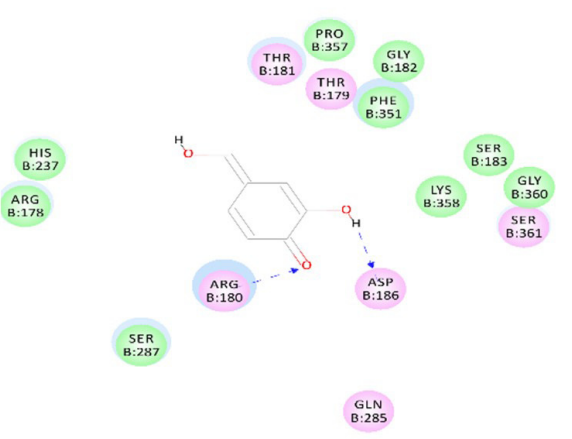

Figure I I Molecular interactions between protocatechuic aldehyde and human Bcl-xl, Bakl, Bcl-2, procaspase 3, and caspase 9 determined using Discovery Studio ${ }^{\circledR}$ program 3.I. Notes: (A) Bcl-xl (PDB ID: 4TUH), (B) BakI (PDB ID: 2JCN), (C) Bcl-2 (PDB ID: 2O2I), (D) procaspase 3 (PDB ID: 4JRO), and (E) caspase 9 (PDB ID: 2AR9). Abbreviation: PDB ID, Protein Data Bank identifier. 
A

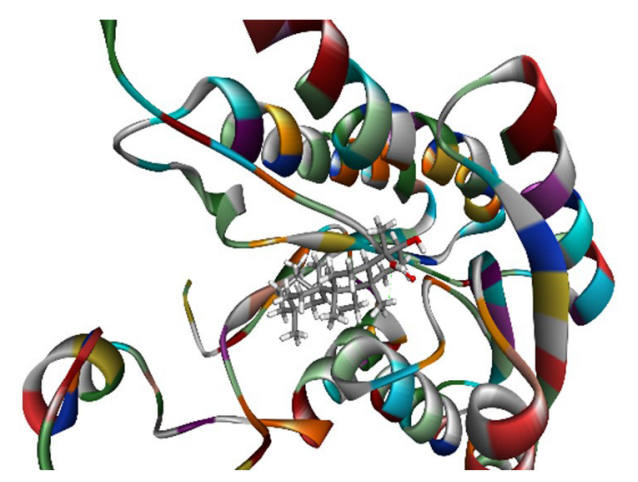

B

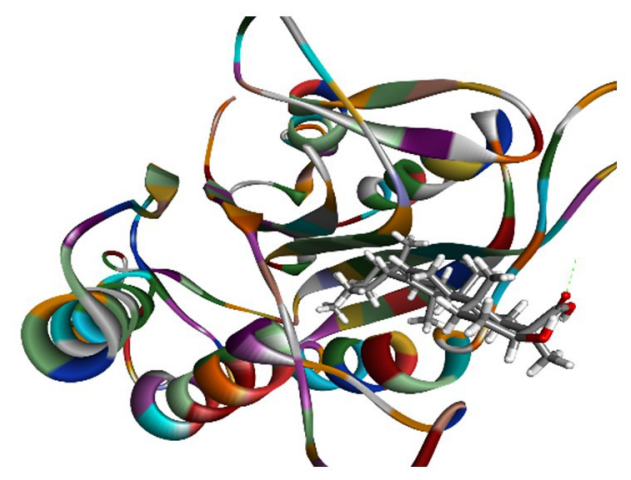

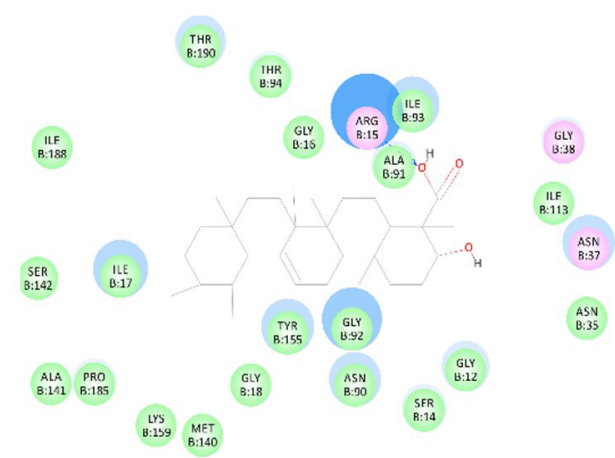

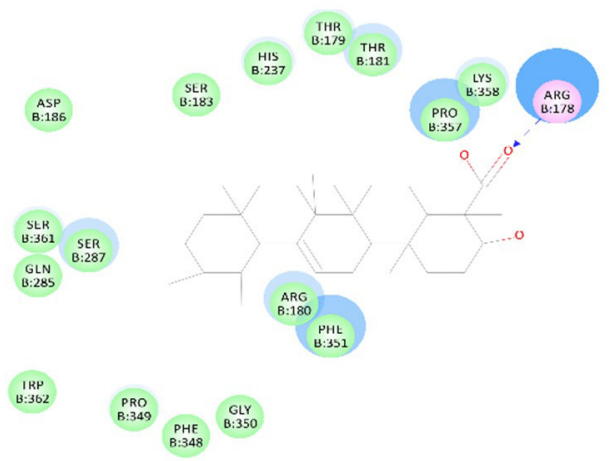

Figure 12 Molecular interactions between $\beta$-boswellic acid and human procaspase 3 and caspase 9 determined using Discovery Studio ${ }^{\circledR}$ program 3.1 . Notes: (A) Procaspase 3 (PDB ID: 4JRO) and (B) caspase 9 (PDB ID: 2AR9). Abbreviation: PDB ID, Protein Data Bank identifier.

the presence of multiple poses generated. There were 42 , 160, 52, 4, and 32 poses for salvianolic acid A, salvianolic acid $\mathrm{B}$, salvianolic acid $\mathrm{C}$, protocatechuic aldehyde, and $\beta$-boswellic acid, respectively (Table 2). Salvianolic acid A, salvianolic acid B, salvianolic acid C, and protocatechuic aldehyde showed high aqueous solubility and a low absorption. All properties and OPS components of salvianolic acid A, salvianolic acid B, salvianolic acid C, and protocatechuic aldehyde were within expected ranges with regard to the CYP2D6 interaction and plasma protein binding, except for hepatotoxicity (Table 2). $\beta$-boswellic acid showed a low aqueous solubility and a high absorption (Table 2).

\section{Effect of HLXLD on hemodynamic parameters in rats with coronary artery ligation-induced acute myocardial ischemia}

Next, we examined how HLXLD affected experimental IHD in rats. First, we examined the hemodynamic parameters in rats with coronary artery ligation-induced acute myocardial ischemia in the presence or absence of drug treatment. As shown in Tables 3 and 4, there was a marked decrease in MAP, LVSP, LVEDP, heart rate, and $\pm \mathrm{dp} / \mathrm{dt}_{\text {max }}$ in the model group of rats with coronary artery ligation-induced acute myocardial ischemia $(P<0.05$ by one-way ANOVA), compared with the sham group. Administration of HLXLD marked improved the hemodynamic profile with a significant increase in MAP, LVSP, heart rate, and $\pm \mathrm{dp} / \mathrm{dt}_{\max }$, compared with the model group. Moreover, the positive control group of rats administered isosorbide mononitrate $0.2 \mathrm{mg} / \mathrm{kg}$ also showed a marked beneficial effect on hemodynamics when compared with the model group. Taken together, HLXLD showed marked beneficial effects on coronary artery ligationinduced acute myocardial ischemia in rats.

\section{Effect of HLXLD on serum enzymes in rats with coronary artery ligation- induced acute myocardial ischemia}

Blood samples were analyzed after the rats has been treated for 4 weeks and the effect of HLXLD on key serum enzymes (SOD, CK, and LDH) was tested. There are three types of SOD in humans, including SOD1 in the cytoplasm, ${ }^{24}$ SOD2 in the mitochondria, ${ }^{25}$ and extracellular SOD3. ${ }^{26}$ SODs have an important role in the maintenance of redox homeostasis and have been implicated in the pathogenesis of several diseases, including cardiovascular disease. ${ }^{27}$ $\mathrm{CK}$ is a biomarker of myocardial infarction, and there are three isoenzymes of $\mathrm{CK}$, including CK-MM, CK-BB, and CK-MB. ${ }^{28,29} \mathrm{LDH}$ is of medical significance, acting as a 


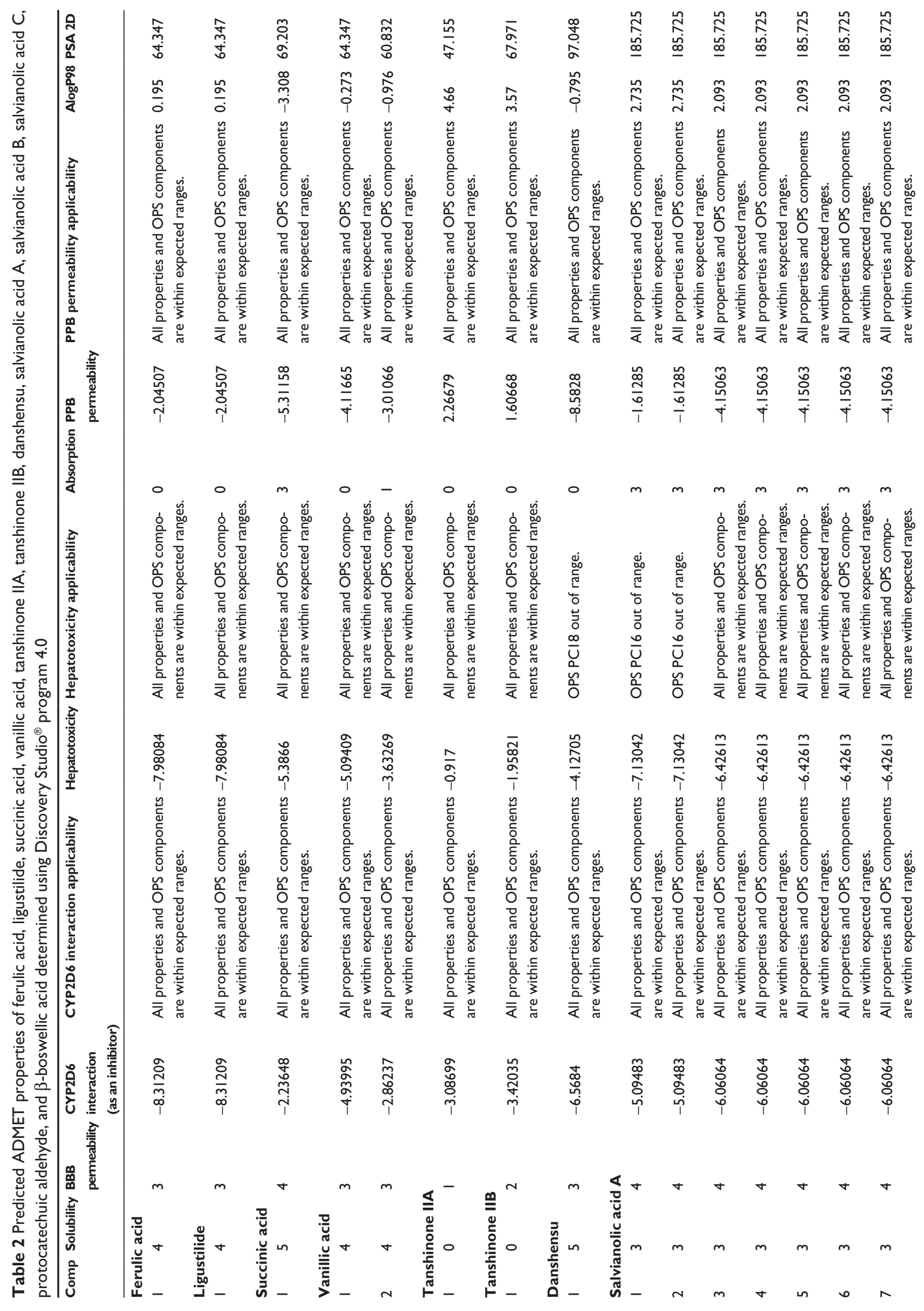


究

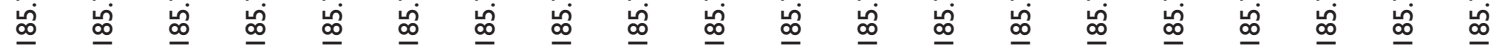

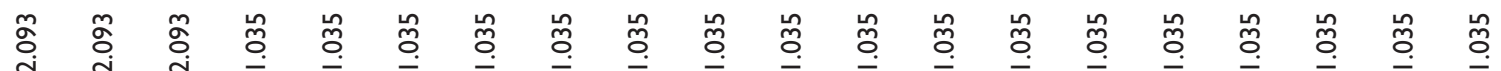

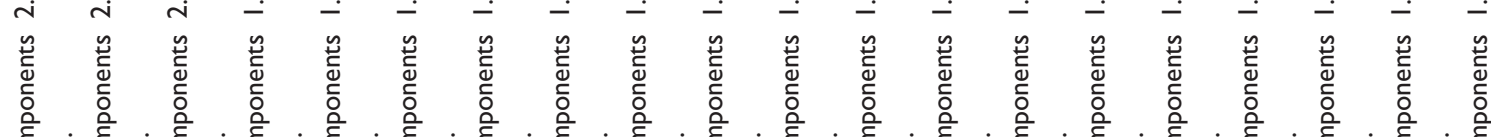

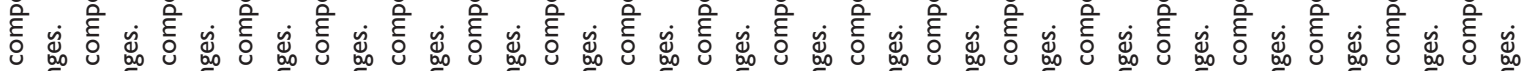

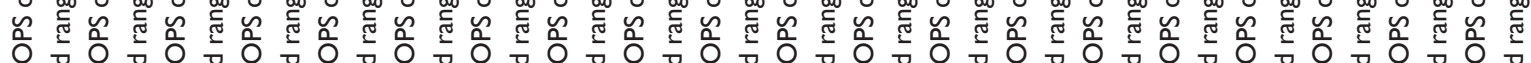

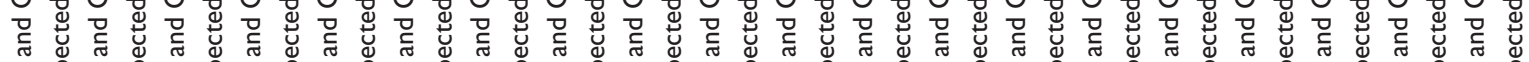

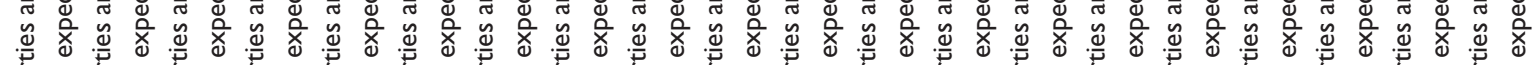

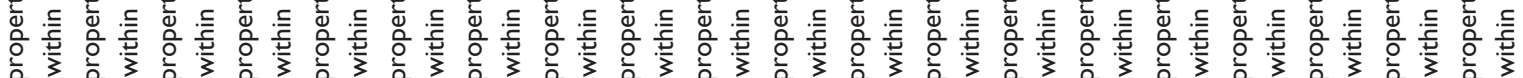

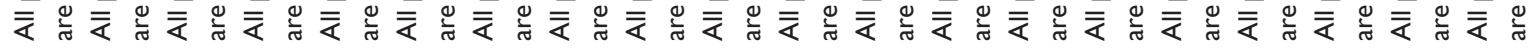

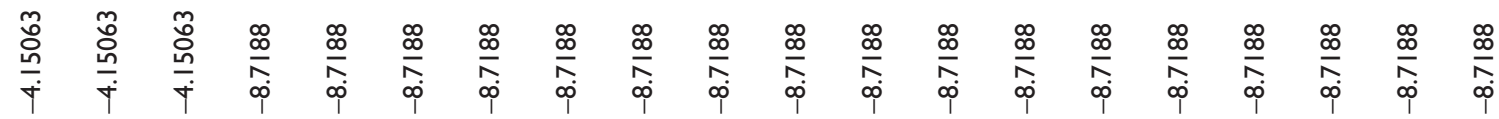

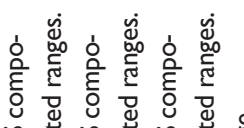

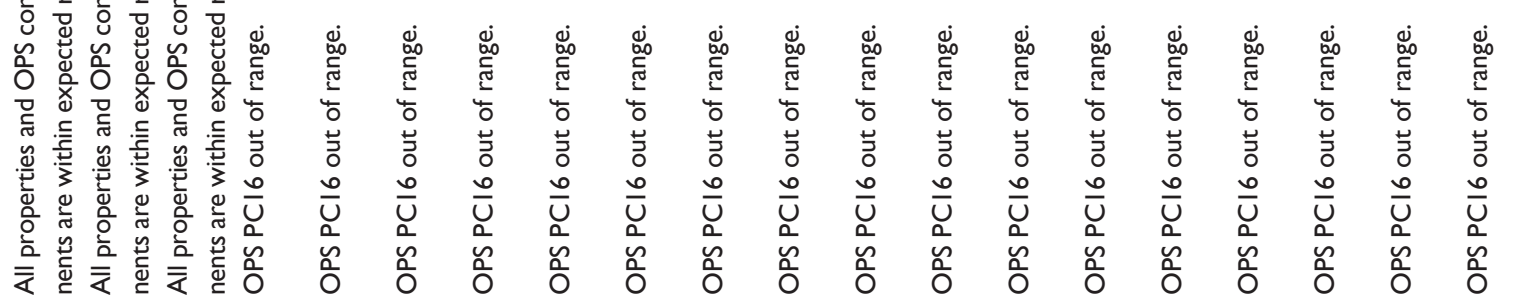

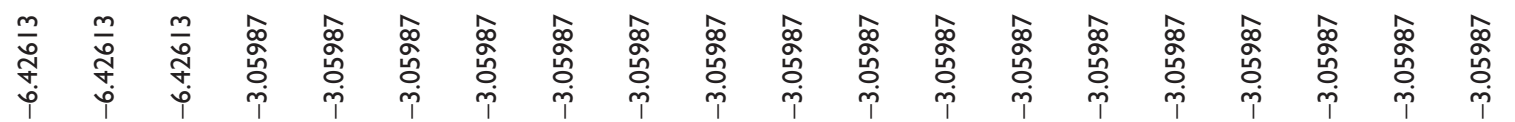

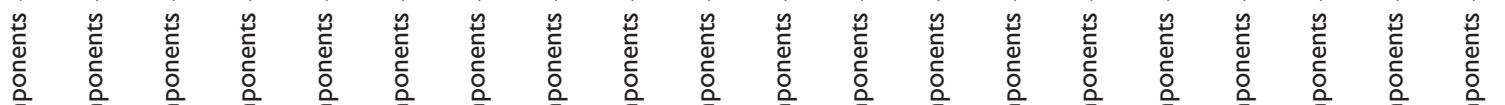

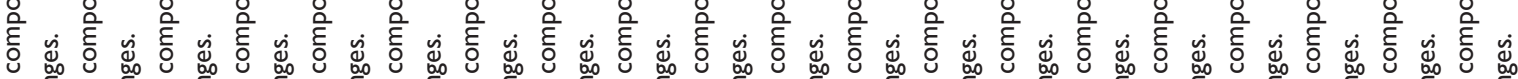

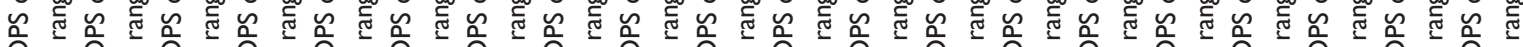

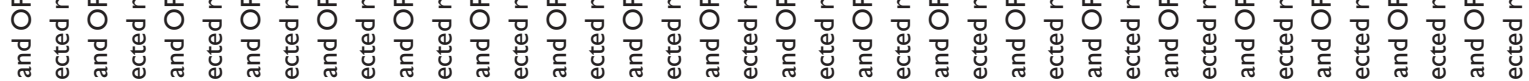

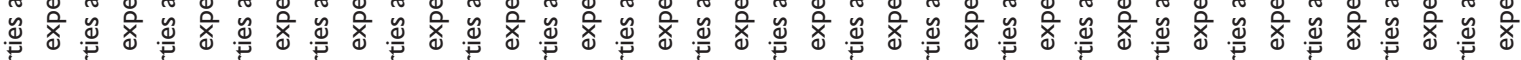

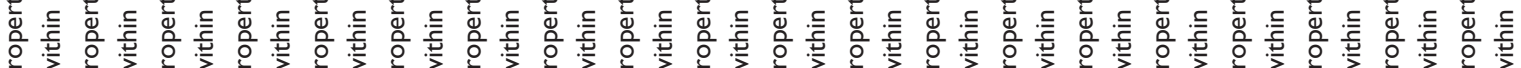

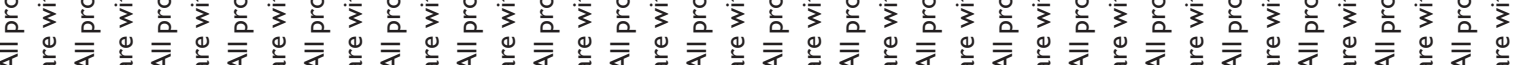

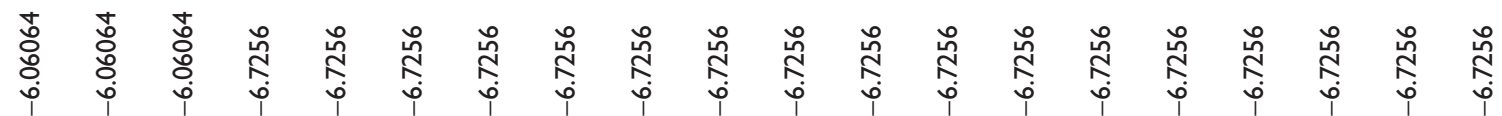

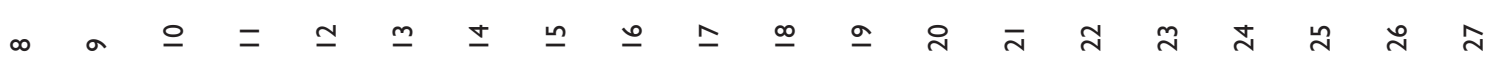




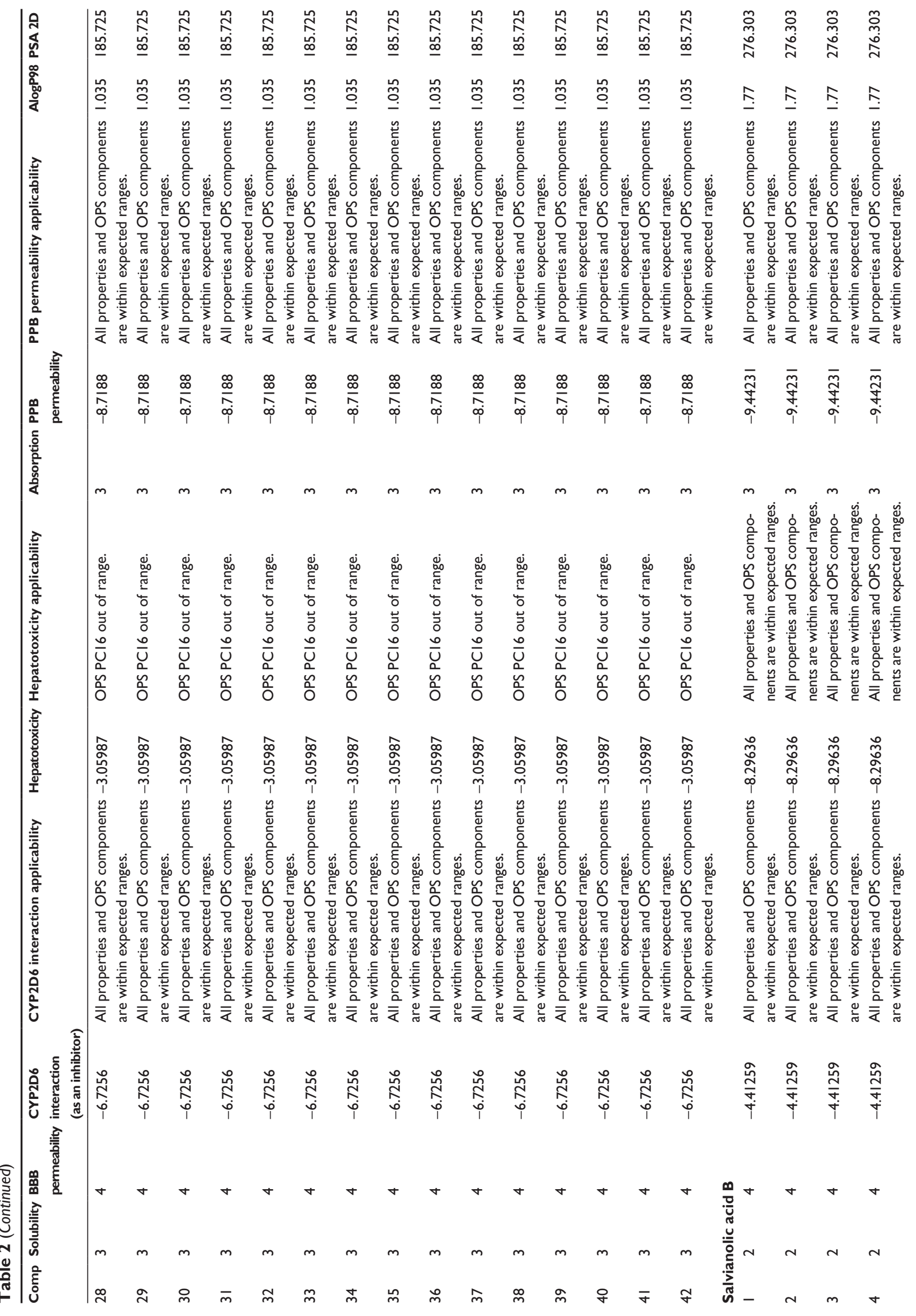




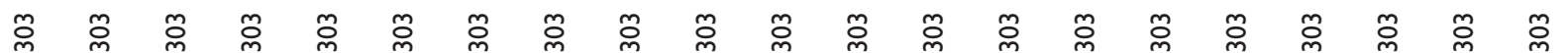

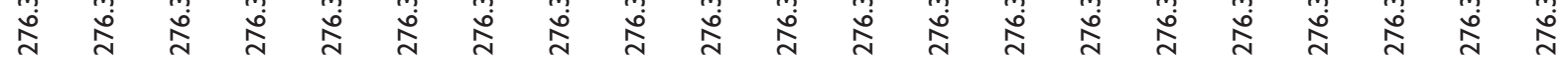

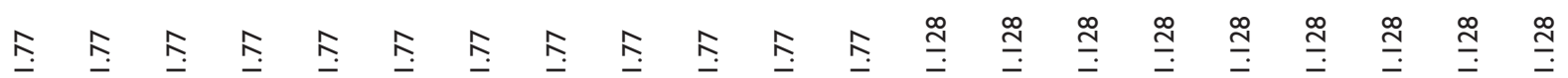

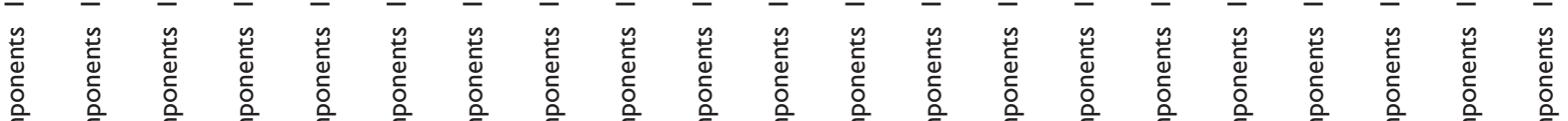

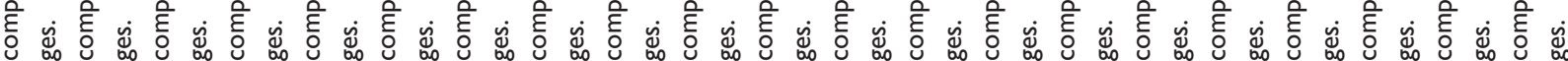

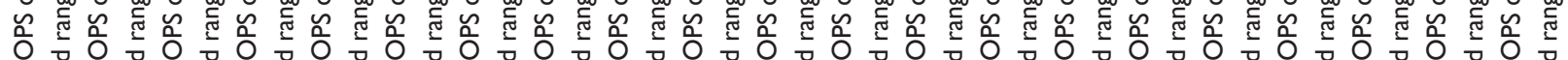

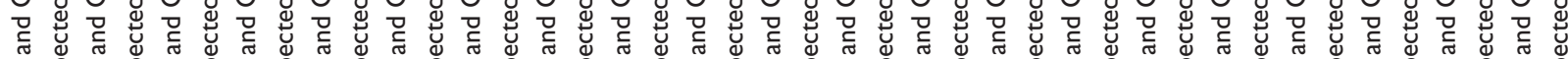

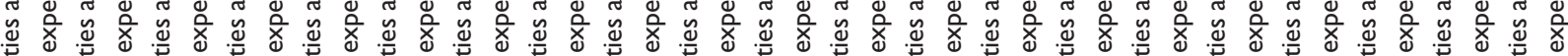

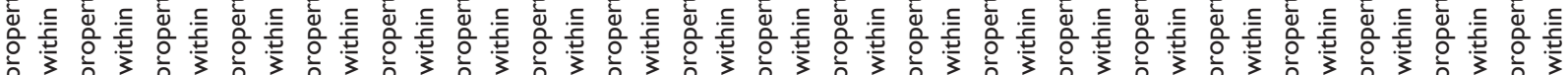

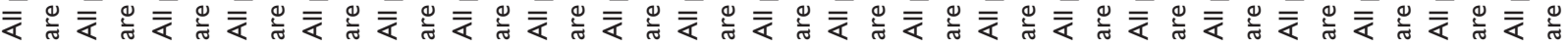

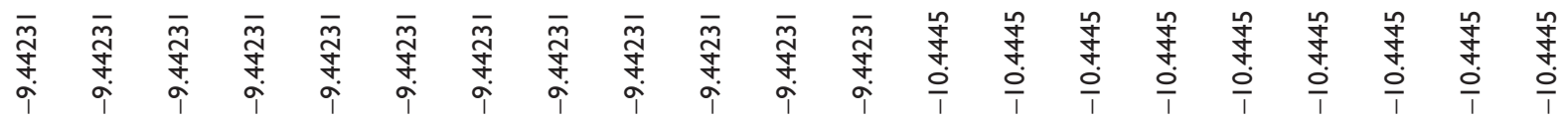

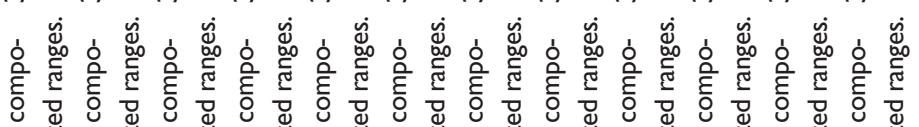

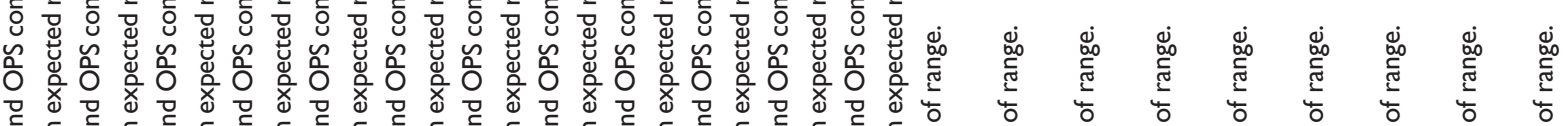

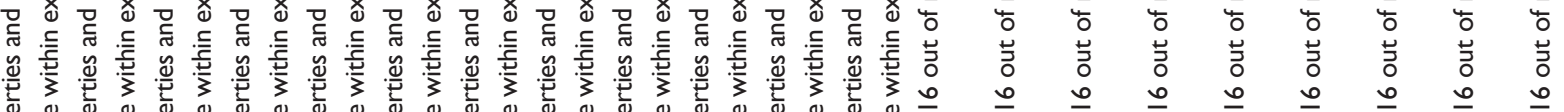

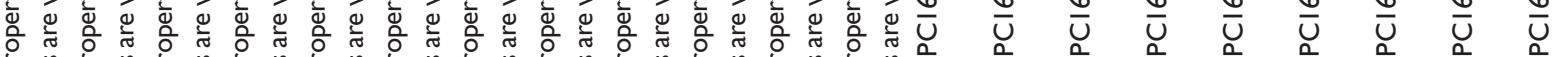

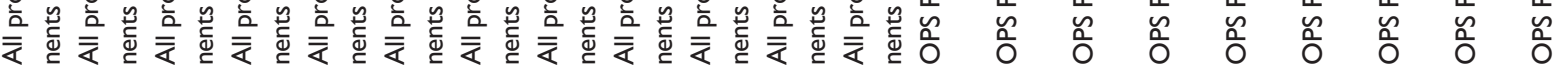

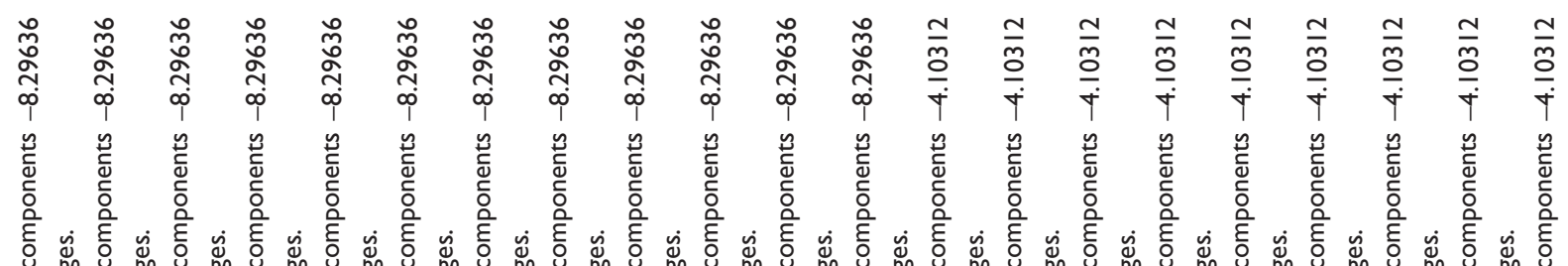
U.

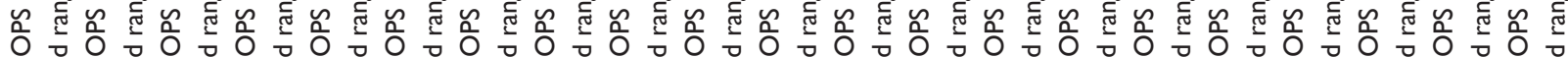

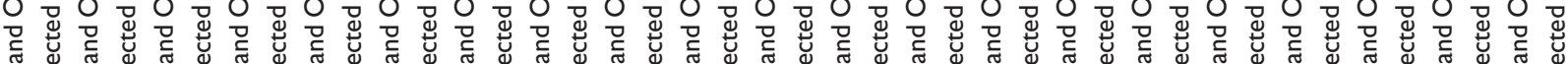

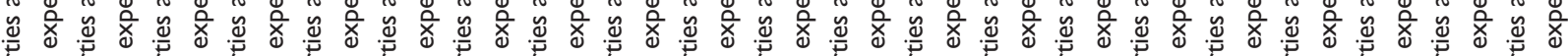
至 至

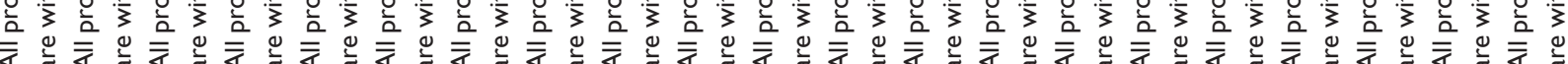

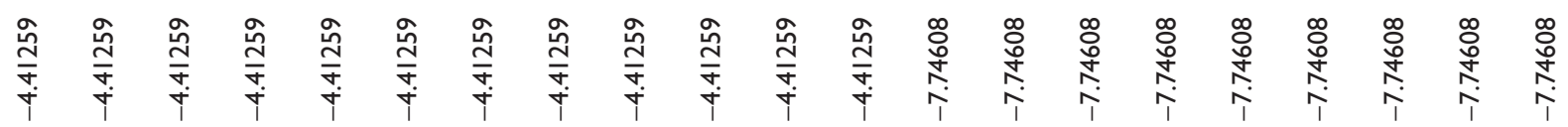

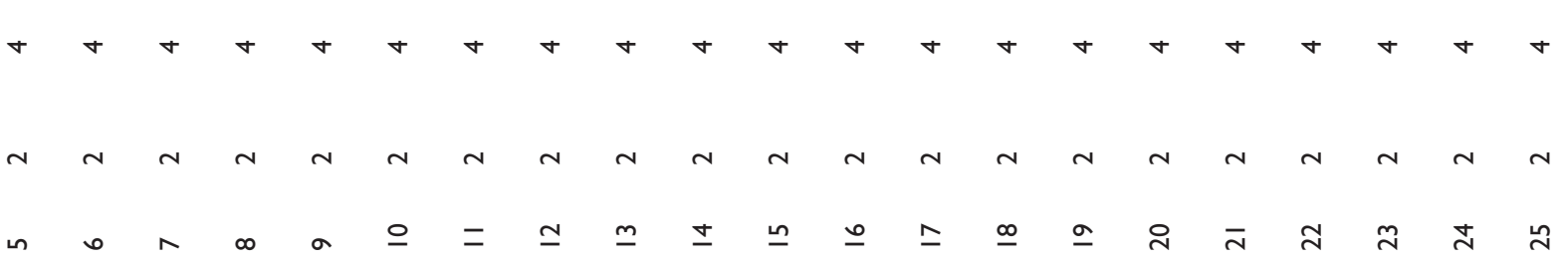




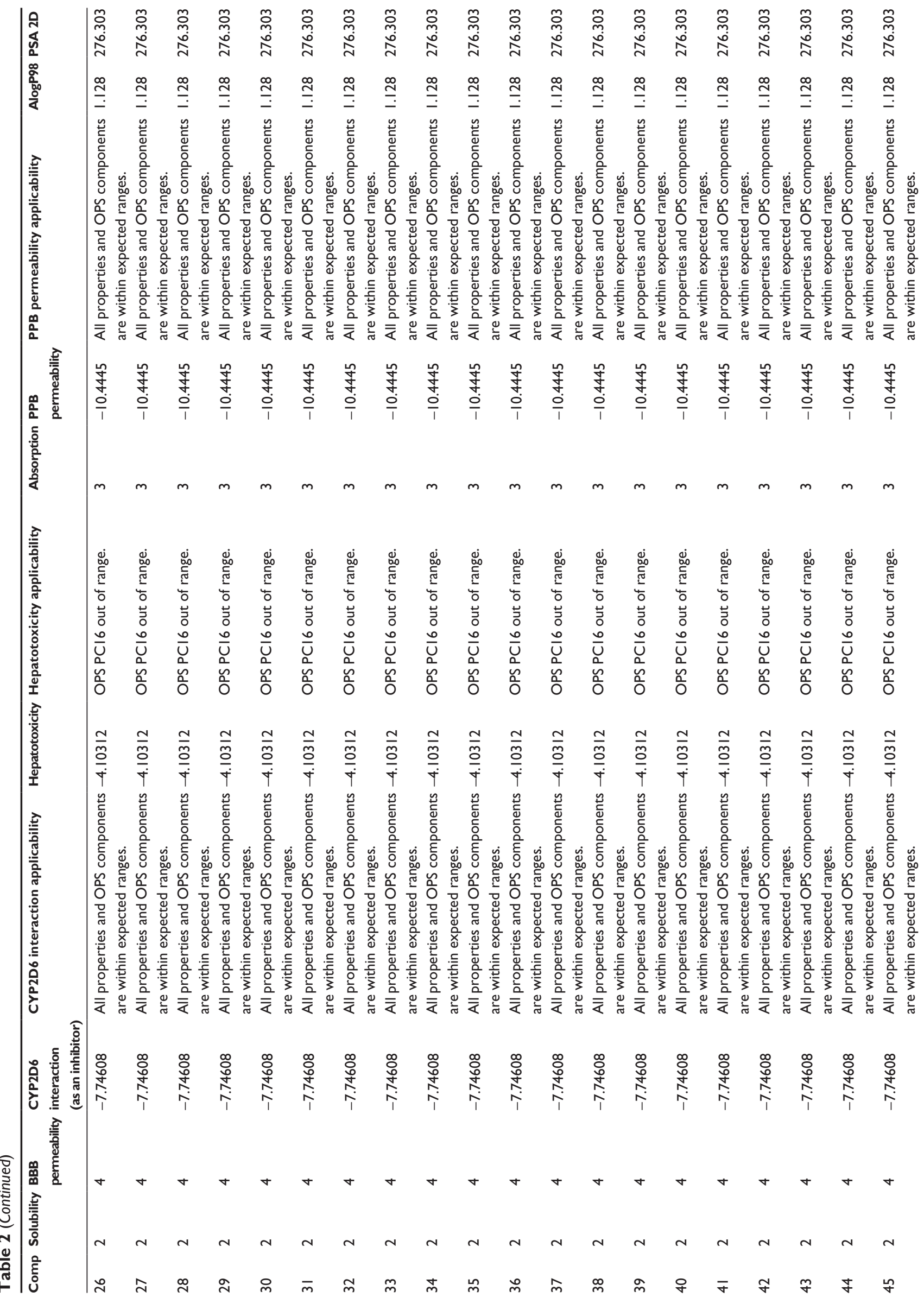




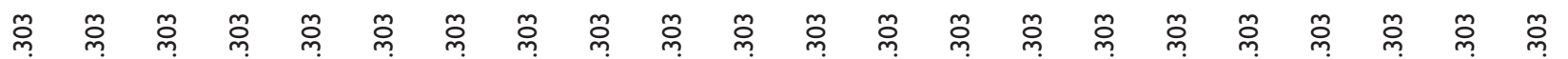
ป స

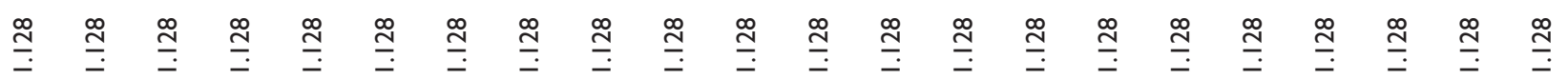

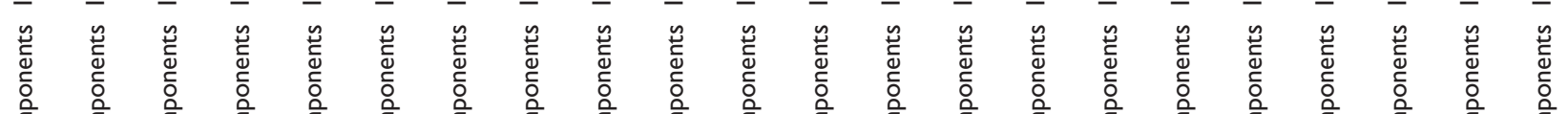

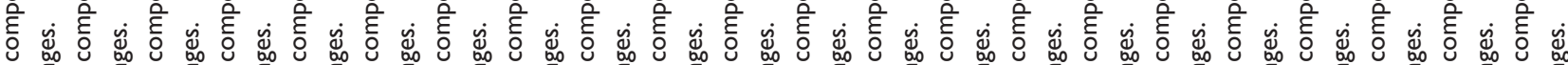

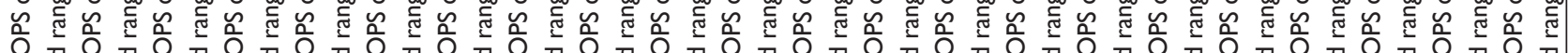

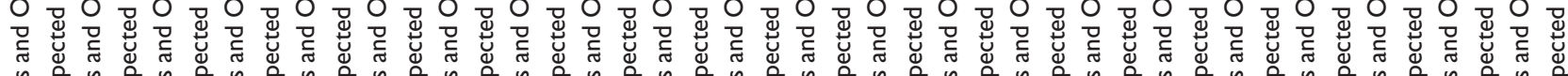

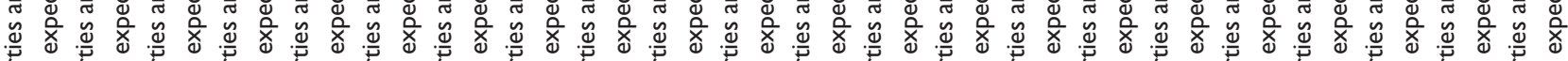

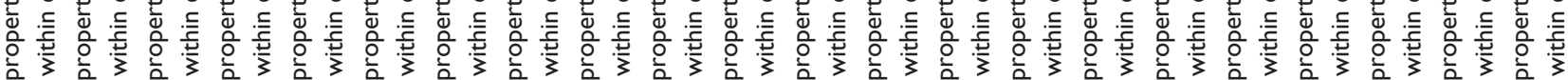

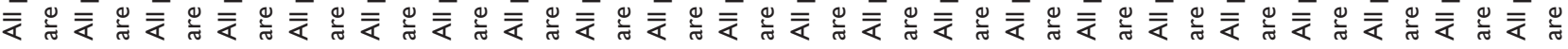

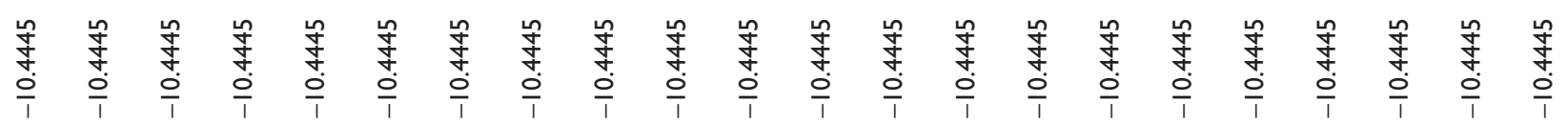

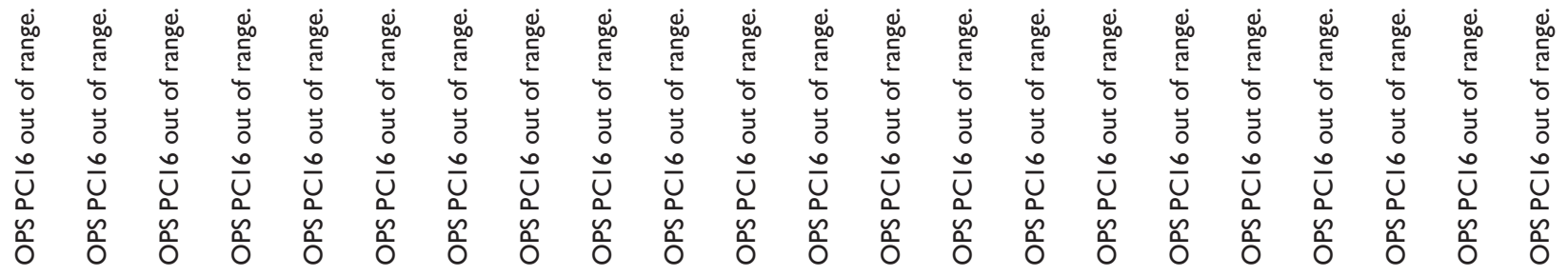

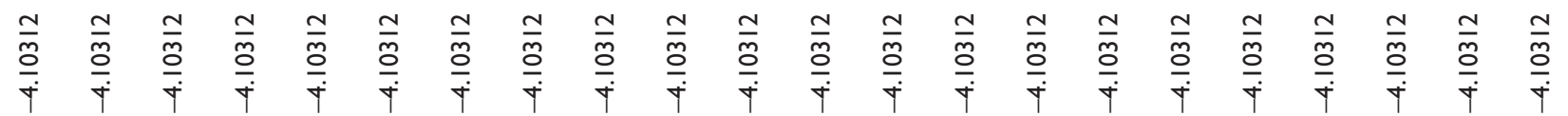

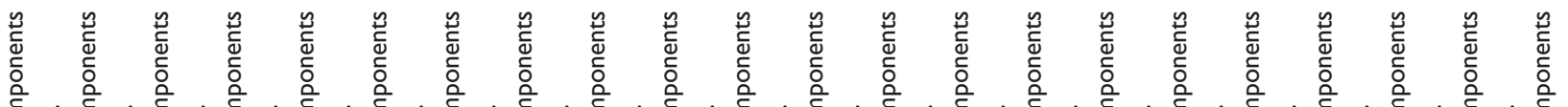

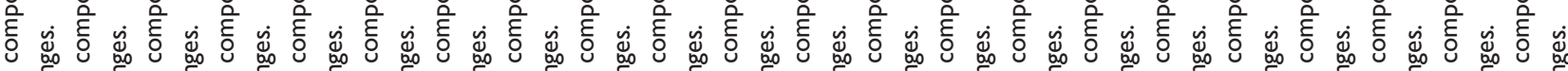

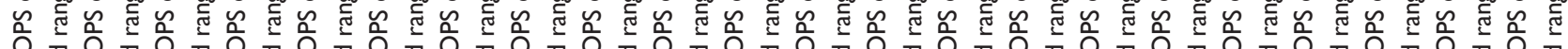

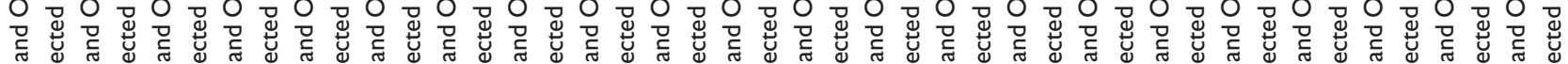

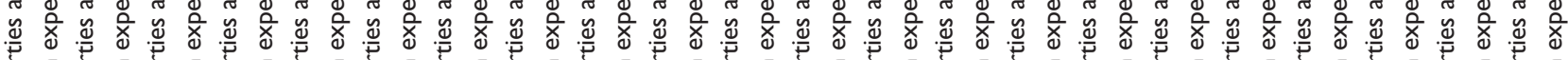

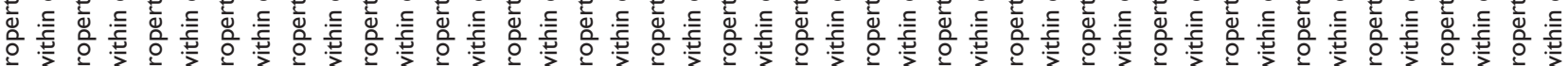

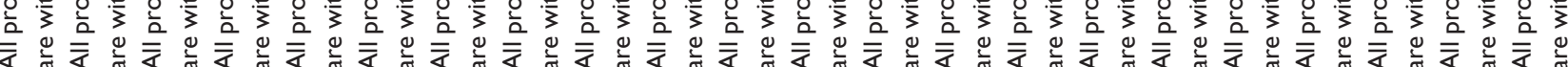

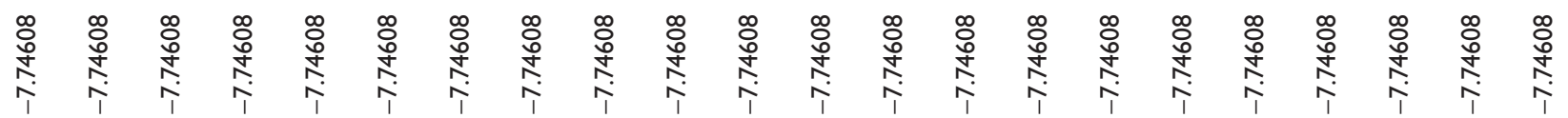

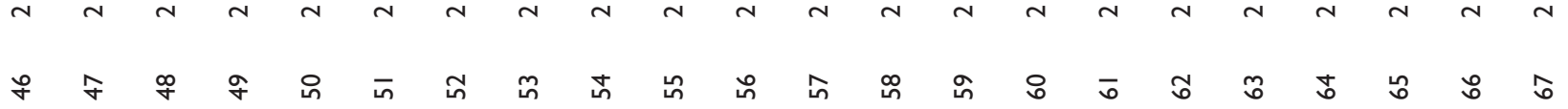




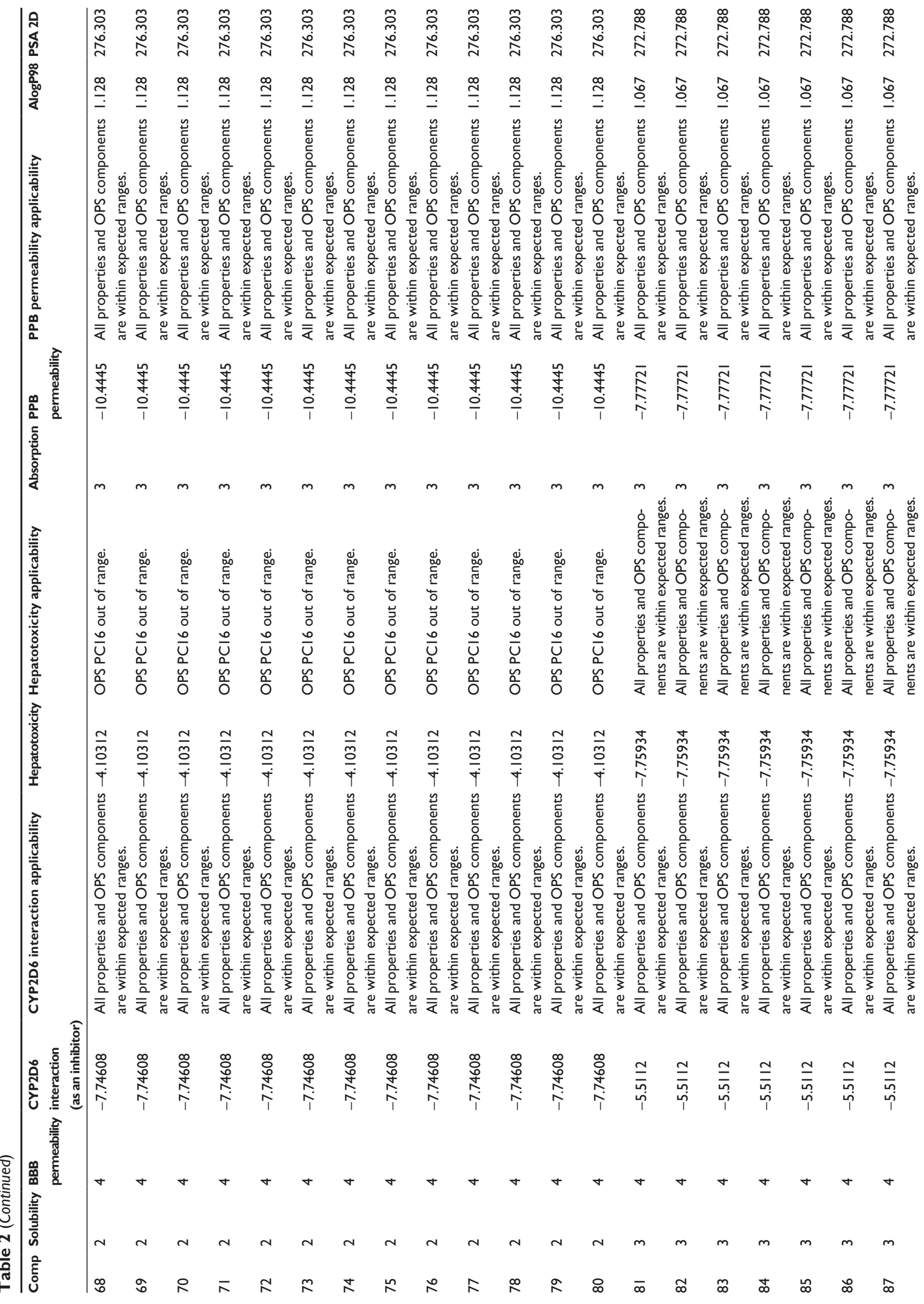




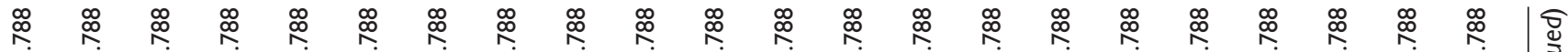

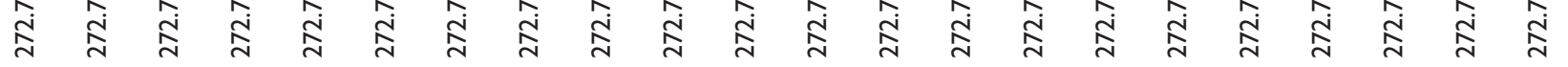
๖ి

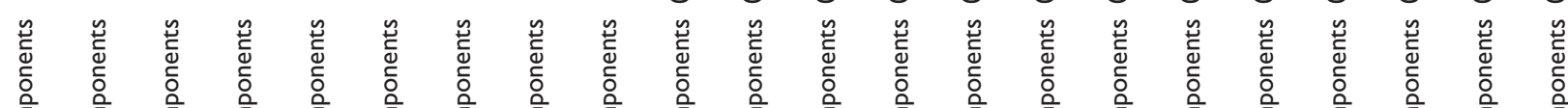

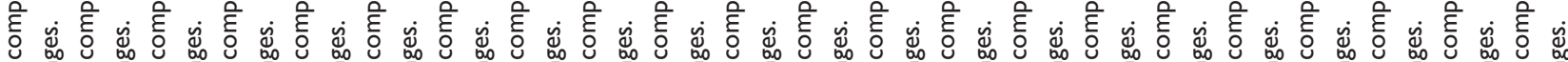

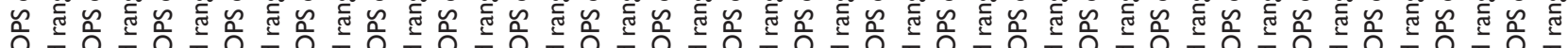

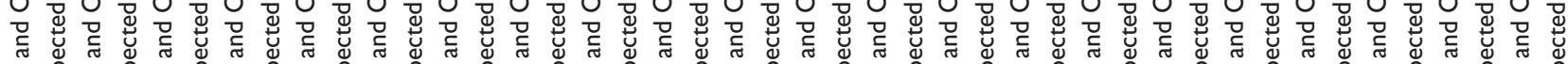

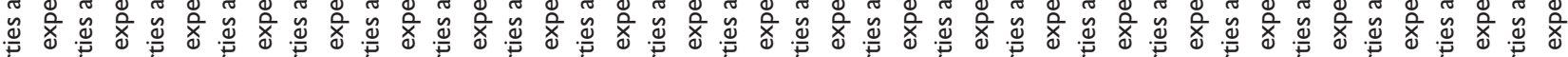

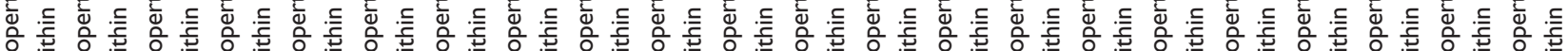

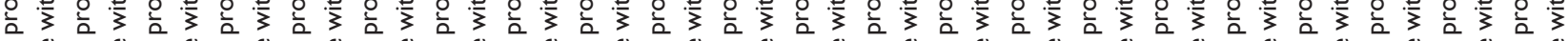

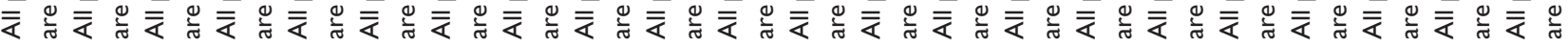

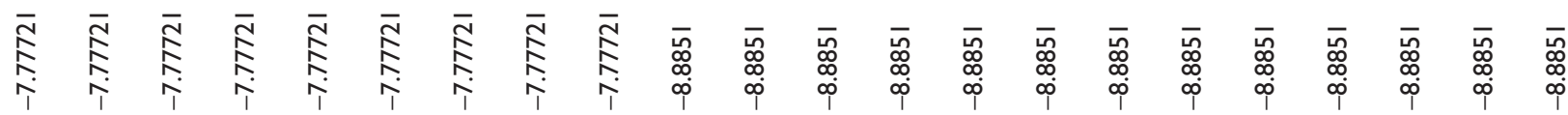

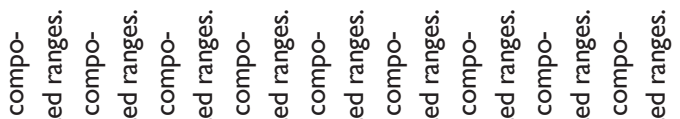

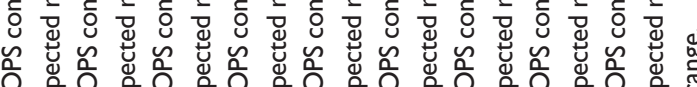

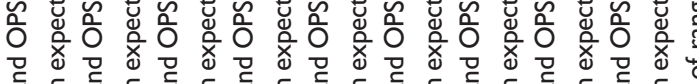

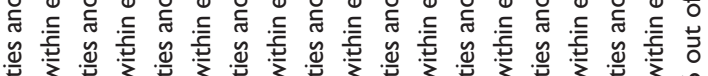

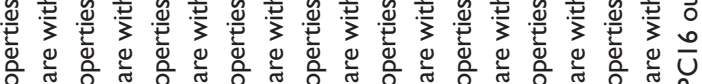

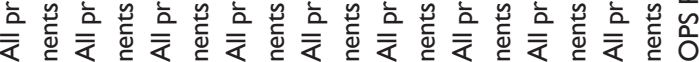

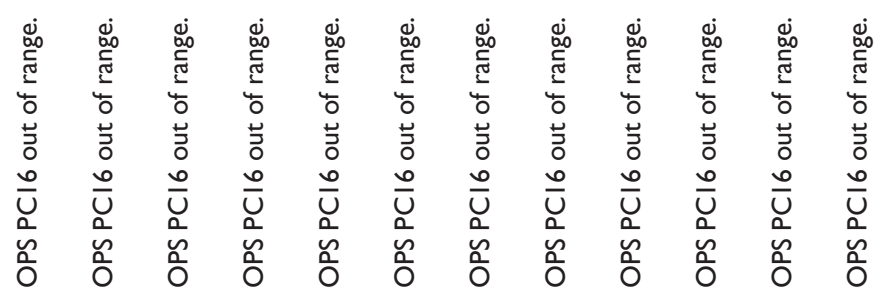

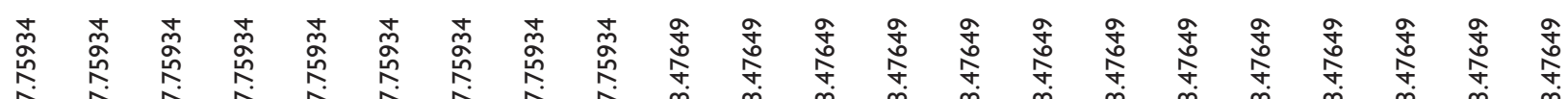

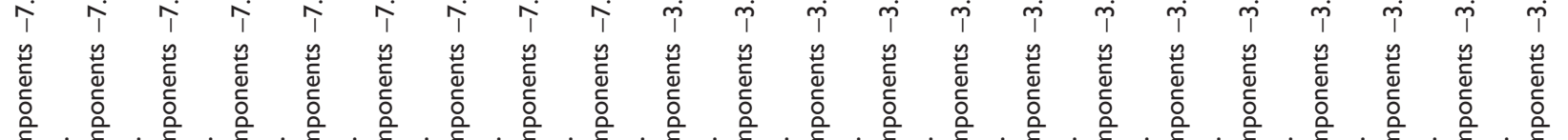

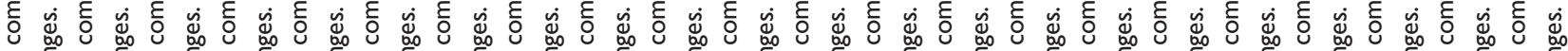
行 든

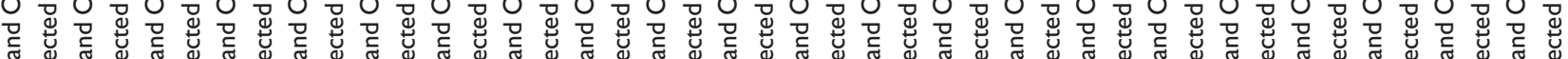
可

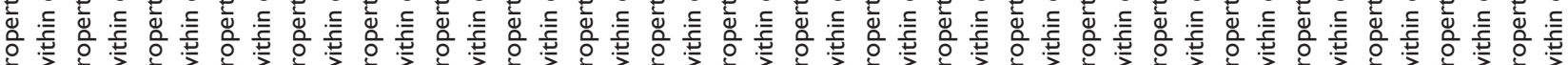

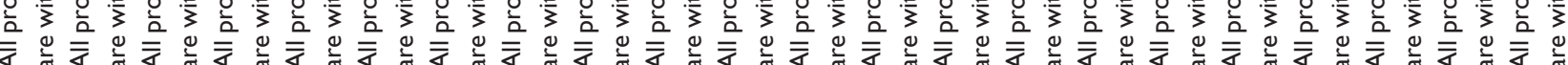

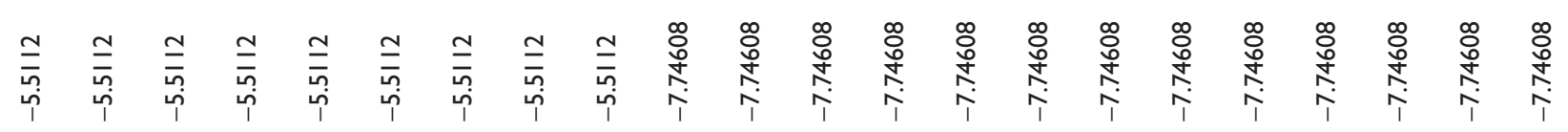$$
\text { œ ळ }
$$ 


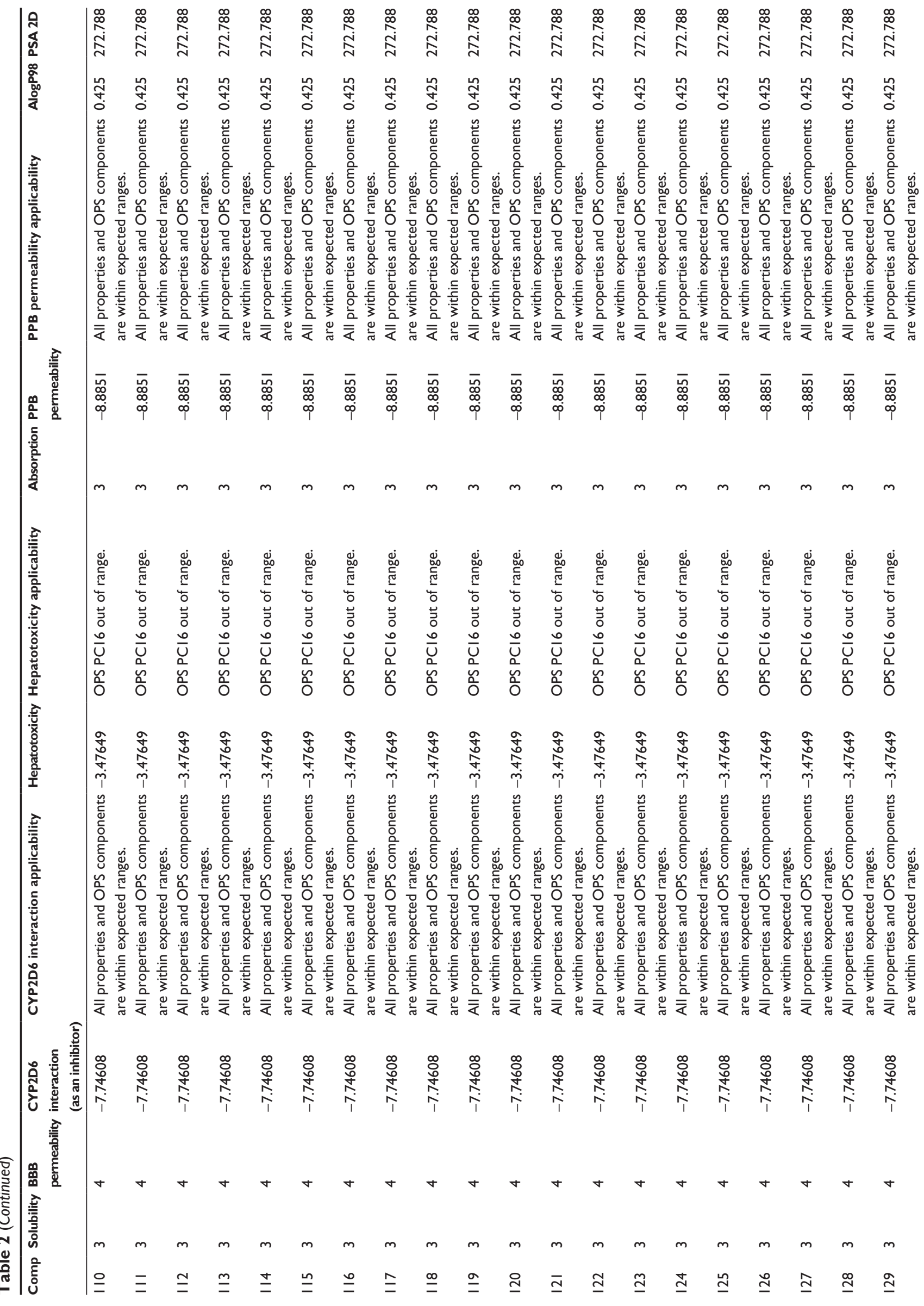




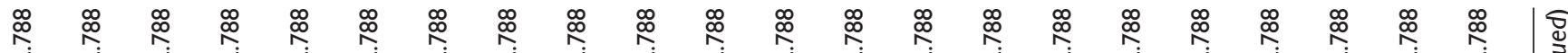

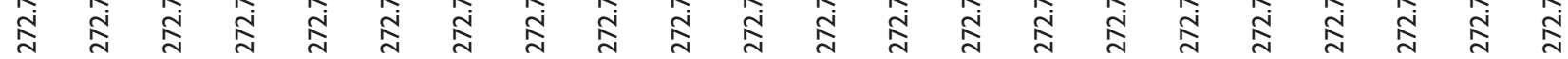

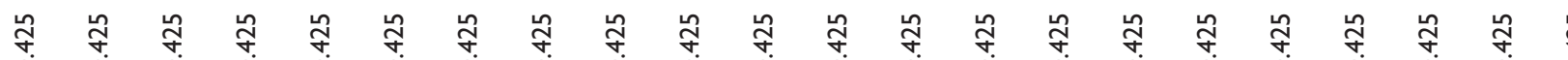

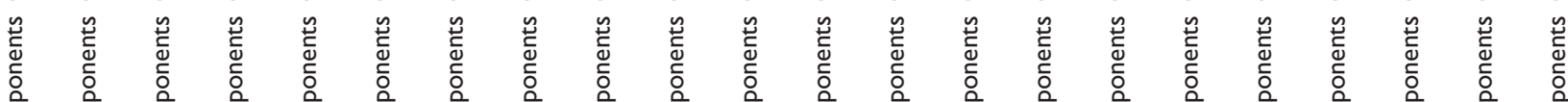

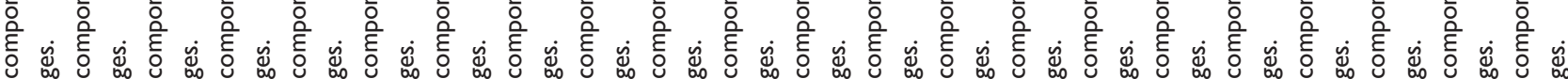

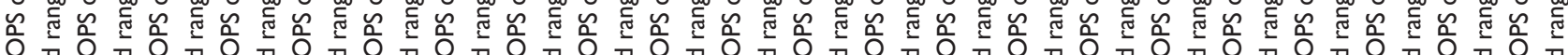

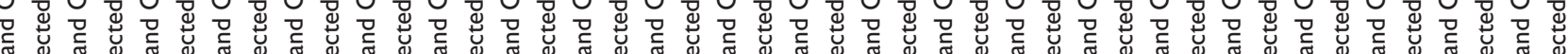

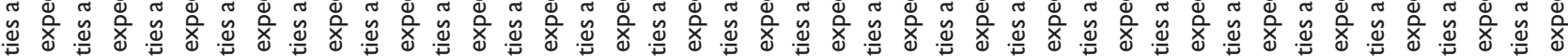

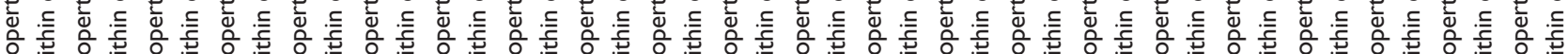

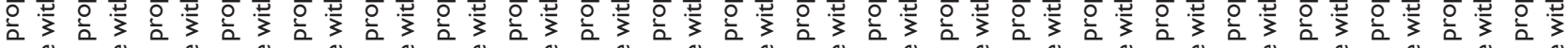

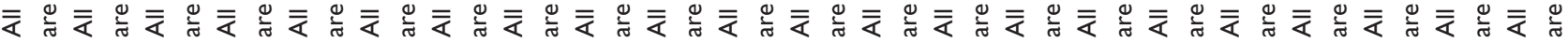

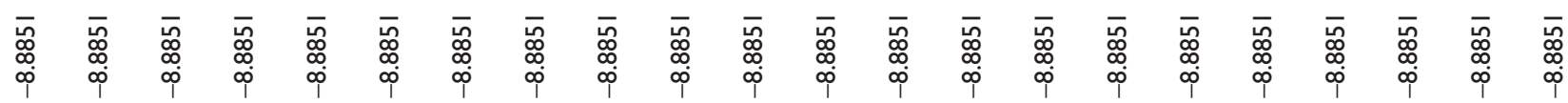

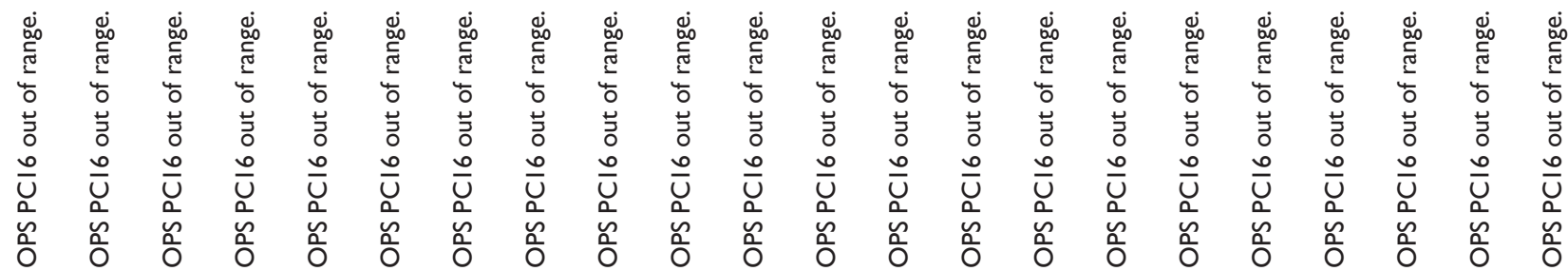

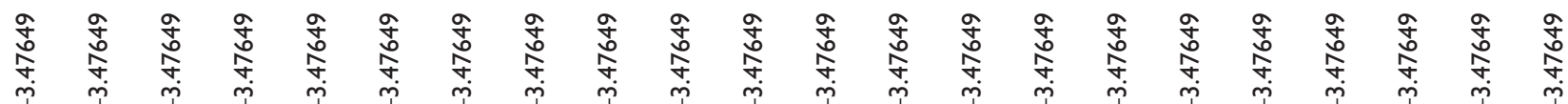

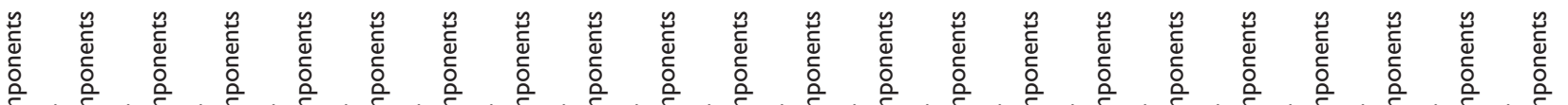

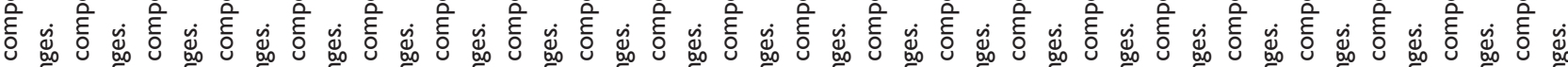

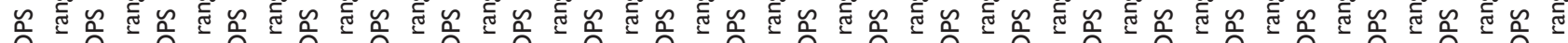

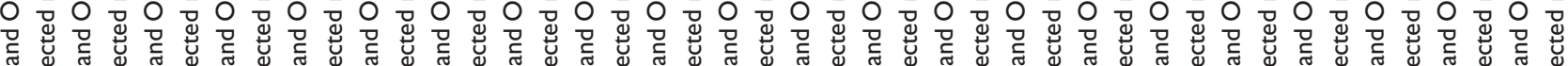

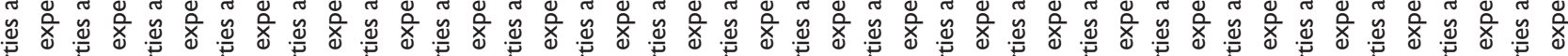

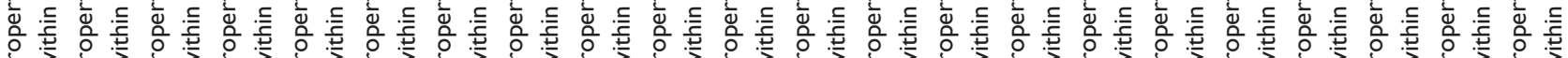

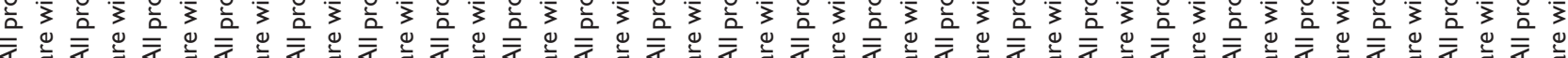

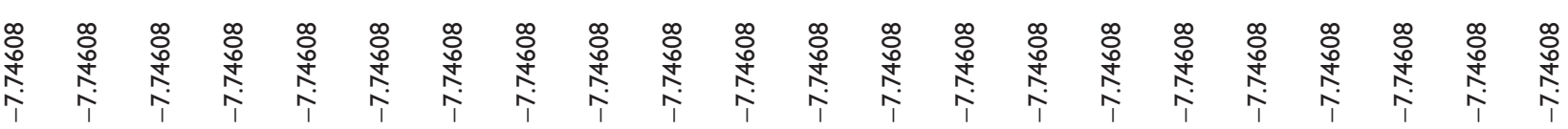

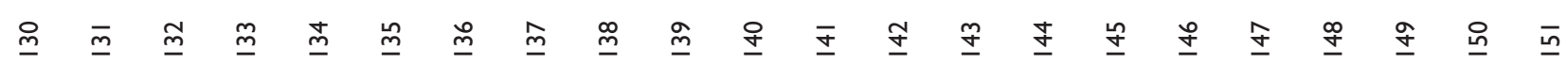




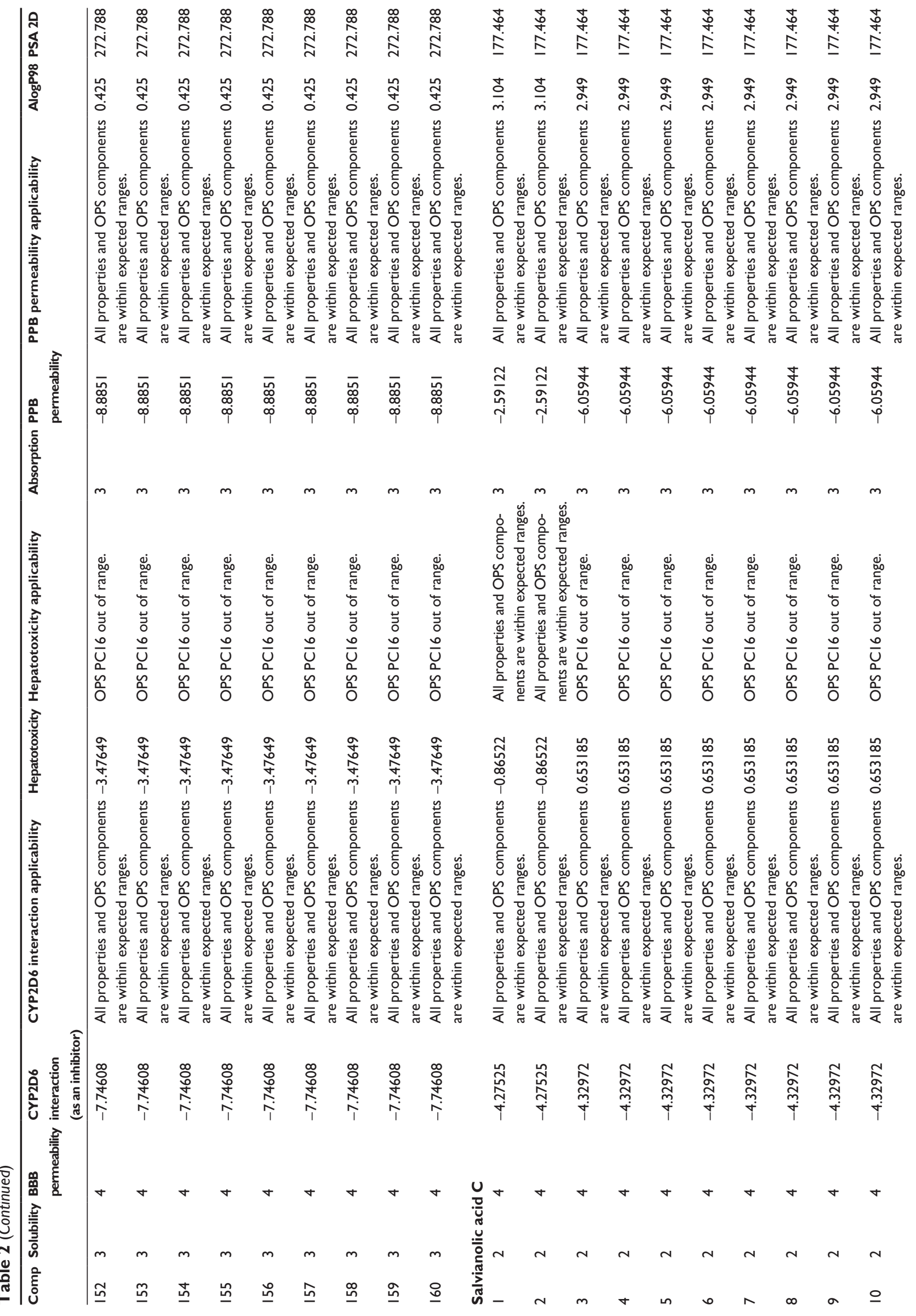




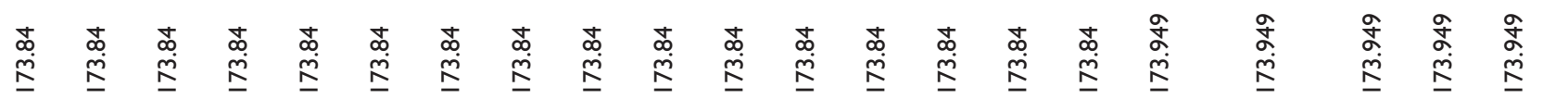

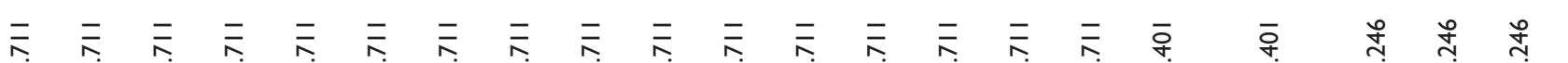

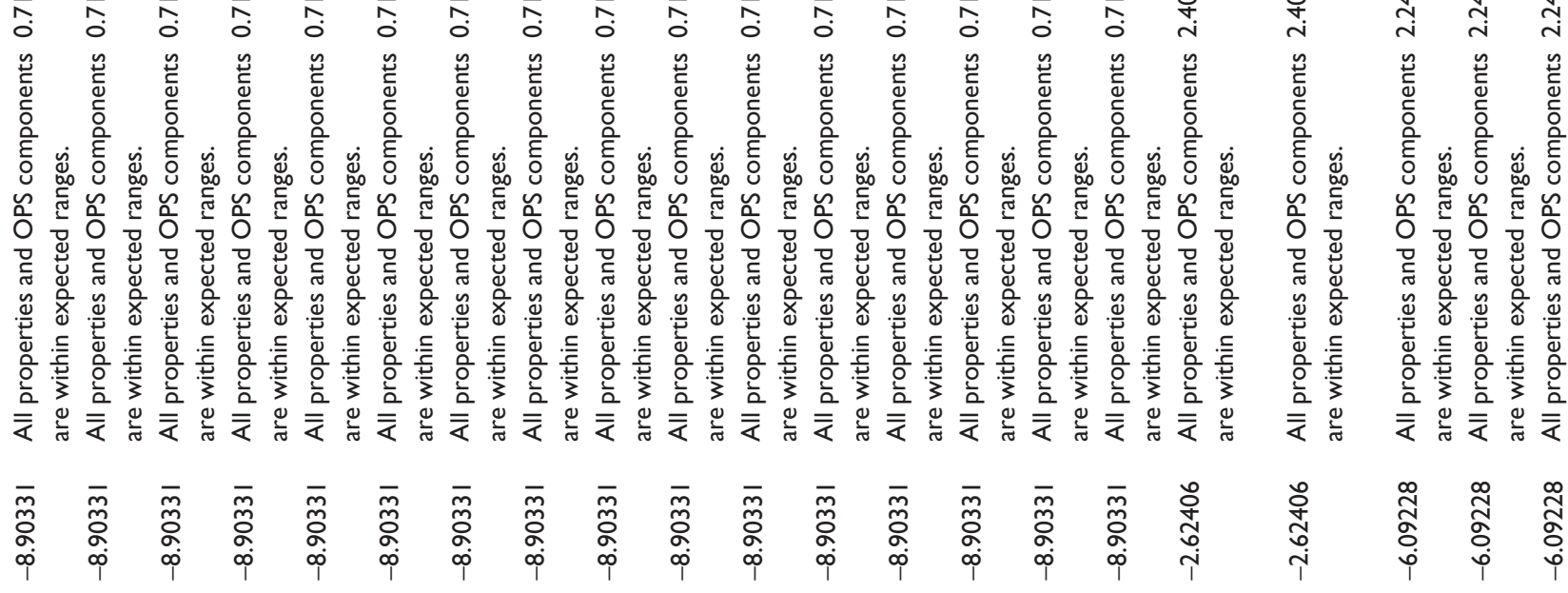

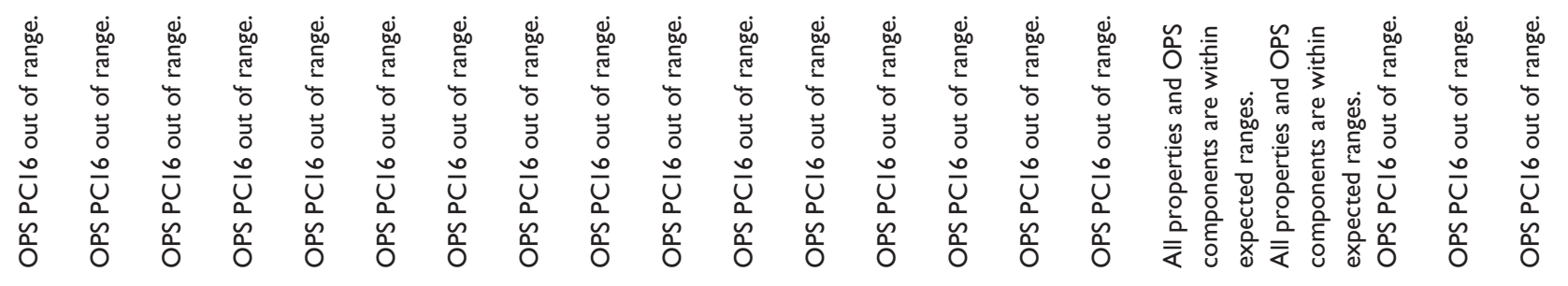

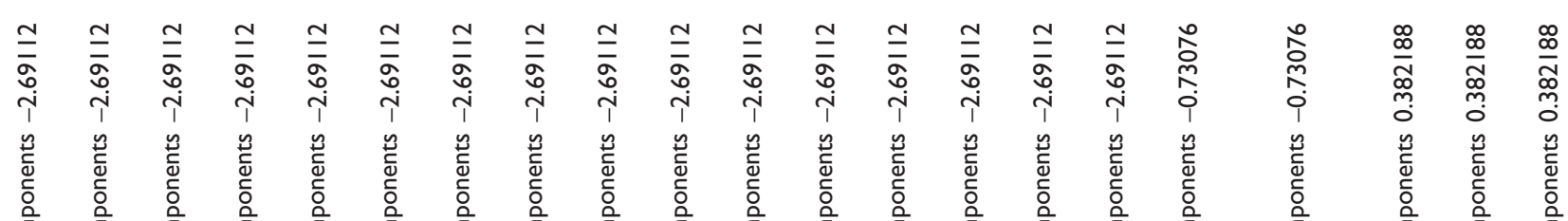

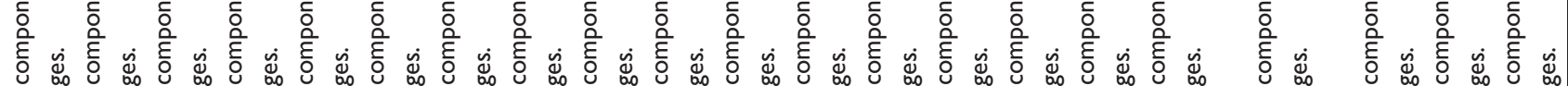

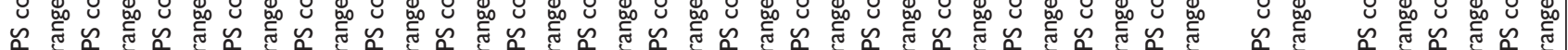

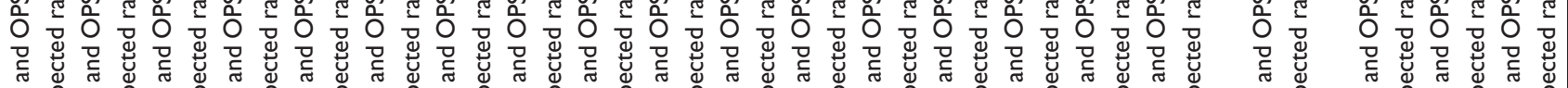

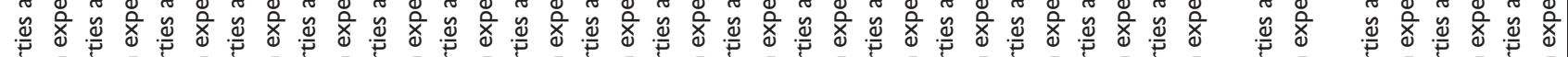

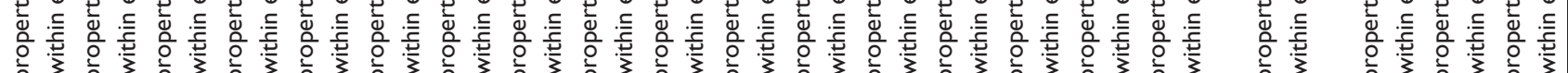

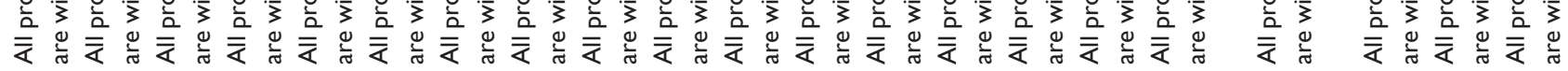

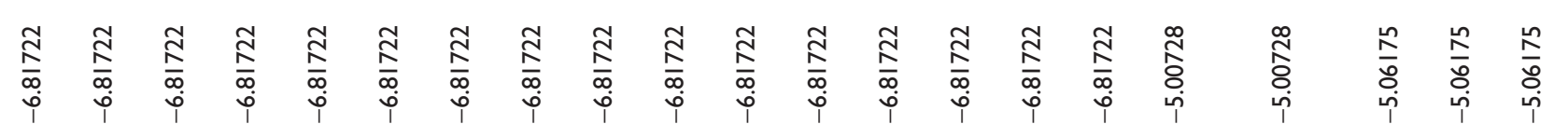

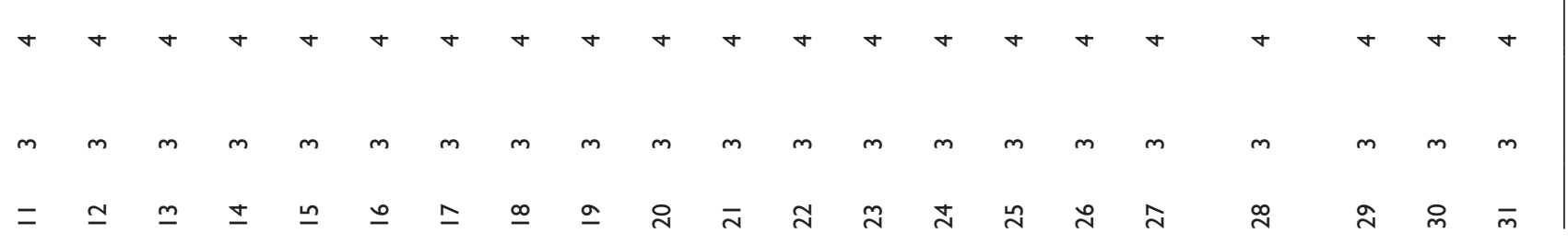




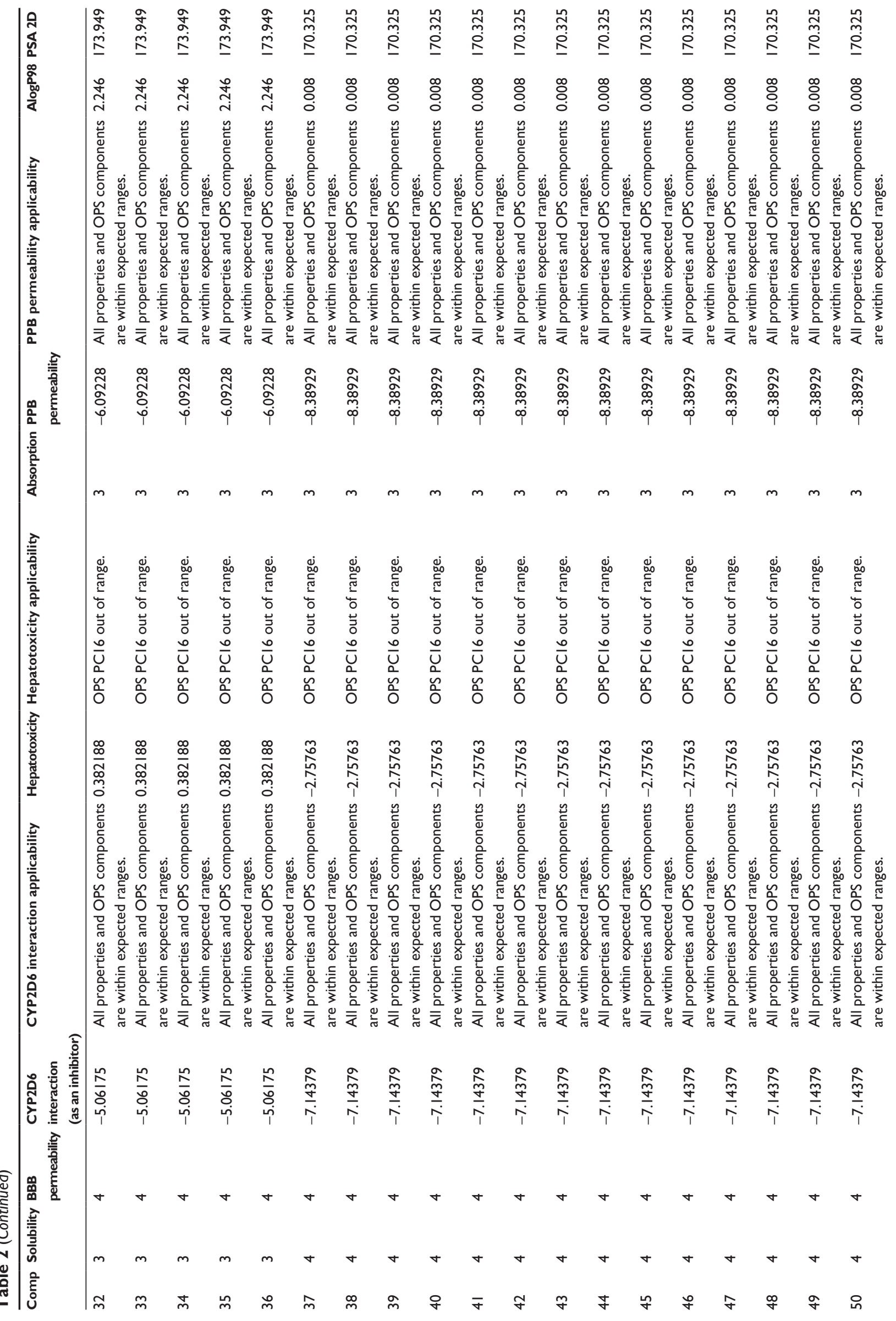




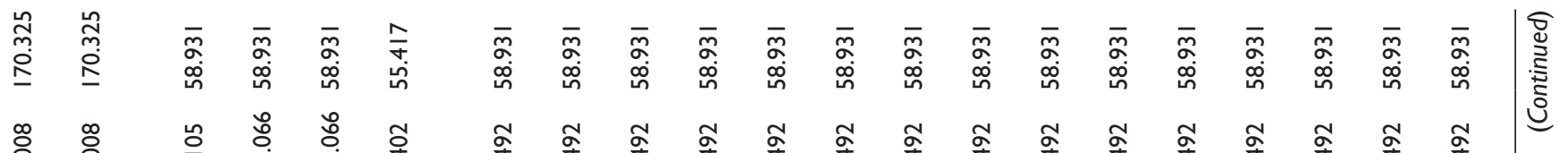

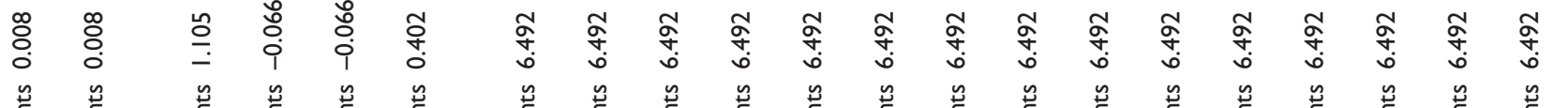

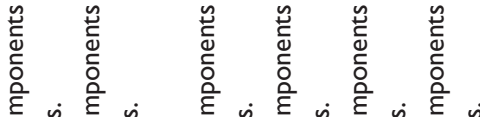

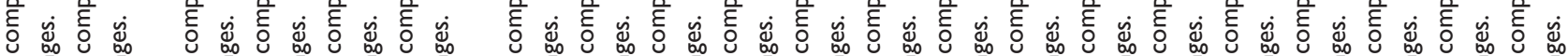

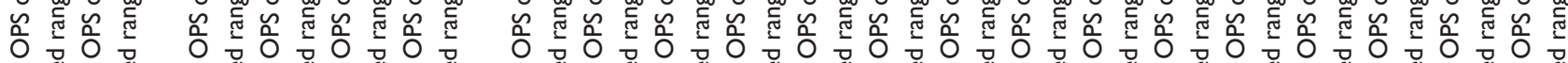

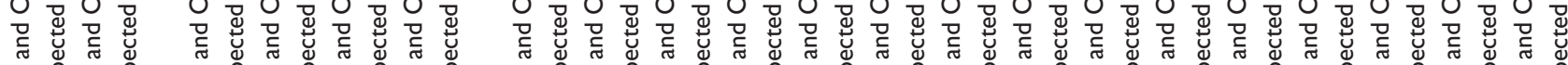

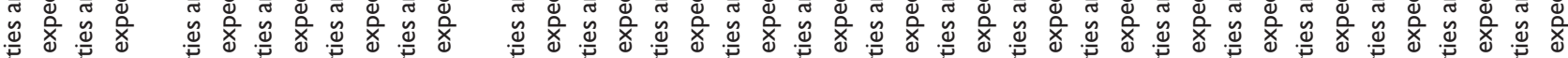

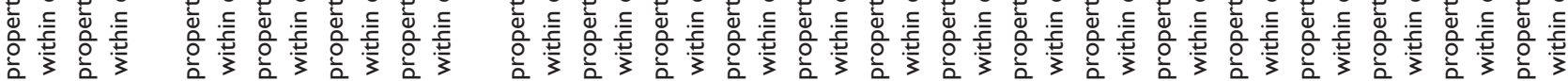

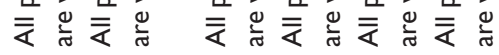

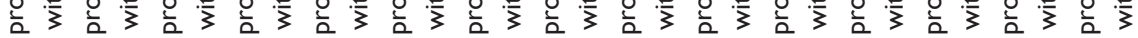

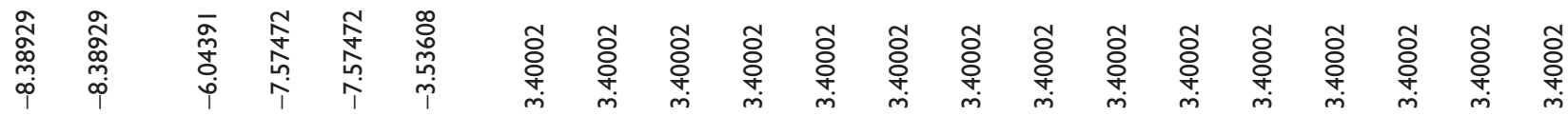

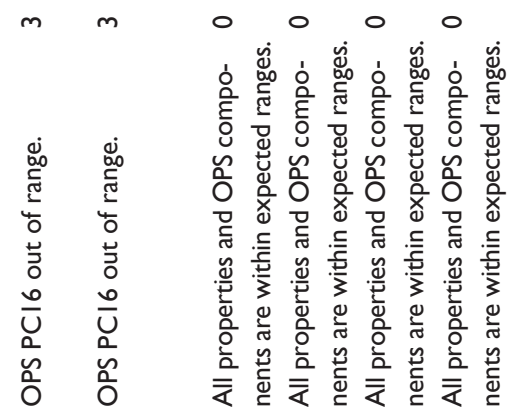

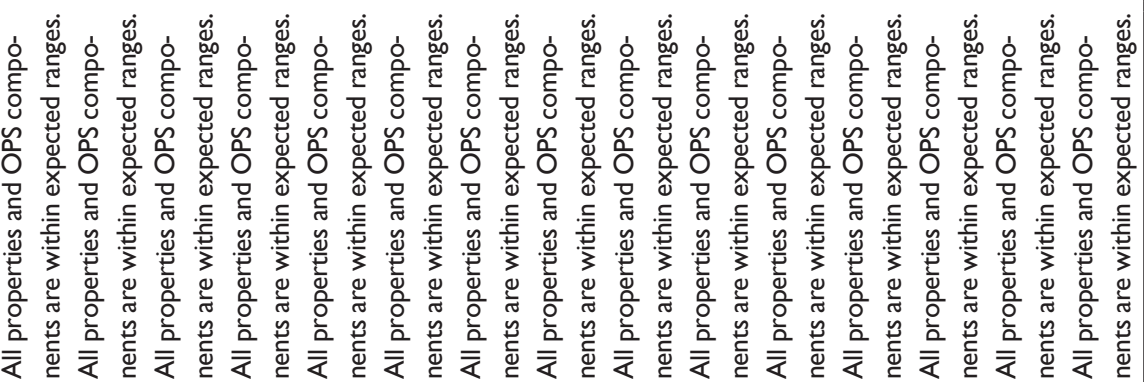

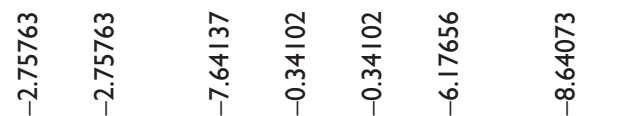

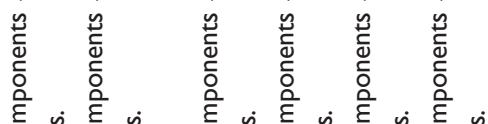

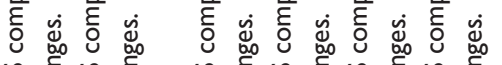

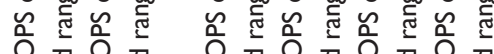

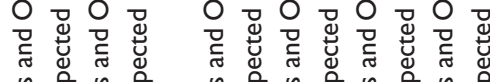

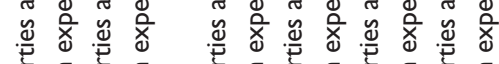

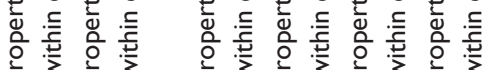

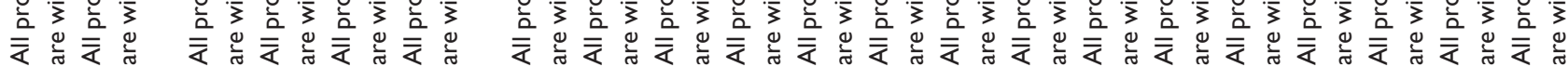

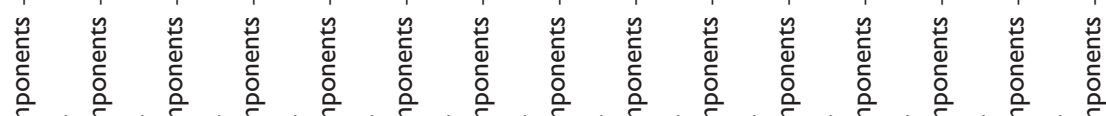

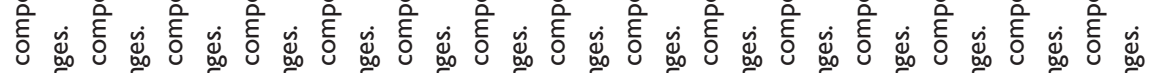

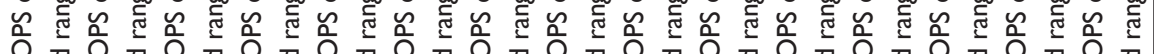

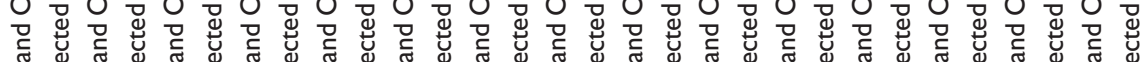

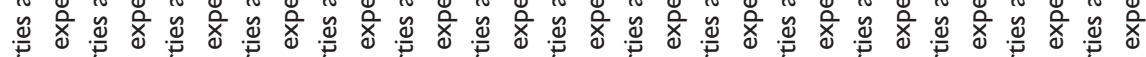

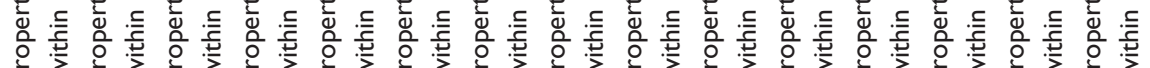

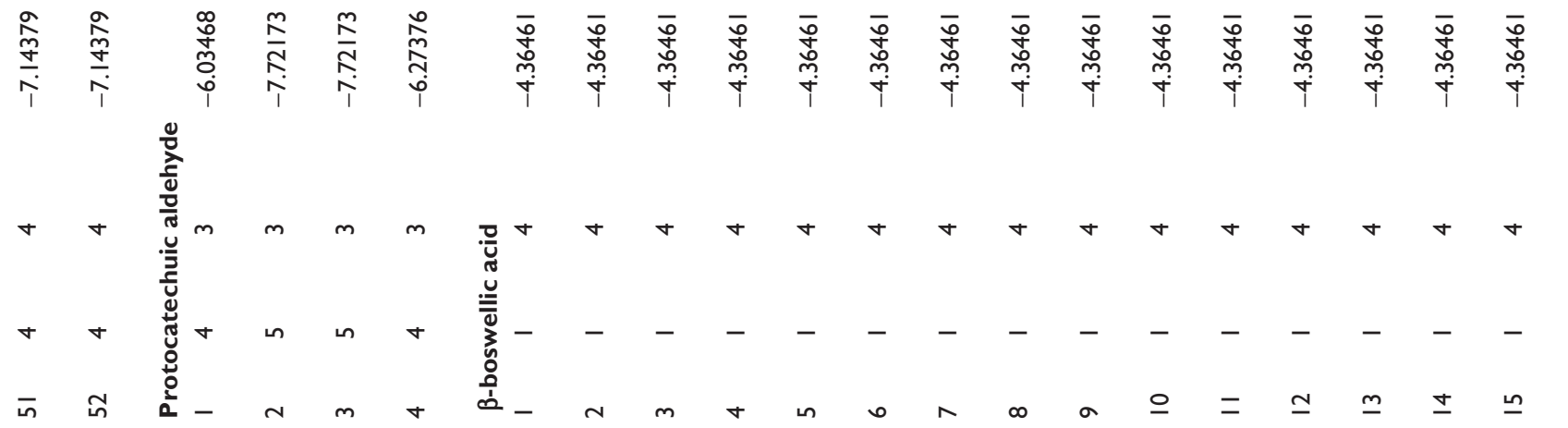




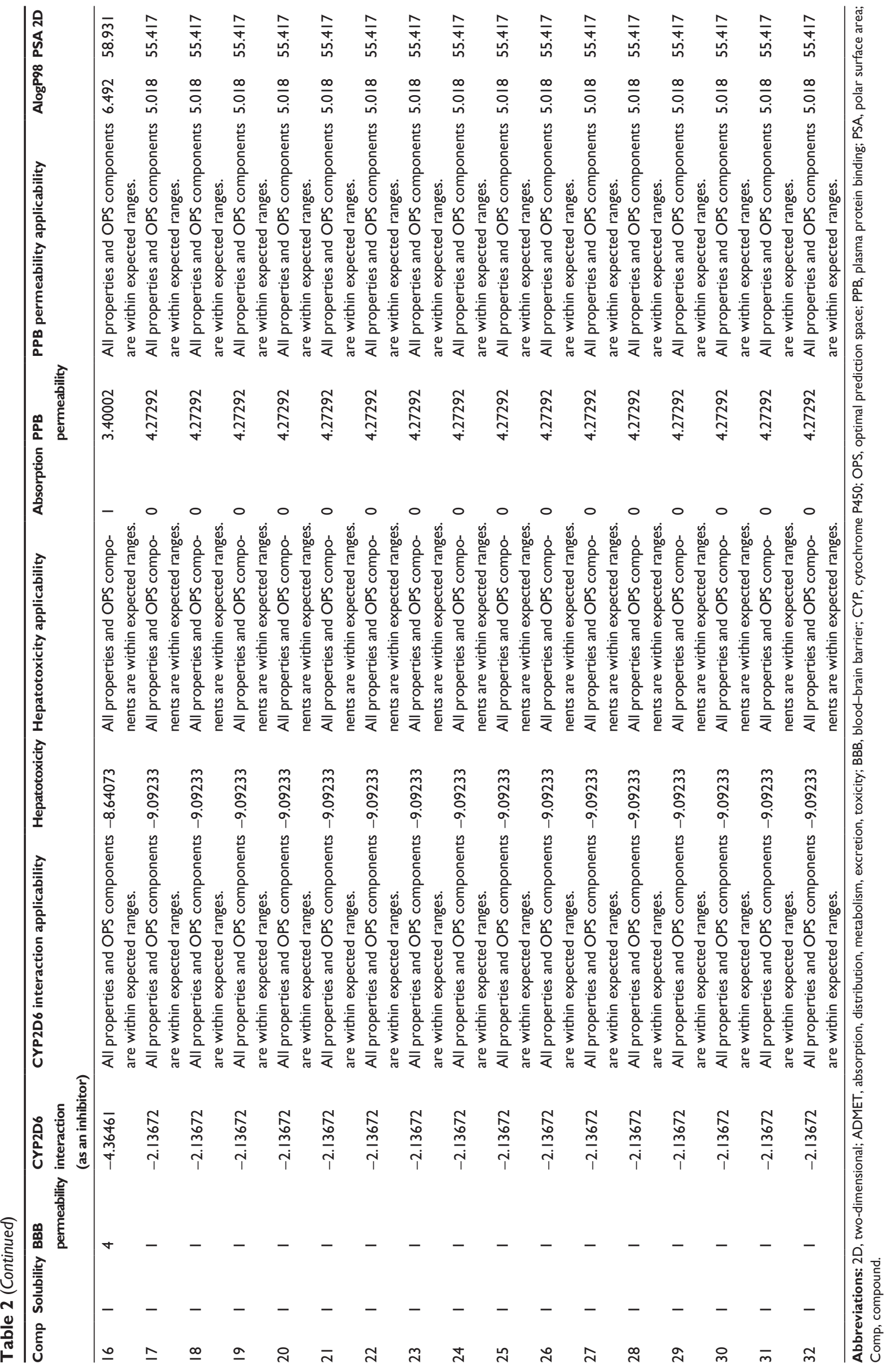


Table 3 Effect of HLXLD on MAP, LVSP, and LVEDP in different experimental groups of rats ( $\mathrm{n}=8$ per group) with coronary artery ligation-induced acute myocardial ischemia

\begin{tabular}{lllll}
\hline Groups & Dose & MAP $(\mathbf{m m H g})$ & LVSP $(\mathbf{m m H g})$ & LVEDP $(\mathbf{m m H g})$ \\
\hline Sham & - & $116.82 \pm 6.34$ & $95.17 \pm 14.99$ & $28.32 \pm 22.42$ \\
Model & - & $101.31 \pm 9.17^{*}$ & $77.77 \pm 18.94^{*}$ & $19.35 \pm 13.68$ \\
Positive control & $0.25 \mathrm{mg} / \mathrm{kg}$ & $107.61 \pm 10.72^{*}$ & $93.53 \pm 13.4 I^{*}$ & $20.13 \pm 8.07$ \\
(isosorbide mononitrate) & & & & \\
Low-dose HLXLD & $2.7 \mathrm{~g} / \mathrm{kg}$ & $107.75 \pm 7.48^{*}$ & $92.35 \pm 19.79$ & $18.92 \pm 9.07$ \\
Mid-dose HLXLD & $5.4 \mathrm{~g} / \mathrm{kg}$ & $109.19 \pm 9.86^{*}$ & $98.67 \pm 7.35^{*}$ & $26.72 \pm 12.63$ \\
High-dose HLXLD & $10.8 \mathrm{~g} / \mathrm{kg}$ & $110.78 \pm 8.05^{*}$ & $97.71 \pm 15.15^{*}$ & $20.59 \pm 10.68$ \\
\hline
\end{tabular}

Notes: The data are shown as the mean \pm standard deviation; ${ }^{*} P<0.05$ versus normal control; $* P<0.05$ versus model control.

Abbreviations: HLXLD, Huo Luo Xiao Ling Dan; MAP, mean arterial pressure; LVSP, left ventricular systolic pressure; LVEDP, left ventricular end-diastolic pressure.

marker for common injuries and disease. It is extensively expressed in body tissues, including in blood cells and heart muscle, and is released during tissue damage..$^{30}$ As shown in Table 5, the model group had a slight decrease in serum SOD levels and a mild increase in serum CK and LDH levels, but the difference was not statistically significant $(P>0.05$ by one-way ANOVA) when compared with the sham group. Furthermore, administration of HLXLD and isosorbide mononitrate did not have a significant effect on serum SOD, $\mathrm{CK}$, and LDH levels ( $P>0.05$ by one-way ANOVA) when compared with the model group.

\section{Effect of HLXLD on pathological changes in rats with coronary artery ligation- induced acute myocardial ischemia}

To further evaluate the therapeutic effect of HLXLD in rats with coronary artery ligation-induced acute myocardial ischemia, a pathological examination was performed. As shown in Figure 13, there were viable alterations in the morphology of the left ventricle. In the sham group, the cardiomyocytes were neatly arranged with no marked necrotic cell death or interstitial inflammatory cell infiltration (Figure 13). In the model group with coronary artery ligation-induced acute myocardial ischemia, there was marked necrosis of cardiomyocytes in multiple areas, and the necrotic cardiomyocytes underwent myoplasm dissolution and vacuolation (Figure 13). Furthermore, myocardial fibers were arranged in a disordered manner with a loose and disrupted structure, showing degeneration and breakage of the junctions between cardiomyocytes (Figure 13). Administration of HLXLD had an ameliorating effect on coronary artery ligation-induced acute myocardial ischemia, as indicated by the amelioration and restoration in the disrupted morphology of the left ventricle (Figure 13). Low-dose HLXLD $(2.7 \mathrm{~g} / \mathrm{kg})$ slightly improved the arrangement of myocardial fibers and reduced focal necrosis of cardiomyocytes. Administration of mid-dose $(5.4 \mathrm{~g} / \mathrm{kg})$ and high-dose $(10.8 \mathrm{~g} / \mathrm{kg})$ HLXLD had a markedly beneficial effect on coronary artery ligation-induced damage in the left ventricle. The arrangement of the myocardial fibers was restored in a wave-like pattern and the disrupted myoplasm was recovered (Figure 13). Moreover, in the positive group, rats administered isosorbide mononitrate $0.2 \mathrm{mg} / \mathrm{kg}$ showed amelioration of coronary artery ligation-induced tissue damage in the left ventricle (Figure 13). The arrangement of myocardial fibers was restored in a wave-like pattern and the disrupted myoplasm was recovered in the positive group (Figure 13). Taken together, the results indicate that HLXLD has a therapeutic effect on coronary artery ligation-induced tissue damage in the left ventricle in rats.

Table 4 Effect of HLXLD on HR and $\pm \mathrm{dp} / \mathrm{dt}_{\max }$ in different experimental groups of rats ( $\mathrm{n}=8$ per group) with coronary artery ligationinduced acute myocardial ischemia

\begin{tabular}{|c|c|c|c|c|}
\hline Groups & Dose & $\begin{array}{l}\text { HR } \\
\text { (beats/minute) }\end{array}$ & $\begin{array}{l}+ \text { dp/dt }_{\max } \\
\text { (mmHg/second) }\end{array}$ & $\begin{array}{l}-\mathrm{dp} / \mathrm{dt}_{\max } \\
\text { (mmHg/second) }\end{array}$ \\
\hline Sham & - & $453.37 \pm 16.24$ & $3,552.99 \pm 950.26$ & $3,428.9 I \pm I, 015.70$ \\
\hline Model & - & $419 \pm 19.3^{\#}$ & $2,703.8 \pm 1,239.9^{\#}$ & $2,570.36 \pm 1,046.04^{\#}$ \\
\hline $\begin{array}{l}\text { Positive control } \\
\text { (isosorbide mononitrate) }\end{array}$ & $0.25 \mathrm{mg} / \mathrm{kg}$ & $438.75 \pm|4.6|^{*}$ & $3,537.95 \pm 603.68^{*}$ & $3,222.20 \pm 699.59$ \\
\hline Low-dose HLXLD & $2.7 \mathrm{~g} / \mathrm{kg}$ & $439.75 \pm 12.47^{*}$ & $3,676.59 \pm 634.04 *$ & $3,378.62 \pm 627.80 *$ \\
\hline Mid-dose HLXLD & $5.4 \mathrm{~g} / \mathrm{kg}$ & $438 \pm 14.49 *$ & $3,888.93 \pm 465.52^{*}$ & $3,388.58 \pm 427.17^{*}$ \\
\hline High-dose HLXLD & $10.8 \mathrm{~g} / \mathrm{kg}$ & $436.75 \pm 14.38^{*}$ & $3,578.85 \pm 656.48^{*}$ & $3,274.23 \pm 683.88$ \\
\hline
\end{tabular}

Notes: The data are shown as the mean \pm standard deviation; $\# P<0.05$ versus normal control; $* P<0.05$ versus model control.

Abbreviations: HLXLD, Huo Luo Xiao Ling Dan; HR, heart rate; $\pm d p / d t_{\text {max }}$, maximum rate of left ventricular pressure rise and fall. 
Table 5 Effect of HLXLD on SOD, CK, and LDH levels in different experimental groups of rats ( $n=8$ per group) with coronary artery ligation-induced acute myocardial ischemia

\begin{tabular}{lllll}
\hline Groups & Dose & $\begin{array}{l}\text { SOD } \\
\text { (IU/L) }\end{array}$ & $\begin{array}{l}\text { CK } \\
\text { (IU/L) }\end{array}$ & LDH (IU/L) \\
\hline Sham & - & $0.56 \pm 0.021$ & $453 \pm 88$ & $4,968.36 \pm 238.63$ \\
Model & - & $0.55 \pm 0.024$ & $468 \pm 102$ & $5,126.35 \pm 356.16$ \\
$\begin{array}{l}\text { Positive control } \\
\text { (isosorbide }\end{array}$ & $0.25 \mathrm{mg} / \mathrm{kg}$ & $0.55 \pm 0.029$ & $45 \mathrm{I} \pm 108$ & $4,975.36 \pm 410.24$ \\
mononitrate) & & & & \\
Low-dose HLXLD & $2.7 \mathrm{~g} / \mathrm{kg}$ & $0.57 \pm 0.025$ & $466 \pm 89$ & $5,103.37 \pm 358.62$ \\
Mid-dose HLXLD & $5.4 \mathrm{~g} / \mathrm{kg}$ & $0.54 \pm 0.018$ & $449 \pm 89$ & $5,210.45 \pm 284.95$ \\
High-dose HLXLD & $10.8 \mathrm{~g} / \mathrm{kg}$ & $0.55 \pm 0.025$ & $470 \pm 132$ & $4,071.29 \pm 360.82$ \\
\hline
\end{tabular}

Note: The data are shown as the mean \pm standard deviation.

Abbreviations: HLXLD, Huo Luo Xiao Ling Dan; SOD, superoxide dismutase; CK, creatine kinase; LDH, lactate dehydrogenase.

\section{Effect of HLXLD on Bcl-2 and caspase 3 expression levels in rats with coronary} artery ligation-induced acute myocardial ischemia

Given the observed beneficial effects of HLXLD on coronary artery ligation-induced acute myocardial ischemia, we explored the molecular targets involved in this therapeutic effect. The molecular docking results showed potential interactions between the major active components of HLXLD and key regulators of apoptosis, so we tested the effect of HLXLD on the expression of caspase 3 and Bcl-2. As shown in Figure 14A and B, administration of HLXLD markedly altered the expression of key functional proteins, Bcl-2 and caspase 3 , in heart tissue. Bcl-2 is an antiapoptotic protein antagonizing the apoptosis process and preventing cells from apoptotic cell death. ${ }^{8}$ Caspase 3 is a key member of the caspase family and activation of caspase 3 is an indispensable step in mitochondria-dependent apoptosis. ${ }^{10}$ Furthermore, it has been proposed that caspase 3 is a promising therapeutic target in the treatment of heart failure. ${ }^{10}$ In comparison with the sham group, there was a $55.0 \%$ decline in expression of Bcl-2 ( $P<0.05$ by one-way ANOVA; Figure 14A and B), whereas there was a 2.9-fold increase in the level of cleaved caspase 3 in the model group (rats with coronary artery ligation-induced acute myocardial ischemia; $P<0.001$ by one-way ANOVA; Figure 14A and B). Administration of HLXLD reversed these changes in Bcl-2 and cleaved caspase 3 expression. Compared with the model group, there was a 1.3-fold, 1.4-fold, and 1.5-fold increase in expression of

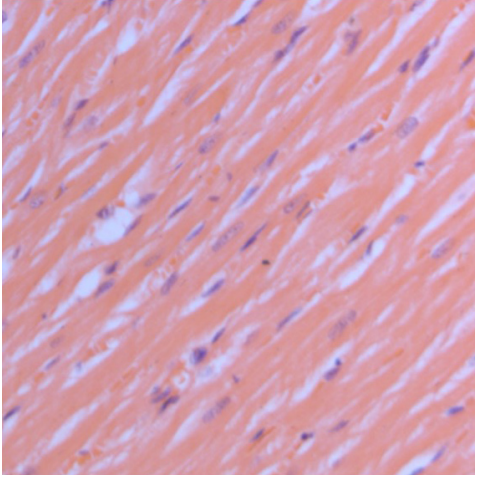

Sham

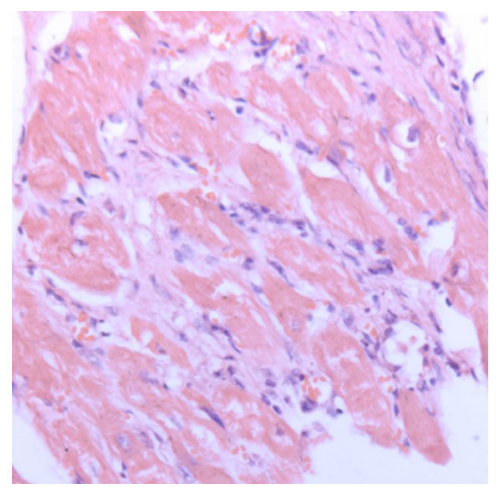

$2.7 \mathrm{~g} / \mathrm{kg} \mathrm{HLXLD}$

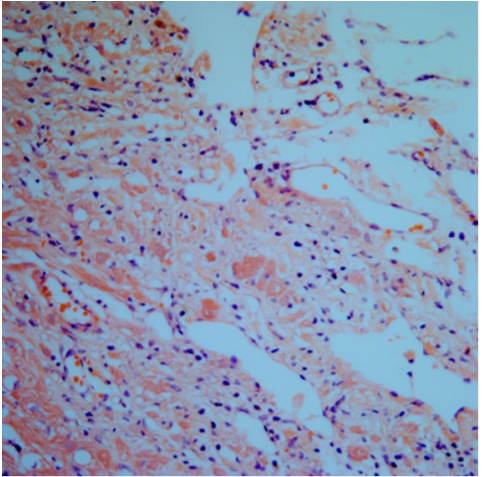

Model

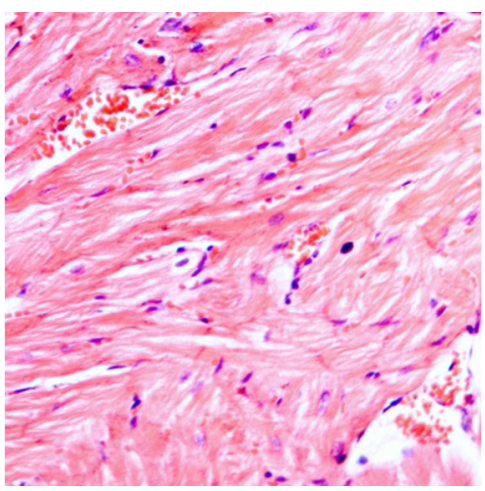

$5.4 \mathrm{~g} / \mathrm{kg} \mathrm{HLXLD}$

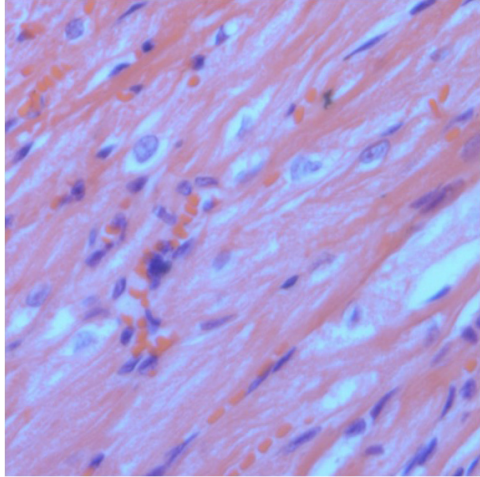

Positive control (isosorbide mononitrate)

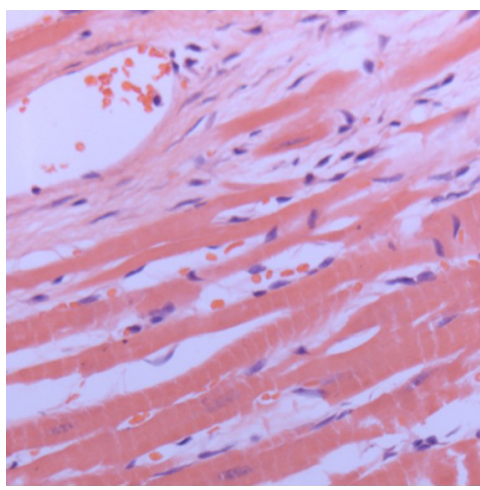

$10.8 \mathrm{~g} / \mathrm{kg} \mathrm{HLXLD}$

Figure 13 Morphological alterations in rat heart tissue.

Notes: Histological examination of rat left ventricle. The rats underwent sham operation or coronary artery ligation and were treated with saline, isosorbide mononitrate at $0.2 \mathrm{mg} / \mathrm{kg}$, or HLXLD at 2.7, 5.4, or $10.8 \mathrm{~g} / \mathrm{kg}$ for 4 weeks. Magnification $40 \times$.

Abbreviation: HLXLD, Huo Luo Xiao Ling Dan. 

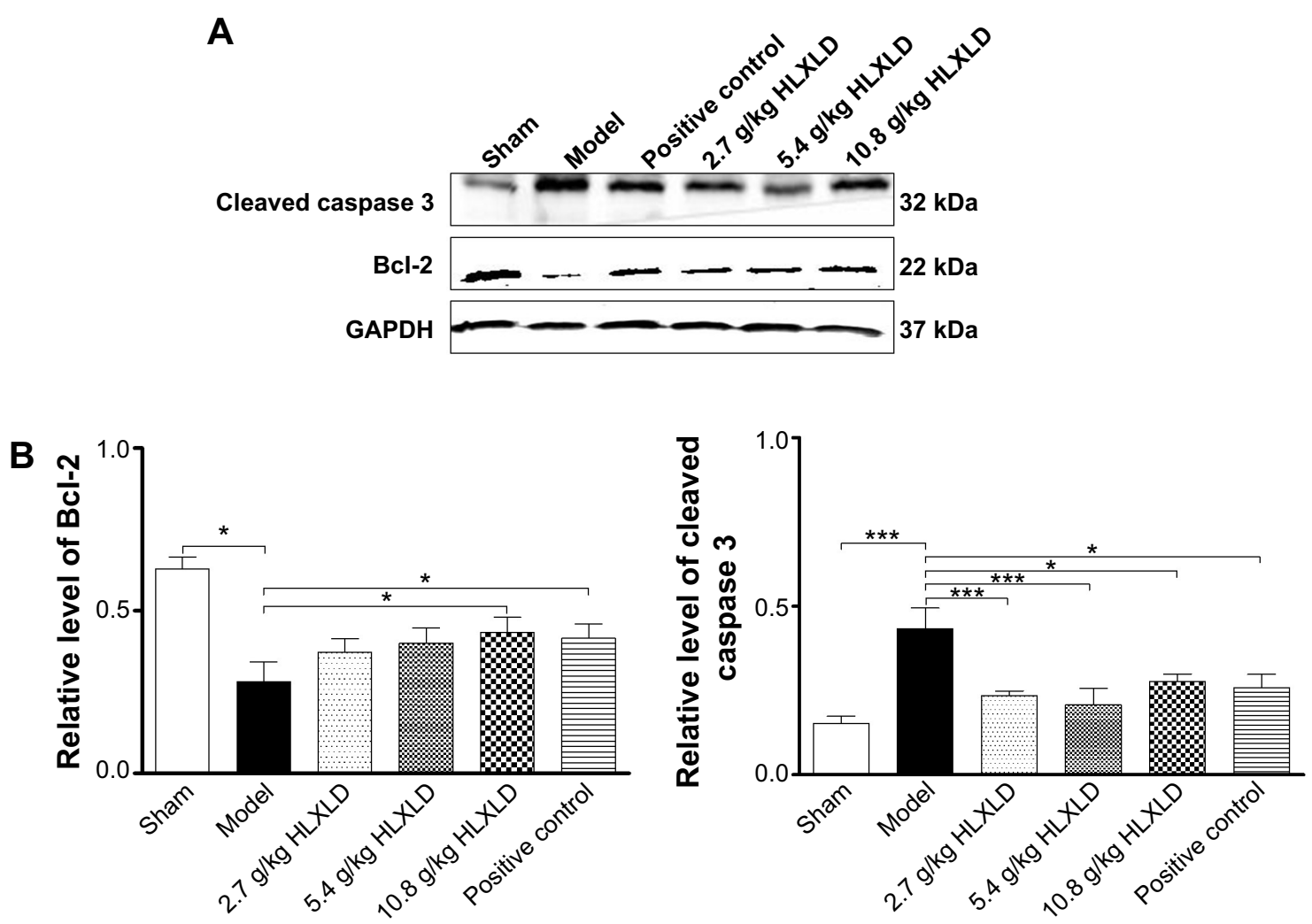

Figure 14 Huo Luo Xiao Ling Dan (HLXLD) upregulates the expression level of Bcl-2 but downregulates the expression of cleaved caspase 3 in the rat left ventricle. The rat left ventricle was collected and the protein samples were subjected to Western blotting assay.

Notes: (A) Representative blots of Bcl-2 and cleaved caspase 3. (B) Bar graphs showing the relative expression of Bcl-2 and cleaved caspase 3. GAPDH was used as the internal control. $* P<0.05, * * * P<0.001$ by one-way ANOVA.

Abbreviation: GAPDH, glyceraldehyde-3-phosphate dehydrogenase; ANOVA, analysis of variance; Bcl-2, B-cell lymphoma 2.

Bcl-2; but a $39.5 \%, 34.9 \%$, and $51.2 \%$ reduction in the level of cleaved caspase 3 when rats were administered HLXLD dosages of 2.7, 5.4, and $10.8 \mathrm{~g} / \mathrm{kg}$, respectively (Figure 14A and B). Moreover, there was a comparable therapeutic effect of high-dose HLXLD in the positive control group treated with $0.2 \mathrm{mg} / \mathrm{kg}$ isosorbide mononitrate. Taken together, HLXLD increases the expression level of Bcl-2 and decreases the level of activated caspase 3 in rats with coronary artery ligationinduced acute myocardial ischemia, which may contribute to the therapeutic effect of HLXLD in the treatment of IHD.

\section{Discussion}

IHD remains the most common cause of death worldwide and its exact pathogenesis is unknown, ${ }^{3}$ although the therapeutics currently available have greatly reduced the IHDrelated death rate. Increasing evidence shows that Chinese herbal medicines have beneficial effects in cardiovascular diseases. ${ }^{31}$ In the present study, we predicted the binding modes of ferulic acid, ligustilide, succinic acid, vanillic acid, tanshinone IIA, tanshinone IIB, danshensu, salvianolic acid A, salvianolic acid B, salvianolic acid C, protocatechuic aldehyde, and $\beta$-boswellic acid with human Bcl-xl,
Bak1, Bcl-2, procaspase 3, and caspase 9 using Discovery Studio ${ }^{\circledR}$ program 3.1, examined their effects, and investigated the possible molecular targets of HLXLD, a traditionally Chinese herbal formula, in a rat model of coronary artery ligation-induced acute myocardial ischemia. Our findings show that HLXLD had substantial therapeutic effects on acute myocardial ischemia in rats. HLXLD attenuated the coronary artery-induced tissue damage and ameliorated and restored the disrupted morphology of the left ventricle in the rats, with a significant increase in the expression of Bcl-2 and a decrease in the level of cleaved caspase 3.

The molecular docking study using Discovery Studio ${ }^{\circledR}$ program 3.1 showed that the major active components of HLXLD, including ferulic acid, ligustilide, succinic acid, vanillic acid, tanshinone IIA, tanshinone IIB, danshensu, salvianolic acid A, salvianolic acid $\mathrm{C}$, protocatechuic aldehyde, and $\beta$-boswellic acid can be easily docked into the active sites of human Bcl-xl, Bak1, Bcl-2, procaspase 3 , and caspase 9 via hydrogen bond formation, charge interaction, and/or $\pi-\pi$ interaction. Ferulic acid, ligustilide, succinic acid, vanillic acid, danshensu, salvianolic acid A, salvianolic acid $\mathrm{C}$, protocatechuic aldehyde, and $\beta$-boswellic acid interacted with human Bcl-xl, Bak1, Bcl-2, 
procaspase 3 , and/or caspase 9 mainly through hydrogen bond formation, and to a lesser extent via charge interaction and/or $\pi-\pi$ interaction, which may be due to the hydrophilic properties. In contrast, tanshinone IIA and tanshinone IIB bound to human Bcl-xl, Bak1, procaspase 3, and/or caspase 9 mainly via $\pi-\pi$ interaction, due to the hydrophobic properties. Indeed, the ADMET Predictor in Discovery Studio ${ }^{\circledR}$ program 3.1 predicted differential aqueous solubility of these components, which further explains the differential molecular interactions. In contrast, salvianolic acid B cannot be docked into the active sites of human Bcl-xl, Bak1, Bcl-2, procaspase 3, or caspase 9, due to violation of the "rule of five". Taken together, the molecular interactions between the major active components of HLXLD and the key regulators of apoptosis suggest a potential regulatory effect of HLXLD on apoptosis.

Myocardial ischemia is a clinical condition in which coronary blood flow is reduced by a variety of factors, such as accumulation of fatty deposits (atheroma) in the cells lining the wall of the coronary arteries. ${ }^{32,33}$ This results in a limited myocardial oxygen supply and decreased clearance of metabolites. ${ }^{32,33}$ In the clinic, myocardial ischemia is mainly related to a reduction of coronary blood flow. Any factors that reduce coronary perfusion pressure and/or increase coronary vascular resistance can cause insufficiency of the coronary artery blood supply and myocardial ischemia. ${ }^{32,33}$ When cardiac muscle undergoes ischemia and hypoxia, cardiac physiology and biochemistry will correspondingly change.

Under conditions of myocardial ischemia, regional myocardial contraction and relaxation is disrupted, as indicated by decreased myocardial contractility and cardiac output, a decrease in $\pm \mathrm{dp} / \mathrm{dt}_{\max }$, and an increase in LVEDP. ${ }^{34,35}$ MAP and $\pm \mathrm{dp} / \mathrm{dt}_{\max }$ reflect left ventricular and arterial systolic and diastolic function; ${ }^{32,36}$ of these, $\pm \mathrm{dp} / \mathrm{dt}_{\max }$ is a sensitive indicator of changes in myocardial diastolic parameters and function. ${ }^{26,30}$ Reduction of diastolic coronary pressure will lead to further aggravation of myocardial ischemia, leading to IHD. The most common cause of IHD is coronary atherosclerosis, and the left anterior descending coronary artery is most commonly involved vessel. Ligation of the left anterior descending coronary artery will cause a serious shortage of myocardial blood supply and a sharp decline in cardiac function, leading to a marked decrease in MAP, LVSP, heart rate, and $\pm \mathrm{dp} / \mathrm{dt}_{\max }{ }^{32,37}$ In the present study, we have demonstrated that administration of HLXLD mitigates the decrease in MAP, LVSP, heart rate, and $\pm \mathrm{dp} / \mathrm{dt}_{\max }$ in rats with acute myocardial ischemia. Our observations indicate that HLXLD has a beneficial effect on coronary artery ligation-induced dysfunction in the rat heart.
Furthermore, we examined the effect of administration of HLXLD on serum SOD, CK, and LDH, which are important indicators for clinical detection of the extent and scope of myocardial ischemia..$^{27,28,30} \mathrm{SOD}$ is an important radical scavenger in the human body, playing a role in the clearance of superoxide ions. ${ }^{27}$ Abnormalities in its activity and/ or expression have been implicated in the pathogenesis of many diseases, including those affecting the cardiovascular system. ${ }^{27}$ Under conditions of acute myocardial ischemia, SOD can protect myocardial cells from invasion by free oxygen radicals, maintain the integrity of the myocardial cell membrane, and reduce the damage caused by ischemia. ${ }^{27}$ $\mathrm{CK}$ and LDH are two enzymes present in myocardial cells, and act as biomarkers of myocardial infarction. ${ }^{28-30}$ Acute myocardial ischemia can cause hypoxic injury to a large number of myocardial cells, resulting in substantial release of CK and LDH into the blood. In our study, no significant difference was observed in these parameters, which may be because they were measured 4 weeks after coronary artery ligation was performed. However, pathological examination showed that administration of HLXLD markedly attenuated the ischemia-induced tissue damage, improved the arrangement of myocardial fibers, and reduced the focal necrosis of cardiomyocytes in rats. Overall, the results indicate that HLXLD has a therapeutic effect on IHD in rats.

Apoptosis is a fundamental biological phenomenon in cells, with an essential role in removing unwanted or abnormal cells in multicellular organisms. ${ }^{38,39}$ Apoptosis is not only a special type of cell death but also has great biological significance and involves complex molecular mechanisms. ${ }^{38,39}$ In particular, apoptosis is a key feature in the progression of heart disease and has an important role in adverse ventricular remodeling. ${ }^{6,40,41}$ Many factors can cause apoptosis, including a variety of ischemia, hypoxia, and toxicity factors, and the process of apoptosis involves a large number of proteins, including the caspase family. ${ }^{38,39,41}$ Caspases are a class of cysteine proteases that contain abundant cysteine at the active site and specifically catalyze hydrolysis of the aspartate portion in the substrate. ${ }^{42,43}$ Caspases are widely distributed in cells of various tissues, and their normal expression induces apoptosis. Caspase 3 is a key protease involved in apoptosis, acting as an executor and terminator of apoptosis, as well as a marker enzyme for occurrence of apoptosis. ${ }^{42,43}$ Furthermore, it has been proposed that caspase 3 may represent a promising therapeutic target in the treatment of cardiovascular disease. ${ }^{10}$ The Bcl-2 family is another group of proteins involved in apoptosis, members of which have antiapoptotic effects as well as proapoptotic effects. ${ }^{8}$ In different types of cells, Bcl-2 
can be located in the mitochondria, endoplasmic reticulum, and nuclear membrane, where it plays a role in antiapoptosis by preventing the release of mitochondrial cytochrome $\mathrm{c}^{8}$ In addition, Bcl-2 has a protective effect on cells, and its overexpression can reduce caspase activity. ${ }^{8}$ In the present study, our results show that administration of HLXLD markedly decreased the ischemia-induced activation of caspase 3 while increasing the expression of Bcl-2 in rats. These results indicate that HLXLD effectively inhibits apoptosis and protects myocardial cells against death, contributing to the therapeutic effect of HLXLD on coronary artery ligationinduced acute myocardial ischemia in rats.

Notably, there are a number of pharmacologically active components in HLXLD, such as tanshinones and salvianolic acids, which have been demonstrated to be protective cardiovascular components in vitro and in vivo. ${ }^{18,44,45}$ Their beneficial effects include improving the microcirculation, causing coronary vasodilatation, suppressing formation of thromboxane, and inhibiting platelet adhesion and aggregation, involving a number of important functional proteins and related signaling pathways, such as phosphatidylinositol-4, 5-bisphosphate 3-kinase/Akt signaling pathways. ${ }^{18,45}$ Our computational study showed that ferulic acid, ligustilide, succinic acid, vanillic acid, tanshinone IIA, tanshinone IIB, danshensu, salvianolic acid $\mathrm{A}$, salvianolic acid $\mathrm{C}$, protocatechuic aldehyde, and $\beta$-boswellic acid could interact with human Bcl-xl, Bak1, Bcl-2, procaspase 3, or caspase 9 via hydrogen bond formation, charge interaction, and/or $\pi-\pi$ interaction. Collectively, the results suggest that the therapeutic effect of HLXLD may be, at least in part, ascribed to the above-mentioned mechanisms of action of its bioactive components.

In summary, we have observed that the major active components of HLXLD interact with human Bcl-xl, Bak1, Bcl-2, procaspase 3 , or caspase 9 via hydrogen bond formation, charge interaction, and/or $\pi-\pi$ interaction using Discovery Studio ${ }^{\circledR}$ program 3.1. HLXLD mitigated the hemodynamic and serum enzyme changes induced by coronary artery ligation-induced acute myocardial ischemia, attenuated ischemia-induced tissue damage, and ameliorated myocardial function in rats. Furthermore, HLXLD inhibited apoptosis of cardiomyocytes by increasing the expression of Bcl-2 and decreasing the level of cleaved caspase 3 in rats with acute myocardial ischemia. Our findings suggest that HLXLD may be a promising agent for the treatment of IHD via targeting of apoptosis. More functional and mechanistic studies are warranted to elucidate the possible mechanisms involved, identify the therapeutic targets, and verify the efficacy and safety of HLXLD in the treatment of IHD.

\section{Acknowledgment}

This work was financially supported by the National Natural Science Foundation of China (project number: 81273657).

\section{Disclosure}

The authors report no conflicts of interest in this work.

\section{References}

1. Finegold JA, Asaria P, Francis DP. Mortality from ischaemic heart disease by country, region, and age: statistics from World Health Organisation and United Nations. Int J Cardiol. 2013;168(2):934-945.

2. Moran AE, Forouzanfar MH, Roth GA, et al. Temporal trends in ischemic heart disease mortality in 21 world regions, 1980 to 2010: the Global Burden of Disease 2010 study. Circulation. 2014;129(14): 1483-1492.

3. Nowbar AN, Howard JP, Finegold JA, Asaria P, Francis DP. 2014 global geographic analysis of mortality from ischaemic heart disease by country, age and income: statistics from World Health Organisation and United Nations. Int J Cardiol. 2014;174(2):293-298.

4. Lozano R, Naghavi M, Foreman K, et al. Global and regional mortality from 235 causes of death for 20 age groups in 1990 and 2010: a systematic analysis for the Global Burden of Disease Study 2010. Lancet. 2012;380(9859):2095-2128.

5. Zhang XH, Lu ZL, Liu L. Coronary heart disease in China. Heart. 2008; 94(9):1126-1131.

6. Abbate A, Narula J. Role of apoptosis in adverse ventricular remodeling. Heart Fail Clin. 2012;8(1):79-86.

7. Zhao Y, Xu Y, Zhang J, Ji T. Cardioprotective effect of carvedilol: inhibition of apoptosis in $\mathrm{H} 9 \mathrm{c} 2$ cardiomyocytes via the TLR4/NF-kB pathway following ischemia/reperfusion injury. Exp Ther Med. 2014;8(4): 1092-1096.

8. Czabotar PE, Lessene G, Strasser A, Adams JM. Control of apoptosis by the BCL-2 protein family: implications for physiology and therapy. Nat Rev Mol Cell Biol. 2014;15(1):49-63.

9. Shalini S, Dorstyn L, Dawar S, Kumar S. Old, new and emerging functions of caspases. Cell Death Differ. 2015 (in press).

10. Yang B, Ye D, Wang Y. Caspase-3 as a therapeutic target for heart failure. Expert Opin Ther Targets. 2013;17(3):255-263.

11. Zhang XC. Yi Xue Zhong Zhong Can Xi Lu (Records of Traditional Chinese Medicine with Reference to Western Medicine). Hebei Science and Technology Press, Shijiazhuang, Hebei, China, 1980 (in Chinese).

12. Nanjundaiah SM, Lee DY, Berman BM, Moudgil KD. Chinese herbal formula Huo-Luo-Xiao-Ling Dan protects against bone damage in adjuvant arthritis by modulating the mediators of bone remodeling. Evid Based Complement Alternat Med. 2013;2013:429606.

13. Nanjundaiah SM, Lee DY, Ma Z, et al. Modified Huo-Luo-Xiao-Ling Dan suppresses adjuvant arthritis by inhibiting chemokines and matrixdegrading enzymes. Evid Based Complement Alternat Med. 2012;2012: 589256.

14. Yang YH1, Rajaiah R, Lee DY, et al. Suppression of ongoing experimental arthritis by a Chinese herbal formula (Huo-Luo-Xiao-Ling Dan) involves changes in antigen-induced immunological and biochemical mediators of inflammation. Evid Based Complement Alternat Med.2011; 2011:642027.

15. Rajaiah R, Lee DY, Ma Z, et al. Huo-Luo-Xiao-Ling Dan modulates antigen-directed immune response in adjuvant-induced inflammation. J Ethnopharmacol. 2009;123(1):40-44.

16. Zhang RX, Fan AY, Zhou AN, et al. Extract of the Chinese herbal formula Huo Luo Xiao Ling Dan inhibited adjuvant arthritis in rats. J Ethnopharmacol. 2009;121(3):366-371.

17. Lao L, Fan AY, Zhang RX, et al. Anti-hyperalgesic and anti-inflammatory effects of the modified Chinese herbal formula Huo Luo Xiao Ling Dan (HLXL) in rats. Am J Chin Med. 2006;34(5):833-844. 
18. Cheng TO. Cardiovascular effects of Danshen. Int J Cardiol. 2007; 121(1):9-22.

19. Gao S, Liu Z, Li H, et al. Cardiovascular actions and therapeutic potential of tanshinone IIA. Atherosclerosis. 2012;220(1):3-10.

20. Wang ZX, Sun J, Howell CE, et al. Prediction of the likelihood of drug interactions with kinase inhibitors based on in vitro and computational studies. Fundam Clin Pharmacol. 2014;28(5):551-582.

21. Su J, Chang C, Xiang Q, et al. Xyloketal B, a marine compound, acts on a network of molecular proteins and regulates the activity and expression of rat cytochrome P450 3a: A bioinformatic and animal study. Drug Des Devel Ther. 2014;8:2555-2602.

22. Porrello ER, Mahmoud AI, Simpson E, et al. Transient regenerative potential of the neonatal mouse heart. Science. 2011;331(6020): 1078-1080.

23. Wang X, Wang XL, Chen HL, et al. Ghrelin inhibits doxorubicin cardiotoxicity by inhibiting excessive autophagy through AMPK and p38-MAPK. Biochem Pharmacol. 2014;88(3):334-350.

24. Cao X, Antonyuk SV, Seetharaman SV, et al. Structures of the G85R variant of SOD1 in familial amyotrophic lateral sclerosis. J Biol Chem. 2008;283(23):16169-16177.

25. Flynn JM, Melov S. SOD2 in mitochondrial dysfunction and neurodegeneration. Free Radic Biol Med. 2013;62:4-12.

26. Antonyuk SV, Strange RW, Marklund SL, Hasnain SS. The structure of human extracellular copper-zinc superoxide dismutase at $1.7 \mathrm{~A}$ resolution: insights into heparin and collagen binding. J Mol Biol. 2009; 388(2):310-326.

27. Fukai T, Ushio-Fukai M. Superoxide dismutases: role in redox signaling, vascular function, and diseases. Antioxid Redox Signal. 2011;15(6): 1583-1606.

28. Schlattner U, Tokarska-Schlattner M, Wallimann T. Mitochondrial creatine kinase in human health and disease. Biochim Biophys Acta. 2006; 1762(2):164-180.

29. Bong SM, Moon JH, Nam KH, Lee KS, Chi YM, Hwang KY. Structural studies of human brain-type creatine kinase complexed with the ADP- $\mathrm{Mg}^{2+}-\mathrm{NO}_{3}$ - -creatine transition-state analogue complex. FEBS Lett. 2008;582(28):3959-3965.

30. Kopel E, Kivity S, Morag-Koren N, Segev S, Sidi Y. Relation of serum lactate dehydrogenase to coronary artery disease. Am J Cardiol. 2012; 110(12):1717-1722.
31. Walden R, Tomlinson B. Cardiovascular disease. In: Benzie IFF, Wachtel-Galor S, editors. Herbal Medicine: Biomolecular and Clinical Aspects. 2nd ed. Boca Raton, FL, USA: CRC Press; 2011.

32. Libby P, Theroux P. Pathophysiology of coronary artery disease. Circulation. 2005;111(25):3481-3488.

33. Nabel EG, Braunwald E. A tale of coronary artery disease and myocardial infarction. N Engl J Med. 2012;366(1):54-63.

34. Pourmoghaddas M, Sanei H, Tavassoli A, Shojaei M. Estimation of left ventricular end diastolic pressure (LVEDP) in patients with ischemic heart disease by echocardiography and compare it with the results of cardiac catheterization. ARYA Atheroscler. 2011;7(1):1-6.

35. Mielniczuk LM, Lamas GA, Flaker GC, et al. Left ventricular enddiastolic pressure and risk of subsequent heart failure in patients following an acute myocardial infarction. Congest Heart Fail. 2007;13(4): 209-214.

36. Barton M. Prevention and endothelial therapy of coronary artery disease. Curr Opin Pharmacol. 2013;13(2):226-241.

37. Pant R, Marok R, Klein LW. Pathophysiology of coronary vascular remodeling: relationship with traditional risk factors for coronary artery disease. Cardiol Rev. 2014;22(1):13-16.

38. Jiang X, Wang X. Cytochrome C-mediated apoptosis. Annu Rev Biochem. 2004;73:87-106.

39. Estaquier J, Vallette F, Vayssiere JL, Mignotte B. The mitochondrial pathways of apoptosis. Adv Exp Med Biol. 2012;942:157-183.

40. Takemura G, Kanoh M, Minatoguchi S, Fujiwara H. Cardiomyocyte apoptosis in the failing heart - a critical review from definition and classification of cell death. Int J Cardiol. 2013;167(6):2373-2386.

41. Lee Y, Gustafsson AB. Role of apoptosis in cardiovascular disease. Apoptosis. 2009;14(4):536-548.

42. Li Y, Cohen R. Caspase inhibitors and myocardial apoptosis. Int Anesthesiol Clin. 2005;43(2):77-89.

43. Logue SE, Martin SJ. Caspase activation cascades in apoptosis. Biochem Soc Trans. 2008;36 Pt 1:1-9.

44. Ho JH, Hong CY. Salvianolic acids: small compounds with multiple mechanisms for cardiovascular protection. J Biomed Sci. 2011;18:30.

45. Zhou ZW, Xie XL, Zhou SF, Li CG. Mechanism of reversal of high glucose-induced endothelial nitric oxide synthase uncoupling by tanshinone IIA in human endothelial cell line EA.hy926. Eur J Pharmacol. 2012;697(1-3):97-105.
Drug Design, Development and Therapy

\section{Publish your work in this journal}

Drug Design, Development and Therapy is an international, peerreviewed open-access journal that spans the spectrum of drug design and development through to clinical applications. Clinical outcomes, patient safety, and programs for the development and effective, safe, and sustained use of medicines are a feature of the journal, which

\section{Dovepress}

has also been accepted for indexing on PubMed Central. The manuscript management system is completely online and includes a very quick and fair peer-review system, which is all easy to use. Visit http://www.dovepress.com/testimonials.php to read real quotes from published authors. 